\title{
Modern Surgical Treatment and
}

\section{Genomic Profiling of Pancreatic}

\section{Neuroendocrine Neoplasms}

- from the Operating Theater to the Gene Lab

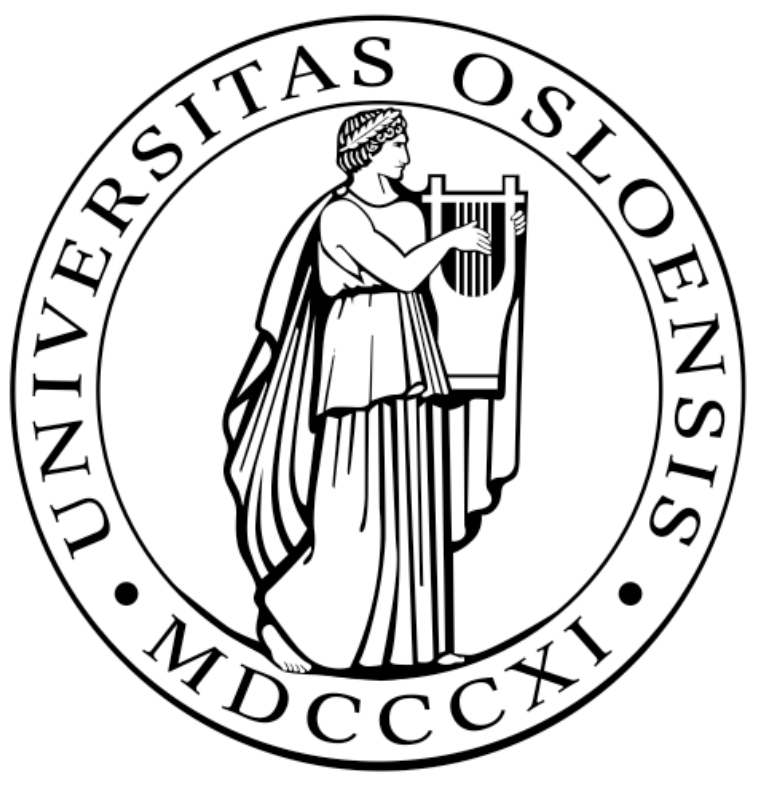

\section{Sven-Petter Haugvik}

Department of Hepato-Pancreato-Biliary Surgery

Oslo University Hospital, Oslo, Norway

Thesis submitted for the degree of philosophiae doctor $(\mathrm{PhD})$

May 2016

Faculty of Medicine, University of Oslo 
(C) Sven-Petter Haugvik, 2016

Series of dissertations submitted to the Faculty of Medicine, University of Oslo

ISBN 978-82-8333-318-3

All rights reserved. No part of this publication may be reproduced or transmitted, in any form or by any means, without permission.

Cover: Hanne Baadsgaard Utigard.

Print production: Reprosentralen, University of Oslo. 
This work is dedicated to CarciNor, the Norwegian patient advocacy association for neuroendocrine cancer, for its invaluable efforts in raising awareness about and support for patients and caregivers affected by neuroendocrine cancer. 



\section{Table of contents}

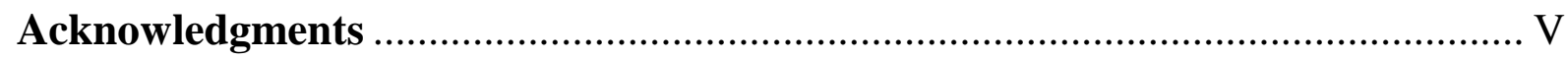

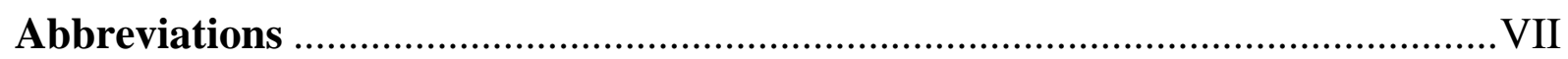

Publications included ....................................................................................... VII

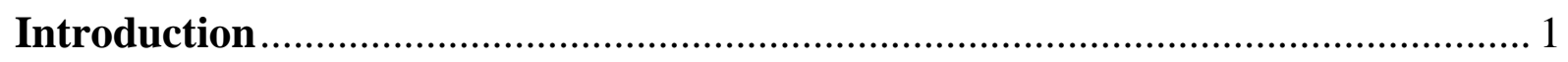

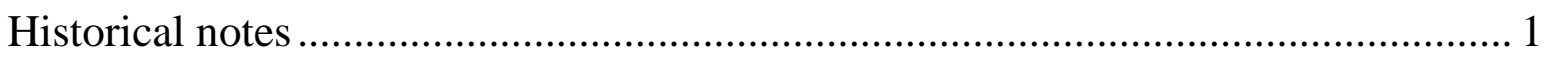

Pancreatic neuroendocrine neoplasms (PNENs) ................................................... 2

Definition, clinical presentation, and epidemiology .............................................. 2

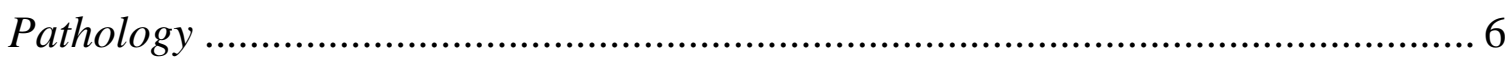

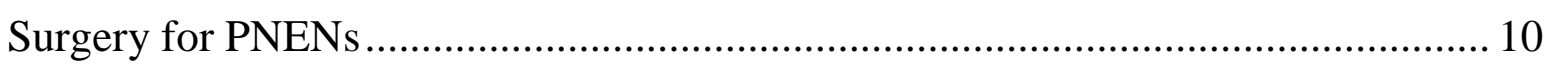

High-grade pancreatic neuroendocrine carcinoma (PNEC) ...................................... 14

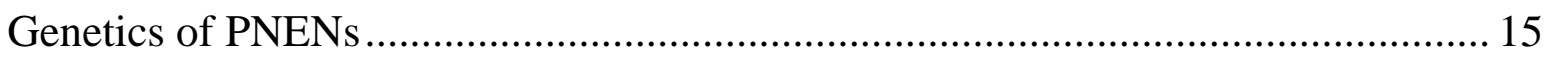

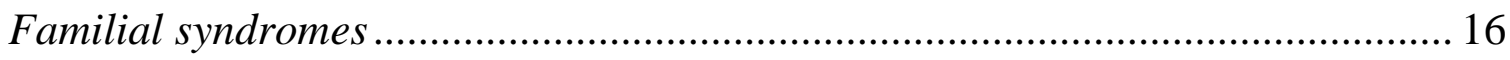

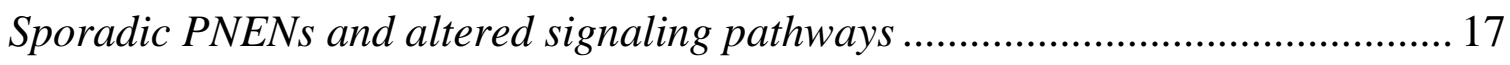

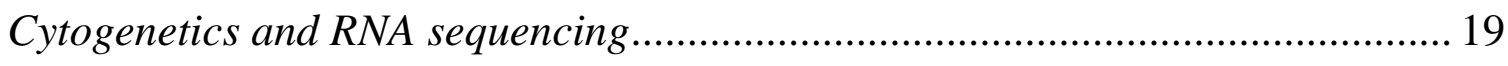

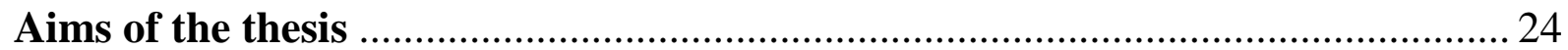

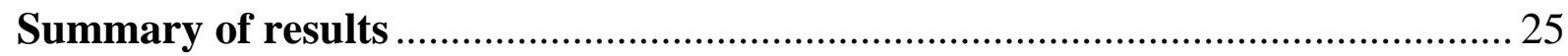

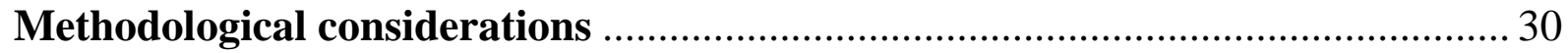

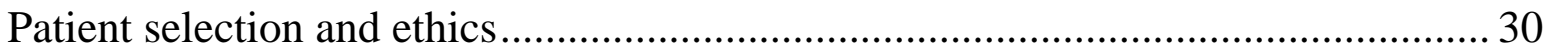

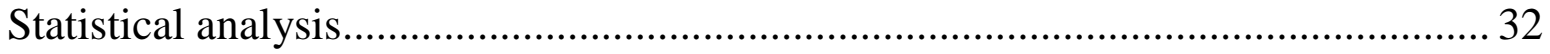

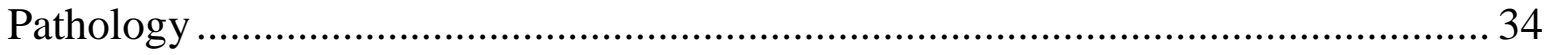

Karyotyping and comparative genomic hybridization (CGH) ................................. 36

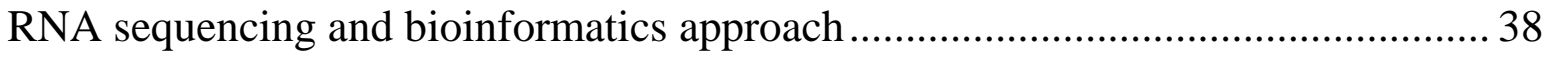

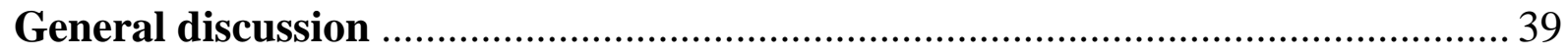

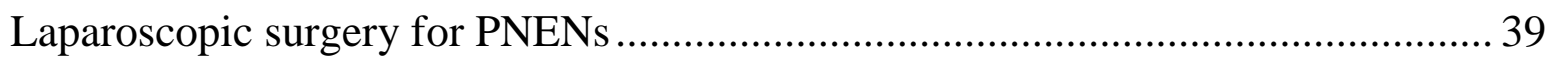

Vascular reconstruction in patients with PNENs ................................................... 46

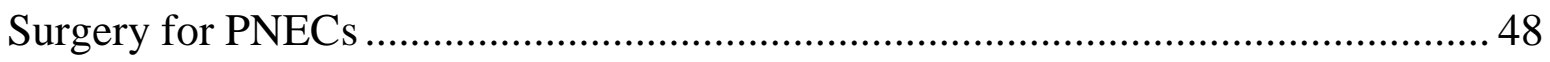

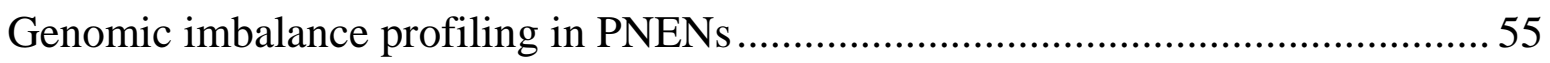

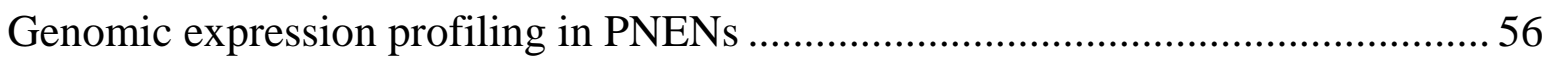

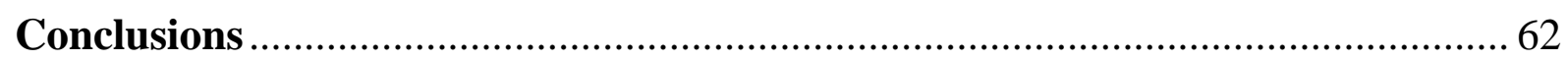

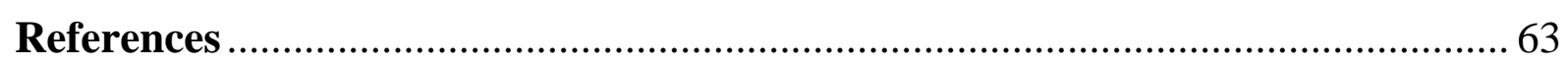

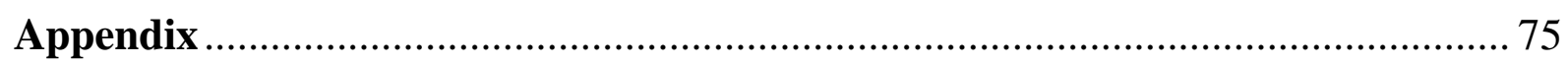

Surgical treatment algorithm for sporadic PNENs ................................................ 75

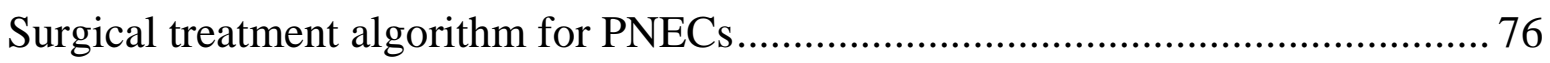

Papers I - V 



\section{Acknowledgments}

The work presented in this thesis was conducted from 2011 to 2016 at Oslo University Hospital. It is a result of close cooperation between the Department of Hepato-PancreatoBiliary Surgery at Oslo University Hospital and the following units: the Intervention Centre (Oslo University Hospital), the Section of Cancer Cytogenetics (Oslo University Hospital), and the Nordic Neuroendocrine Tumor Group. Financial support was granted by CarciNor, the Henrik Homan Foundation, and the Norwegian Society of Gastroenterology.

This thesis would not have been possible without the help, support, and guidance of many people. First, I want to address special thanks to my German colleagues, Prof. Merten Hommann and Dr. Daniel Kämmerer, for introducing me to the fascinating world of hepato-pancreato-biliary surgery and neuroendocrine oncology. As well-respected surgeons and close friends, you walked the first steps with me in both the clinical and research arena. It was a time that I will never forget.

I have always seen the importance of strong leadership and courage to make independent decisions, and want to thank Dr. Øystein Mathisen and Prof. Ivar Gladhaug for opening doors for me in Oslo. Thank you for believing in me and giving me freedom and responsibility to get the most out of my time as a $\mathrm{PhD}$ candidate. I would like to express my sincere gratitude to my main supervisor, Prof. Ivar Gladhaug, for your continuous support, patience, availability, and listening ear, also in times of frustration. I have been proud to be your PhD candidate. Prof. Bjørn Edwin has been an outstanding cosupervisor. With your exceptional ways of performing surgery, you have taught me the principal of "thinking out of the box" in the operating theater. Thanks for sharing your experience with me! Dr. Bård Røsok deserves my thanks for your lessons on discipline in surgical education - even though you have been a strict teacher. I admire your surgical skills, endurance and continuous work towards perfection. My sincere thanks also go to Dr. Knut Jørgen Labori for your genuine interest in my research and career path. I also want to thank my dear colleague Dr. Anne Waage for your positive attitude and personal encouragements. With your passion for adventure, you have also reminded me that there is a world outside the hospital. 
As a PhD candidate in a surgical department, I am glad that I also had the chance to get in touch with other disciplines. I would like to thank Prof. Sverre Heim and Dr. Francesca Micci, my other co-supervisors, for giving me access to their laboratory facilities and for continuously backing me up. Lisbeth Haugom, Anne Mette Eibak, and Ludmila Gorunova - thanks for your great support in the lab. Jim Thorsen, Daniel Vodák, and Eivind Hovig - thanks for helping me with RNA sequencing and data analysis. Prof. Halfdan Sørbye has inspired me to think big and deserves my thanks for exciting discussions on current developments in neuroendocrine oncology. I have also appreciated your sincere interest in surgery. I want to thank Thu Hong Thy Nguyen and Lisa Yuen Løvold for your great help in biobanking. Dr. Ragnhild Sørum Falk deserves many thanks for your great help in statistical analysis.

My special thanks go to my fellow mentees of the Pancreas 2000 program - Alastair Hayes, Darko Siuka, Per Hedenström, and Roberto Valente - and my mentors Gabriele Capurso and Björn Lindkvist. You have been such an inspiration to work with and have helped me keep focus on my research. Appreciation also goes to Dr. Mona-Elisabeth Revheim, Dr. Airazat Kazaryan, Dr. Espen Thiis-Evensen, Prof. Elin Kure, Dr. Bjørn Atle Bjørnbeth and Dr. Anders Bjørneboe for your encouragement.

Without the help of patients, colleagues, ward nurses, scrub nurses, and lab technicians, this thesis would not have been possible. Thanks to everyone for your help! The opponents of this thesis do also deserve my gratitude for their time.

There is a person who deserves more thanks and respect than any other person. Her name is Severina and she is my wife. Without you, nothing of this would have come true. Thanks for being a wife of noble character! I also want to thank my parents for all their support and for having led me with good example from my early days. Above all, I want to thank God who gave me life. I thank you for the opportunity you have given me to explore the wonders of your creation.

Oslo, April 2016

Sven-Petter Haugvik 


\section{Abbreviations}

AJCC - American Joint Cancer Committee ANCA - average number of copy aberrations

CGH - comparative genomic hybridization CI - confidence interval

ENETS - European Neuroendocrine

Tumor Society

FC - fold change

GEP-NEC - gastro-entero-pancreatic neuroendocrine carcinoma

GEP-NEN - gastro-entero-pancreatic neuroendocrine neoplasm

HR - hazard ratio

Ki67 - antigen Ki67 encoded by the MKI67 gene

LDP - laparoscopic distal pancreatectomy

LE - laparoscopic enucleation

MEN-1 syndrome - multiple endocrine neoplasia type 1 syndrome mTOR - mammalian target of rapamycin NF-1 - neurofibromatosis type 1

NNTG - Nordic Neuroendocrine Tumor

Group

PCR - polymerase chain reaction

PDAC - pancreatic ductal adenocarcinoma
PNEC - high-grade pancreatic neuroendocrine carcinoma, G3

NANETS - North American

Neuroendocrine Tumor Society

NEC - neuroendocrine carcinoma

NEN - neuroendocrine neoplasm

NET - neuroendocrine tumor

PNEN - pancreatic neuroendocrine neoplasm

PNET - pancreatic neuroendocrine tumor, G1 and G2

POPF - postoperative pancreatic fistula PS - performance status

RAMPS - radical antegrade modular pancreatosplenectomy

RNA-seq - RNA sequencing

SEER - Surveillance, Epidemiology, and End Results Program

TNM - tumor-node-metastasis

TSC - tuberous sclerosis complex

UICC - International Union for Cancer

Control

VHL syndrome - von Hippel-Lindau syndrome

WHO - World Health Organization 


\section{Publications included}

I. Haugvik SP, Marangos IP, Røsok BI, Pomianowska E, Gladhaug IP, Mathisen O, Edwin B.

Long-term outcome of laparoscopic surgery for pancreatic neuroendocrine tumors.

World J Surg. 2013 Mar; 37(3):582-90.

II. Haugvik SP, Labori KJ, Waage A, Line PD, Mathisen Ø, Gladhaug IP. Pancreatic surgery with vascular reconstruction in patients with locally advanced pancreatic neuroendocrine tumors. J Gastrointest Surg. 2013 Jul; 17(7):1224-32.

III. Haugvik SP, Janson ET, Österlund P, Langer SW, Falk RS, Labori KJ, Vestermark LW, Grønbæk H, Gladhaug IP, Sorbye H.

Surgical treatment as a principle for patients with high-grade pancreatic neuroendocrine carcinoma: a Nordic multicenter comparative study. Ann Surg Oncol. 2016 May; 23(5):1721-8.

IV. Haugvik SP, Gorunova L, Haugom L, Eibak AM, Gladhaug IP, Heim S, Micci F.

Loss of 11p11 is a frequent and early event in sporadic nonfunctioning pancreatic neuroendocrine neoplasms. Oncol Rep. 2014 Sep; 32(3):906-12.

V. Haugvik SP, Vodák D, Haugom L, Hovig E, Gladhaug IP, Heim S, Micci F.

Transcriptomic profiling of tumor aggressiveness in sporadic nonfunctioning pancreatic neuroendocrine neoplasms. Pancreas. 2016 Feb. Epub ahead of print. 


\section{Introduction}

\section{Historical notes}

In November 1902, the first report of an endocrine pancreatic tumor was published by the Canadian pathologist Albert George Nicholls (1870-1946) ${ }^{1}$. In September 1907, the German pathologist Siegfried Oberndorfer (1876-1944) presented his observations on the nature of a morphologically distinct class of tumors which he referred to as carcinoids $^{2}, 3$, i.e., carcinoma-like neoplasms behaving like benign neoplasms. He thereby became the first to characterize neuroendocrine neoplasms (NENs). In May 1927, the American physician Russell M. Wilder (1885-1959) described the first case of insulinoma and was the first to report a surgical attempt on removal of a pancreatic neuroendocrine neoplasm (PNEN), which was undertaken by the surgeon William James Mayo (1861-1939) ${ }^{4}$. Two years later, the Canadian physician Goldwin Howland (1875-1950) described the first curative operation for a PNEN 5 . Some years later, in 1938, the Austrian pathologist Friedrich Feyrter (1895-1973) published a paper where he proposed that neuroendocrine neoplasms are derived from cells of the diffuse endocrine system ${ }^{6}$. In 1963, the British pathologist Merton Sandler (1926-2014) was the first to classify neuroendocrine neoplasms according to the embryonic divisions of the digestive tract, i.e., foregut, midgut and hindgut ${ }^{7}$. In 1966, the British pathologist Anthony G.E. Pearse (1916-2003) recognized the uptake of 5-hydroxytryptophan (5-HTP) and its decarboxylation to 5-HT as a common cytochemical characteristic in a distinct population of endocrine cells ${ }^{8}$. These cells did not only include cells of the diffuse endocrine system, but also cells of several endocrine organs. $\mathrm{He}$ defined them as amine precursor uptake and decarboxylation (APUD) cells and thereby became the first to classify neuroendocrine cells ${ }^{9}$. All these contributions, made over a time span of seven decades, represent the early era of neuroendocrine oncology and surgical treatment of PNENs, and form the basis of our understanding of how neuroendocrine neoplasms develop and behave. 


\section{Pancreatic neuroendocrine neoplasms (PNENs)}

\section{Definition, clinical presentation, and epidemiology}

PNENs arise from the endocrine cells of the pancreas, which are part of the diffuse endocrine system ${ }^{10}$. They represent a heterogeneous group of diseases and comprise about five percent of all pancreatic neoplasms ${ }^{11,12}$ (Figure 1). Multiple terms for the same group of diseases were suggested, e.g., pancreatic carcinoid, islet cell tumor, pancreatic endocrine tumor, pancreatic neuroendocrine tumor, pancreatic neuroendocrine neoplasm ${ }^{13}$. For reasons of clarity, the general term "pancreatic neuroendocrine neoplasm" or "PNEN" is used in this thesis.
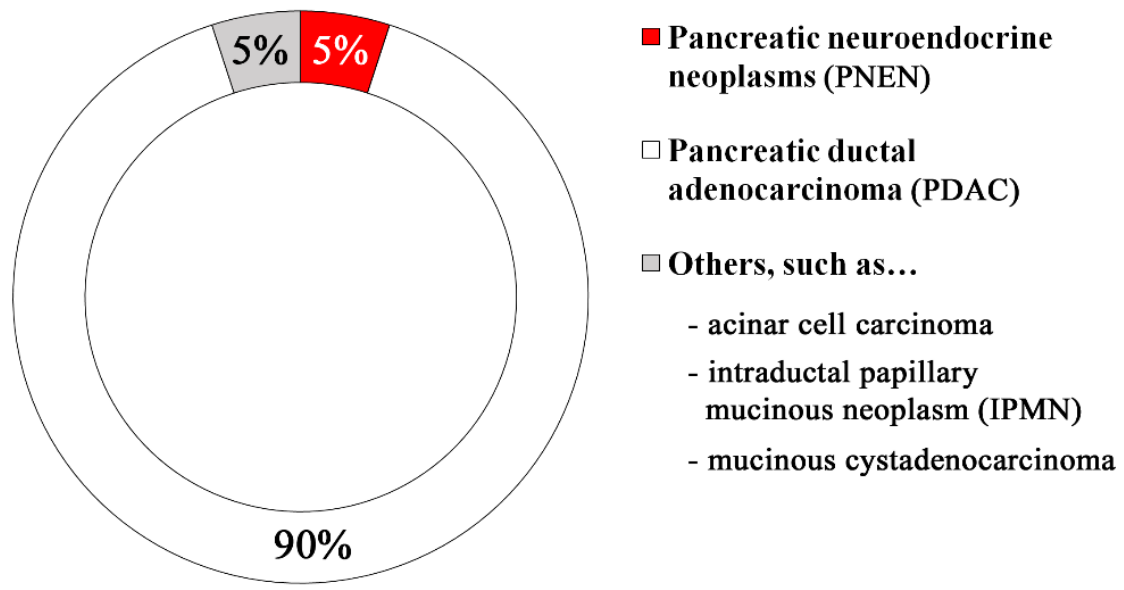

Figure 1. Relative frequency of pancreatic neoplasms in humans

PNENs are clinically diverse and are divided into functioning and nonfunctioning disease, depending on their ability to give symptoms due to hormone production ${ }^{14}$. Sixty to $90 \%$ of all PNENs are nonfunctioning $11,15,16$ as they do not cause hormone-dependent symptoms (Figure 2). Since nonfunctioning PNENs do not cause characteristic hormonal symptoms and generally exhibit slow growth, they are often detected incidentally or through symptoms related to mass effects resulting from local or distant tumor progression ${ }^{17}$. Common symptoms and signs of nonfunctioning PNENs are abdominal pain, nausea, fatigue, obstructive jaundice, and abdominal mass ${ }^{18,19}$. Patients with functioning PNENs often present with characteristic symptoms dependent on the hormones produced, such as 
hypoglycemia (insulin in insulinoma), heartburn (gastrin in gastrinoma), and watery diarrhea (vasoactive intestinal peptide in VIPoma). Functioning PNENs will not be discussed further in this thesis.

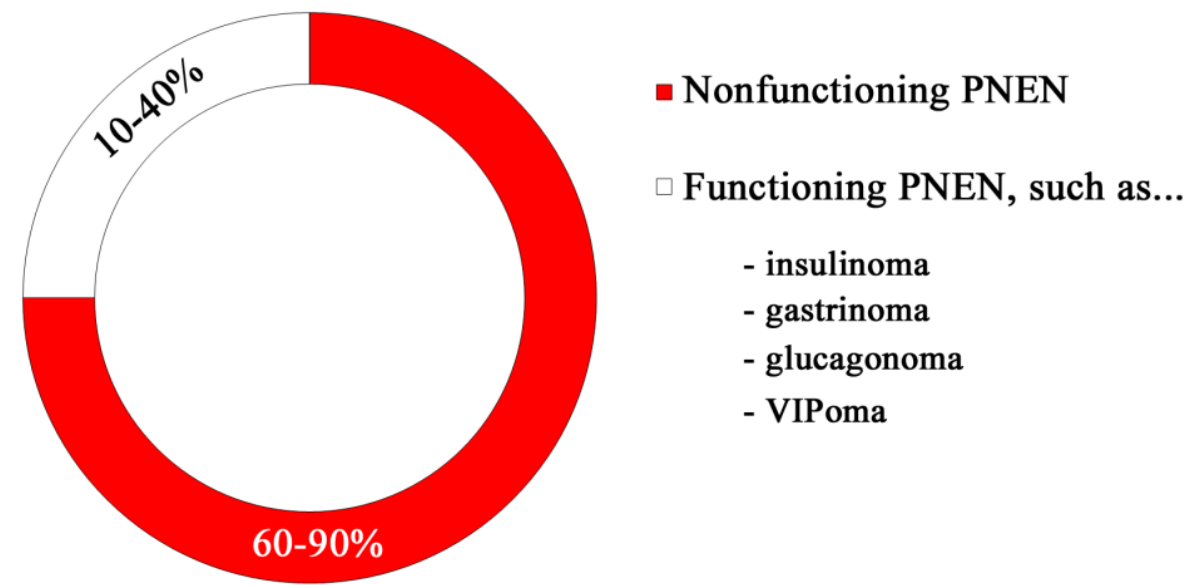

Figure 2. Relative frequency of nonfunctioning and functioning pancreatic neuroendocrine neoplasms (PNENS)

Most PNENs are sporadic, which means that they do not show any specific gene mutation resulting in their occurrence in specific families according to defined inheritance patterns. However, about 10-15\% of all PNENs develop as part of familial syndromes associated with specific germline mutations, such as multiple endocrine neoplasia type 1 syndrome (MEN-1 syndrome, caused by mutation in the MEN1 gene in chromosome subband 11q13.1), von Hippel-Lindau syndrome (VHL syndrome, caused by mutation in the VHL gene in chromosome subband 3p25.3), neurofibromatosis type 1 (NF-1 or von Recklinghausen disease, caused by mutation in the $N F 1$ gene in chromosome subband 17q11.2), and tuberous sclerosis complex (TSC, caused by mutation in the TSC1 or TSC2 gene in chromosome subbands $9 \mathrm{q} 34.13$ and $16 \mathrm{p} 13.3$, respectively $)^{20}$. The relative frequency of sporadic and familial PNENs is illustrated in Figure 3.

Most PNENs are solitary and located in the pancreatic head (35\%), tail (30\%), or body (10\%). About $15 \%$ of all PNENs are multiple. The relative distribution of PNENs in the pancreatic gland is illustrated in Figure 4. 


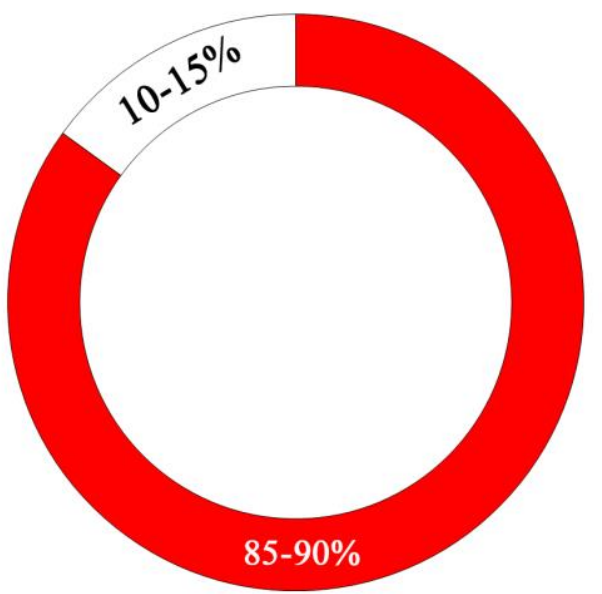

- Sporadic PNEN

$\square$ Familial PNEN, such as...

- multiple endocrine neoplasia type 1 (MEN-1)

- von Hippel-Lindau syndrome (VHL)

- neurofibromatosis type 1 (NF-1)

- tuberous sclerosis complex (TSC)

Figure 3. The relative frequency of sporadic and familial pancreatic neuroendocrine neoplasms (PNENs)

In Norway and in the USA, the median age at diagnosis of PNENs is about 60 years with a slight male predilection (55\%) and with an observed increasing incidence rate throughout the last three decades ${ }^{11,16,21-23}$. While the current incidence rate for PNENs in Norway is 0.7 per 100,000 person-years, with an annual increase of about $7 \%^{21}$, the current incidence rate in the USA is 0.3 per 100,000 person-years ${ }^{11}{ }^{24}$. The higher reported incidence in Norway is probably closer to the actual incidence as these data include PNENs classified with "uncertain behavior", which in similar studies have been excluded. Hence, the number of new cases of PNEN to be expected per year in Norway would now be 36.

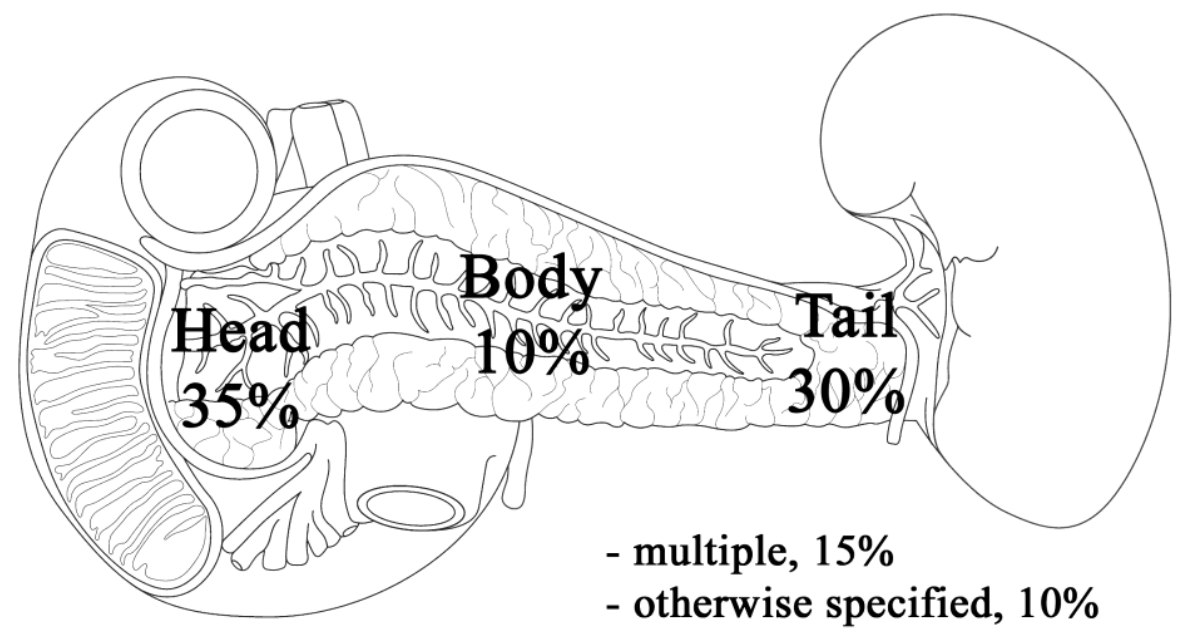

Figure 4. Distribution of PNENs in the pancreas (numbers from Bilimoria et al. ${ }^{25}$ and Fischer et al. ${ }^{26}$; illustration by Haugvik K) 
According to data from the Cancer Registry of Norway, most patients are diagnosed with distant metastatic disease $(52 \%)$, followed by localized disease (29\%) and regional disease (defined by tumor growth into a neighboring structure, including regional lymph nodes) $(19 \%)^{21}$, as illustrated in Figure 5. According to data from the Surveillance, Epidemiology, and End Results (SEER) program, most patients in the USA are diagnosed with distant metastatic disease (64\%), followed by regional disease $(22 \%)$ and localized disease $(14 \%)^{24}$. It is important to notice that the SEER database excludes PNENs considered to be benign, causing overestimation of the frequencies of extrapancreatic disease, nodal metastasis, and metastatic disease ${ }^{27}$. Autopsy studies have shown that PNENs can be identified in as many as $10 \%$ of the population, suggesting that many people carry asymptomatic disease ${ }^{28}$. Whether the generally increasing use of cross-sectional imaging and ultrasound in the last three decades can explain the increase in the incidence of PNENs exclusively, remains unknown ${ }^{22}$.

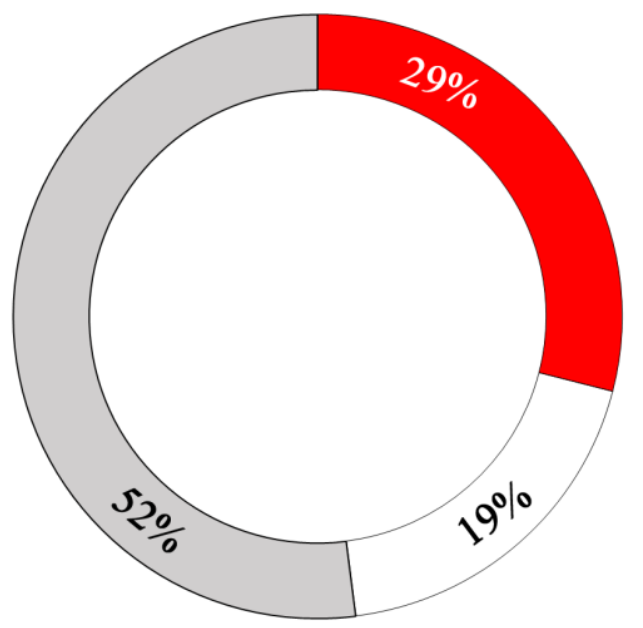

- Local disease

- no disease spread

$\square$ Regional disease

- tumor growth into neighboring structure, including lymph nodes

$\square$ Distant disease

- distant metastases

Figure 5. The relative frequency of tumor stage of patients with PNEN in Norway from 1993 to 2010 (from Boyar Cetinkaya et al. ${ }^{21}$ )

As this introductory chapter shows, most PNENs are nonfunctioning, sporadic tumors located in the pancreatic head, and with synchronous metastatic disease. In addition to classification according to hormonal activity and heredity, PNENs should be further classified in order to enable patient risk stratification and to improve clinical decision making ${ }^{29}$. Today, the prognosis of PNENs is largely 
defined by the individual tumor's morphology, grading, and stage as determined by histopathology.

\section{Pathology}

The pathology of all PNENs is defined by tissue morphology, grading, and the tumor-node-metastasis (TNM) pattern.

Neuroendocrine cells are characterized by production of neurosecretory granules, containing proteins such as chromogranin A and synaptophysin, which can be detected by immunohistochemistry. The minimal immunohistochemical tests recommended for a diagnosis of PNENs, as for GEP-NENs in general, are: chromogranin A, synaptophysin, and Ki67. Chromogranin A and synaptophysin are the two most sensitive and specific general neuroendocrine markers and are used to confirm the diagnosis, whereas $\mathrm{Ki} 67$ is a marker of prognosis that also defines grading ${ }^{13}$. While the characterization of neuroendocrine cell morphology and evaluation of immunohistochemistry in PNENs remain the domain of pathologists $^{30}$ and as such will not be further discussed in this thesis, surgeons should have a thorough understanding of the grading and TNM staging of PNENs.

PNENs are classified according to their grading, defined by the World Health Organization (WHO) 2010 Classification $^{31}$. The grading is based on the Ki67 index, defined as the ratio between the number of cells in a population positive for Ki67 to the total number of cells studied, or the mitotic index, defined as the ratio between the number of cells in a population undergoing mitosis to the number of all cells observed. Ki67 is a nuclear antigen and cell proliferation marker. The Ki67 index has become one of the most important indicators of tumor aggressiveness in GEP-NENs ${ }^{32}$. In PNENs, as for GEP-NENs in general, a mitotic rate of $<2$ and/or Ki67 index of $\leq 2$ corresponds to a neuroendocrine tumor (NET) G1. A mitotic rate of 2-20 and/or Ki67 index of 2.5-20 characterizes a NET G2, while a mitotic rate and/or Ki67 index of > 20 defines a neuroendocrine carcinoma (NEC) G3 (Table 1). 
Table 1. WHO 2010 grading system for pancreatic neuroendocrine neoplasms (modified from Bosman et al. ${ }^{31}$ ). ${ }^{a} 10 \mathrm{HPP}$, high-power field $=2 \mathrm{~mm}^{2}$, at least 40 fields (at $\times 40$ magnification) evaluated in areas of highest mitotic density. ${ }^{b}$ MIBI antibody, \% of 2000 tumor cells in areas of highest nuclear labeling

\begin{tabular}{lll}
\hline Grade & Mitotic count $(10 \mathrm{HPF})^{\mathrm{a}}$ & Ki-67 index $(\%)^{\mathrm{b}}$ \\
\hline G1 & $<2$ & $\leq 2$ \\
G2 & $2-20$ & $2.5-20$ \\
G3 & $>20$ & $>20$ \\
\hline
\end{tabular}

High-grade PNECs (PNECs, G3) are defined as PNENs with poorly differentiated morphology and a higher proliferation rate than well-differentiated PNETs (G1 and G2). It is important to note that grading of a PNEN is determined by the highest mitotic rate or $\mathrm{Ki} 67$ index, irrespective of whether this is found in the primary tumor or a metastatic deposit. At diagnosis, most PNENs are graded as G1 (55\%), followed by G2 (40\%), and G3 (5\%) $)^{33}$, as shown in Figure 6.

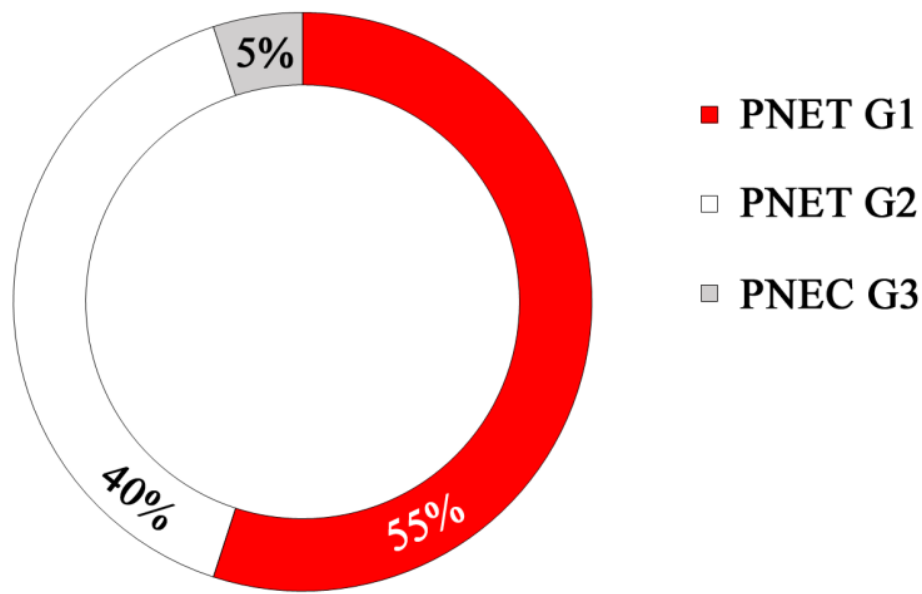

Figure 6. The relative frequency of tumor grading, defined by the WHO 2010 Classification $^{31}$ of patients with pancreatic neuroendocrine neoplasms (PNENs). PNET, pancreatic neuroendocrine tumor; PNEC, pancreatic neuroendocrine carcinoma.

Besides grading, PNENs are classified according to their TNM pattern, as defined by validated TNM staging systems. There are currently two TNM systems for staging of PNENs. The first classification was recommended by the European 
Neuroendocrine Tumor Society (ENETS) in $2006^{34}$ and is predominant in Europe. This was followed by the classification suggested by the American Joint Cancer Committee and International Union for Cancer Control (AJCC/UICC) in $2009^{35}$, which is now widely used in the North American region. The ENETS and AJCC/UICC classification systems for PNENs differ in their definition of the T stage, as shown in Table 2. There is an ongoing debate as to which of the two staging systems is the more precise in terms of prognostic stratifications, with some studies demonstrating similar strength ${ }^{36,37}$ and others indicating superiority of the ENETS over the AJCC/UICC TNM system ${ }^{38,39}$. According to the ENETS staging system, Stage I is defined by T1N0M0, stage IIA by T2N0M0, stage IIB by T3N0M0, stage IIIA by T4N0M0, stage IIIB by anyTN1M0, and stage IV by anyTanyNM1 $1^{34}$. In several European cancer centers, PNENs are most often defined as stage I (28\%) or IV (28\%), followed by IIIB (19\%), IIA (14\%), IIB $(7 \%)$, and IIIA (4\%) at time of diagnosis ${ }^{38}$. In the work contained in this thesis, the ENETS TNM system was used. The prognosis of PNENs, following their ENETS stage and WHO grading, is illustrated in Figure 7 and shows that grading and staging correlate directly with prognosis.

Table 2. Comparison of the T category in the ENETS and AJCC/UICC TNM classifications of pancreatic neuroendocrine neoplasms. ENETS, European Neuroendocrine Tumor Society, AJCC/UICC, American Joint Cancer Committee And International Union for Cancer Control (from Rindi et al. ${ }^{34}$ and Sobin et al. ${ }^{35}$ )

\begin{tabular}{|c|c|c|}
\hline & ENETS TNM & AJCC/UICC \\
\hline $\mathrm{T} 1$ & Confined to pancreas, $<2 \mathrm{~cm}$ & Confined to pancreas, $<2 \mathrm{~cm}$ \\
\hline $\mathrm{T} 2$ & Confined to pancreas, $2-4 \mathrm{~cm}$ & Confined to pancreas, $>2 \mathrm{~cm}$ \\
\hline T3 & $\begin{array}{l}\text { Confined to pancreas, }>4 \mathrm{~cm} \text {, or invasion of } \\
\text { duodenum or bile duct }\end{array}$ & $\begin{array}{l}\text { Extension beyond pancreas, but without involvement of } \\
\text { celiac axis or superior mesenteric artery }\end{array}$ \\
\hline T4 & Invasion of adjacent organs or major vessels & Involvement of celiac axis or superior mesenteric artery \\
\hline
\end{tabular}




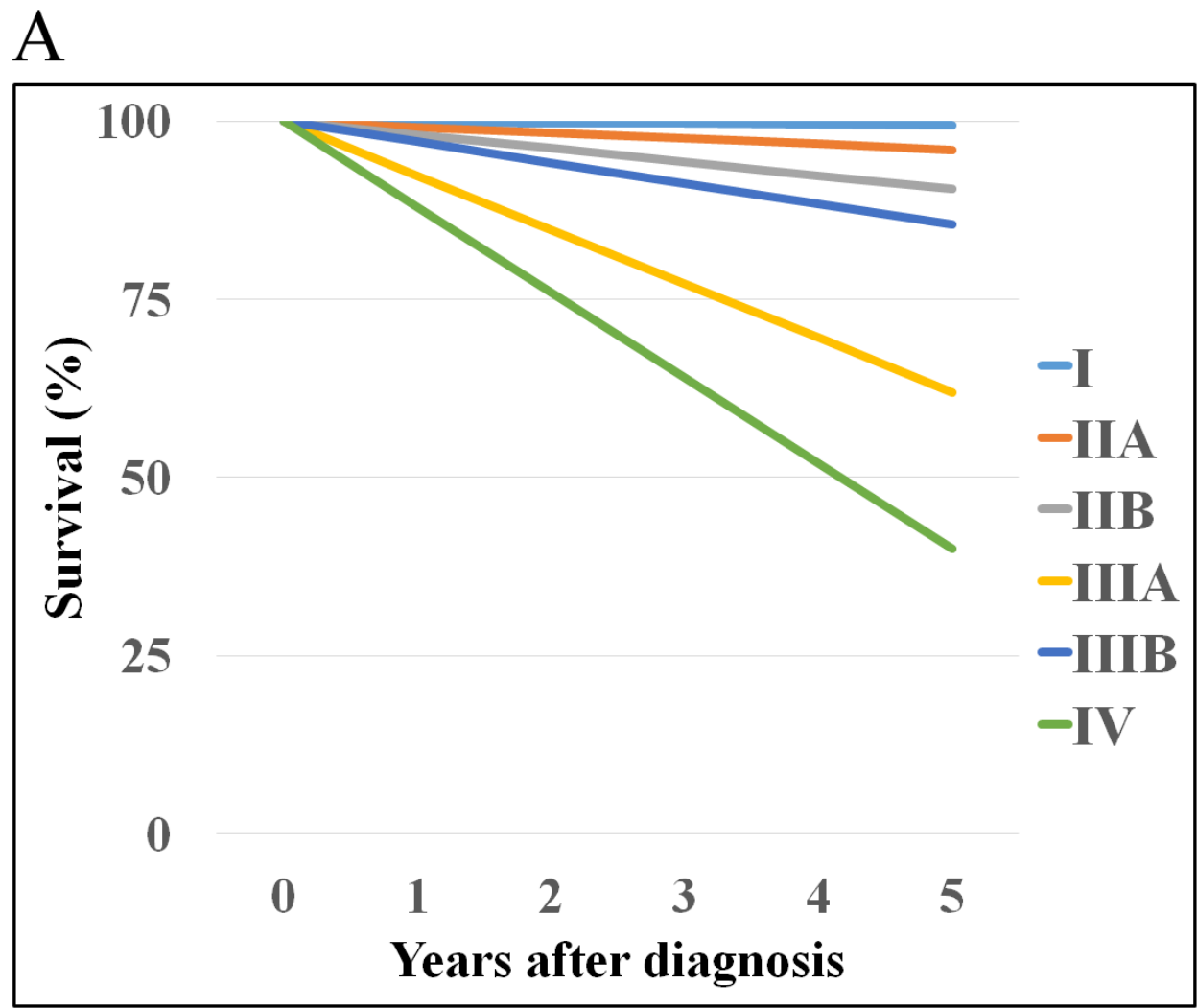

B

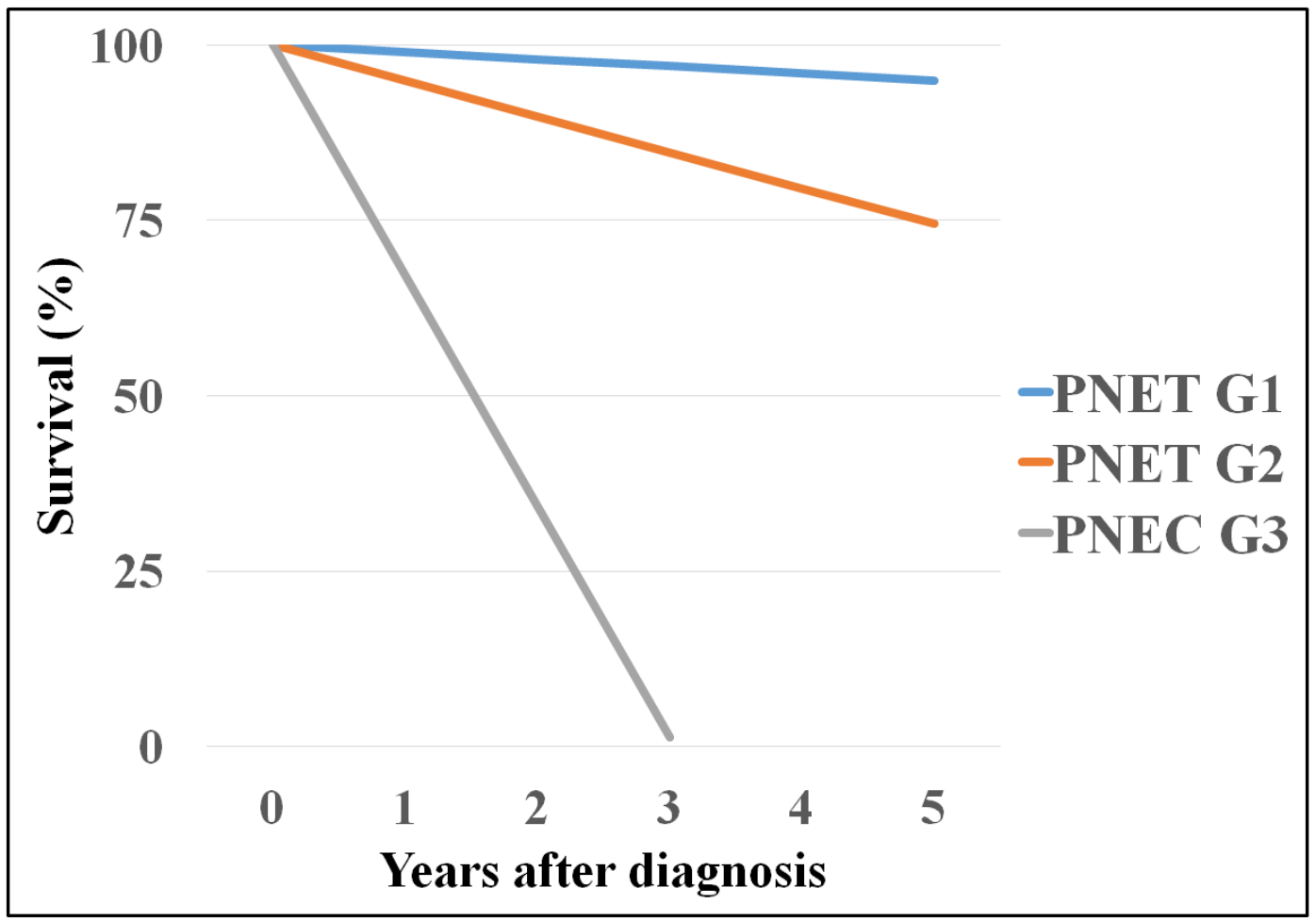

Figure 7. Prognosis of PNENs according to the current (A) ENETS staging and (B) WHO grading (numbers from Rindi et al. ${ }^{38}$ ) 
As shown in this chapter, current classification systems for PNENs are defined by histopathology. The Ki67 index cut-off-values between the different grading classes have proven to correlate well with prognosis of NENs in different organs, including the pancreas ${ }^{40-42}$. However, a substantial fraction of PNENs do not show the prognosis predicted by their corresponding grading and stage. This implies the possibility of future revisions of current classification systems as new knowledge about the different subtypes of PNENs is acquired. In particular, this is to be expected in the group of PNET G2 and the rare group of PNEC, both of which have a wide Ki67 index range. This is exemplified by the recent discourse related to the optimal Ki67 index cut-off between PNET G1 and G2 ${ }^{43}$, 44, which is discussed in Paper I of this thesis. Another example is the discussion related to discordance of tissue morphology and grading in PNENs with well-differentiated morphology and a Ki67 index above $20 \%{ }^{45}$. Beyond histopathology, there is also a need for development of platforms for molecular staging in patients with PNENs.

In Papers IV and $\mathbf{V}$ of this thesis, initial steps toward a molecular staging in patients with sporadic nonfunctioning PNENs were taken using genomic profiling techniques.

\section{Surgery for PNENs}

Modern pancreatic surgery is characterized by both minimally-invasive and highly invasive procedures, which allow the surgeon to remove benign or malignant pancreatic disease at different stages. In the case of PNENs, the expression "modern surgical treatment" may be more relevant than for any other pancreatic neoplasm. Due to slow growth and frequently found small indolent lesions, parenchyma-sparing techniques are warranted ${ }^{46}$. On the other hand, slow growth of metastatic tissue allows surgery of the primary tumor only or of both the primary tumor and metastatic tissue, with evidence of prolonged survival compared to nonsurgical treatment ${ }^{47-50}$. This is especially important for patients with metastatic functioning disease ${ }^{51,52}$. PNENs are generally associated with a favorable prognosis after surgery ${ }^{24}$ as demonstrated by reports of an overall 10 -year survival of up to $40 \%{ }^{25}$. This is in sharp contrast to the more common and highly aggressive 
pancreatic ductal adenocarcinoma (PDAC) with an expected median overall survival of around two years after surgery ${ }^{53}, 54$. Survival among patients with PNENs has improved over the last decades ${ }^{11}$ and improvements in the field of surgery are likely to have contributed substantially to this. This becomes clear as surgical removal is the only curative treatment for patients with PNENs and improves survival compared to nonoperative treatment ${ }^{55}$. Hence, surgery has become a cornerstone treatment modality for patients with PNENs ${ }^{18,56-60}$ with increasing use over the last decades ${ }^{16}$, as illustrated in Figure 8.

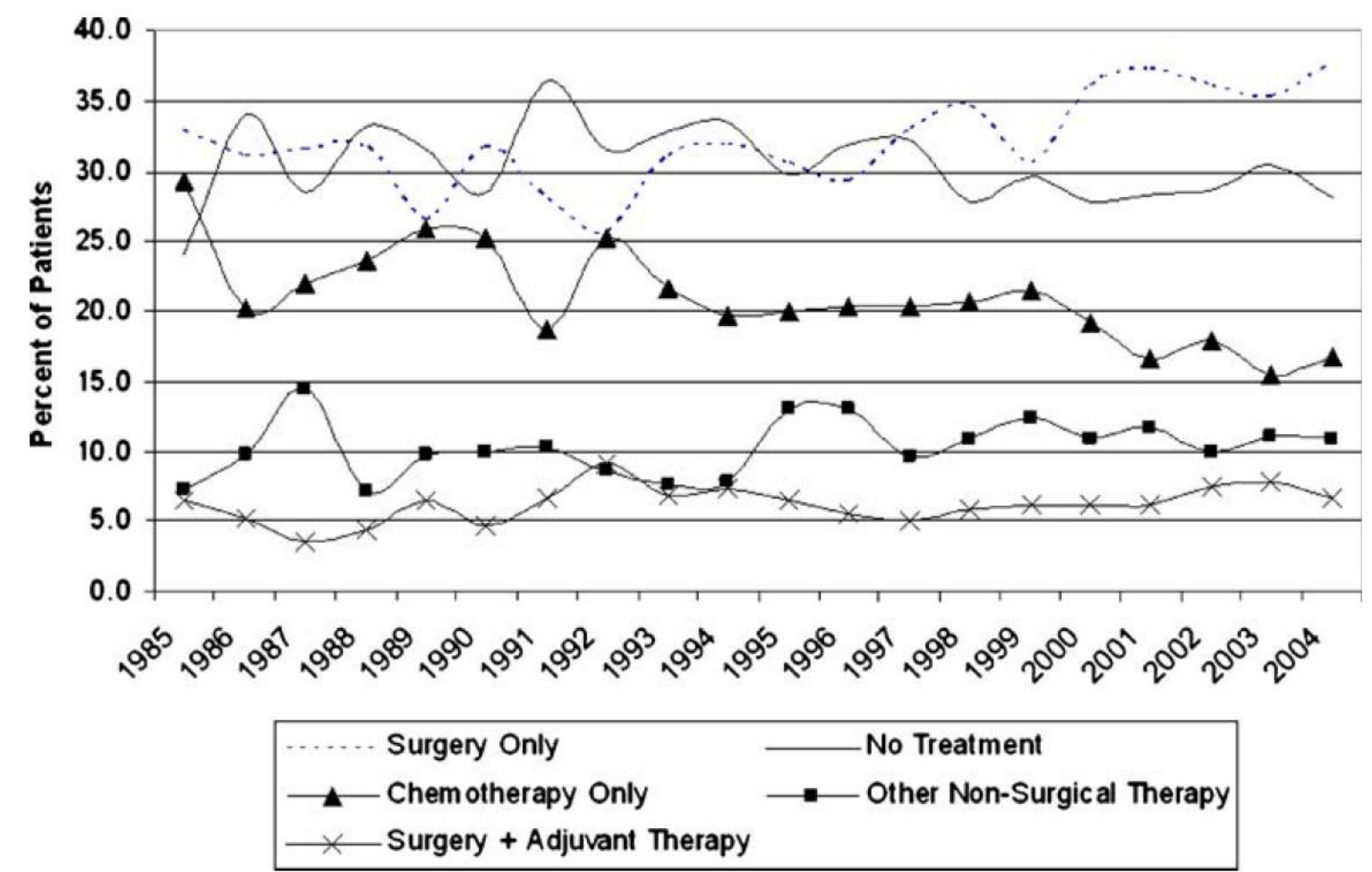

Figure 8. Treatment trends for patients with PNENs from 1985-2004 in the USA (from Bilimoria et al. ${ }^{16}$ )

The goals of surgical treatment for PNENs are cure, relief from hormonal symptoms caused by functioning tumors ${ }^{51}$, or relief from nonfunctioning tumors causing symptoms related to mass effect (e.g., biliary obstruction, gastric outlet obstruction, abdominal pain, or gastrointestinal hemorrhage). Resectability rates up to $60 \%$ have been reported among patients diagnosed with $\mathrm{PNEN}^{55}$, and the resectability rate at our institution is about $50 \%{ }^{60}$. The most common standard surgical procedures for PNENs include pancreatico-duodenectomy (Whipple 
procedure), distal pancreatectomy, and enucleation ${ }^{26,61,62}$. Middle segment pancreatectomy is an alternative for lesions located in the pancreatic neck or body $^{63,64}$, and total pancreatectomy is an alternative for lesions affecting all parts of the organ ${ }^{65,66}$. Enucleation and middle segment pancreatectomy are examples of parenchyma-sparing procedures.

A general risk of standard pancreatic resections (pancreatico-duodenectomy and distal pancreatectomy) is functional impairment of the organ due to loss of parenchyma, resulting in exocrine and/or endocrine insufficiency. Pancreatic exocrine insufficiency is characterized by symptoms related to maldigestion such as steatorrhoea and weight loss due to deficiency of exocrine pancreatic enzymes, whereas pancreatic endocrine insufficiency is associated with development of diabetes mellitus secondary to loss of insulin-producing pancreatic tissue. Parenchyma-sparing procedures, such as enucleation and middle segment pancreatectomy, aim at reducing such side effects ${ }^{46}$.

The first laparoscopic operation for a PNEN was performed in 1992 by the Canadian surgeon Michel Gagner ${ }^{67,68}$. Since then, there has been a general trend towards minimally-invasive techniques in the management of PNENs, especially with laparoscopic procedures. As the laparoscopic approach in pancreatic surgery was proven feasible ${ }^{69-72}$, the advantages of this minimally-invasive surgery slowly led to an increasing number of standard laparoscopic resections and parenchymasparing procedures of benign pancreatic lesions or lesions with low malignant potential, including PNENs ${ }^{73-79}$. Today, we know that the general advantages of the laparoscopic compared to the open approach in pancreatic surgery are less intraoperative bleeding ${ }^{80}$, faster postoperative recovery ${ }^{81}$, shorter hospital stay $^{74}$, ${ }^{76}$, and improved cosmesis.

Most studies describing laparoscopic pancreatic surgery have been focusing more on technical aspects and feasibility of the procedures rather than the underlying pancreatic disease. Hence, while we now have learned that laparoscopic pancreatic 
surgery is feasible, knowledge of laparoscopic pancreatic surgery in patients with PNENs is limited. At the beginning of this thesis, only few large series of patients undergoing laparoscopic surgery for PNENs had been published ${ }^{77,82,83}$. In order to increase the knowledge about minimally-invasive surgery for this rare group of patients, we reported what at the time of publication was the largest single center series of patients undergoing laparoscopic surgery for PNENs (Paper I of this thesis).

Besides evolutions in surgical care, anesthesiology and intensive care medicine have developed rapidly over the last few years and now allow highly invasive approaches in pancreatic surgery without compromising perioperative patient survival. As some PNENs are large and infiltrate adjacent organs, i.e, show local advancement needing multivisceral resection and/or vascular reconstruction, highly invasive pancreatic surgery may be required.

There is no uniformly accepted definition of "locally advanced" disease for PNENs. Therefore, in this thesis, we defined locally advanced disease as a PNEN with an ENETS T3- (confined to pancreas, $>4 \mathrm{~cm}$, or invasion of duodenum or bile duct) or T4-stage (invasion of adjacent organs or major vessels) ${ }^{34}$. Surgical treatment of locally advanced PNENs is controversial ${ }^{84}$ and some regard vascular infiltration as a contraindication for resection ${ }^{85}$. There are only a few reports that include vascular reconstruction among patients with PNENs, and none of these discuss the role of vascular reconstruction as such ${ }^{84,86-96}$. This is different from the more common and generally much more aggressive locally advanced PDAC, where the concept of vascular reconstruction has already been discussed widely ${ }^{97-}$ 100 and has been associated with acceptable morbidity, mortality, and better overall survival as compared to unresected patients ${ }^{101}$. Hence, discussion on the role of vascular reconstruction in locally advanced PNEN seems to be warranted. In Paper II of this thesis, the role of vascular reconstruction in a small series of patients with locally advanced PNENs was assessed. 
Based on what has been presented so far in the introductory chapters of this thesis, it is clear that PNENs constitute a rare, diverse, and medically challenging group of diseases that require multidisciplinary attention at specialized institutions in order to optimize patient treatment and outcome ${ }^{85,102-105}$. While around half of all PNENs are resectable, as described earlier, the other half of patients will most probably need other or additional treatment modalities such as systemic chemotherapy, molecular therapy (e.g., everolimus and sunitinib), biotherapy with long-acting somatostatin analogs, radiotherapy, peptide receptor radionuclide therapy, and/or locoregional interventional treatment of metastatic disease ${ }^{15,} 106$. For the latter group of patients, there are currently no evidence-based treatment sequences that involve surgery and attempts to develop such approaches are thus urgently needed. As surgery can be considered a treatment modality at all stages of PNENs, the surgeon plays an essential role in the multidisciplinary team. Although the clinical research included in this thesis belongs to the field of surgery, the results from each of the studies should be considered as matters for multidisciplinary discussions.

\section{High-grade pancreatic neuroendocrine carcinoma (PNEC)}

During the last two decades, notable progress has occurred in basic, translational, and clinical research on PNETs ${ }^{107,108}$. At the same time, as described in the sections above, there has been a general trend towards both more minimallyinvasive and highly invasive surgery of these patients ${ }^{78,85,91,109-111}$. In contrast, patients with PNECs have not gained similar attention.

In Norway, the incidence of PNECs has remained stable through the past two decades with an incidence rate of approximately 0.04 per 100,000 person-years ${ }^{21}$. The tumors are most frequently diagnosed in patients around 60 years of age, with a male predilection (59\%) and a predominance of tumors located in the pancreatic head $(61 \%)^{112}$. The tumors are characterized by poorly differentiated morphology and a higher proliferation rate than well-differentiated PNETs. 
In contrast to the indisputable importance of surgery as a treatment option for patients with PNETs, the role of surgery in the treatment of PNEC remains unclear. This may be explained by the common presence of synchronous metastatic disease and the rapid progression of PNECs, as illustrated in Figure 7, which traditionally has been seen as necessitating palliative systemic chemotherapy ${ }^{113}$. However, less than half of the PNEC patients respond to such treatment regimens ${ }^{113}$ and alternative treatment options are urgently needed.

The current consensus guidelines of the ENETS for the surgical management of patients with gastro-entero-pancreatic NECs (GEP-NECs) refer to only three studies $^{114-116}$, out of which only one case report discussed surgery of PNEC as such $^{116}$. The guidelines state that localized disease should be treated with surgery or radiotherapy and platinum-based chemotherapy, whereas surgical resection of metastasis is not recommended ${ }^{106}$. The North American Neuroendocrine Tumor Society (NANETS) guidelines state that the benefit of surgery among patients who have completed a course of chemoradiation is uncertain, but reference no studies on surgery for PNECs ${ }^{117}$, 118 . Surgery is not even mentioned in the section on treatment for metastatic PNEC. Moreover, the European Society for Medical Oncology's (ESMO) guidelines state that there is general agreement not to operate on PNECs ${ }^{119}$. The current international consensus guidelines on surgical treatment for PNECs are based on expert opinions and very little evidence. This underscores the importance of defining the role of surgery in patients with PNEC by conducting clinical research ${ }^{120}$. In Paper III of this thesis, we have described the first comparative study on effect of combined surgical treatment and chemotherapy against chemotherapy alone, in patients with PNEC.

\section{Genetics of PNENs}

GEP-NENs share similar histological and morphological features. However, PNENs are characterized by a distinct genetic basis and corresponding biological behavior. As cancer in general, PNEN is the phenotypic result of the acquisition of one or more genomic changes taking place at the chromosomal and/or gene 
level ${ }^{121}$. As mentioned earlier, some patients are diagnosed with PNEN in the context of familial syndromes caused by specific genetic alterations. These syndromes and their causal genetic patterns serve as reference models for the study of the much more common sporadic PNENs, as the same genes might be mutated in sporadic cases. Interestingly, most PNENs found in patients with familial syndromes are nonfunctioning ${ }^{122}$.

\section{Familial syndromes}

As many as $10 \%$ of all PNENs occur as part of a multiple endocrine neoplasia type 1 syndrome (MEN-1 syndrome), which is the most common familial syndrome related to PNENs. The MEN-1 syndrome is an autosomal dominant disorder clinically associated with predisposition to neoplasms of the parathyroid glands, anterior pituitary, and neuroendocrine pancreatic cells ${ }^{123}$. It is caused by inactivating mutations in the MEN1 gene, which is a tumor suppressor gene in chromosome subband 11q13.1. MEN1 encodes menin, a protein that inactivates transcription factors at the nuclear level, modulates cell cycle inhibitors, and interacts with the DNA repair process. These changes result in inhibition of the cell cycle. PNENs develop in up to $100 \%$ of patients with the MEN-1 syndrome.

The von Hippel-Lindau syndrome (VHL syndrome) is an autosomal dominant disorder characterized by at least one of the following: pheochromocytoma, renal cell carcinoma, retinal or cerebellar hemangioblastoma, and other less frequent neoplasms such as PNENs ${ }^{124}$. PNENS develop in up to $17 \%$ of patients with the VHL syndrome ${ }^{125}$. It is caused by inactivating mutations in the VHL gene, which is a tumor suppressor gene in chromosome subband 3p25.3. The VHL gene encodes for the protein VHL that inactivates angiogenesis via the $\mathrm{PI} 3 \mathrm{~K} / \mathrm{Akt} / \mathrm{mammalian}$ target of rapamycin (mTOR) pathway.

Neurofibromatosis type $1(N F-1)$ is one of the most common inherited disorders and shows an autosomal dominant inheritance pattern ${ }^{126}$. The syndrome is defined by multiple café-au-lait skin spots and neurofibromas, and carries a relatively high 
risk of development of various malignant diseases, including PNENs. However, PNENs develop in very few patients with the NF-1 syndrome. It is caused by inactivating mutations of the $N F 1$ gene in 17q11.2, which codes for neurofibromin. Neurofibromin is a negative regulator of the Ras pathway, and in particular of mTOR function, which prevents overactivation of the mTOR pathway. Hence, cell proliferation is controlled.

The tuberous sclerosis complex (TSC) is an autosomal dominant disorder characterized by typical skin lesions, renal angiomyolipomas, hamartomas, mental retardation, and neurological disorders ${ }^{127}$. PNENs are very rarely associated with TSC. It is caused by mutations of the TSC1 gene in chromosome subband 9q34.13 and the TSC2 gene in 16p13.3 which encode hamartin and tuberin, respectively. Both proteins control cell proliferation through interaction with the $\mathrm{PI} 3 \mathrm{~K} / \mathrm{Akt} / \mathrm{mTOR}$ pathway and insulin receptor signaling.

\section{Sporadic PNENs and altered signaling pathways}

While 10-15\% of all PNENs diagnosed are linked to a familial syndrome, most PNENs occur sporadically. Studies on sporadic PNENs have shown a relatively high frequency of genomic imbalances on chromosome arms 11q, 6q, 11p, 3p, 1p, 10q, 1q, 17q, 7q, 20q, 9p, 7p, and 9q ${ }^{128}$. Some of these chromosomal locations correspond to the gene loci of MEN1 (11q), VHL (3p), and NF1 (17q), suggesting a possible relationship to mutations seen in familial syndromes. This has been further investigated by means of high throughput DNA sequencing, which has shown that around $40 \%$ of sporadic PNENs show mutations in the MEN1 gene, around $10 \%$ show mutations in the TSC gene, whereas mutations in the VHL gene rarely occur ${ }^{129}$. However, as genomic imbalances have also been detected at several other chromosomal locations, further genetic alterations in sporadic PNENs should be expected. DNA sequencing has shown that the most commonly mutated genes in sporadic PNENs encode proteins that are involved in chromatin remodeling, such as $M E N 1, D A X X(6 \mathrm{p} 21.32)$, and $A T R X(\mathrm{Xq} 21.1)^{129}$. As many as $45 \%$ of sporadic PNENs show mutations in either DAXX or ATRX. 
Genetic research on PNENs has thus shown that there is a correlation between mutated genes and corresponding gene products in certain signaling pathways. These pathways include the chromatin remodeling pathway, PI3K/Akt/mTOR pathway, and the TP53/Rb pathway ${ }^{122}$ as listed in Table 3. The chromatin remodeling pathway involves $D A X X, A T R X$ and $M E N 1$. As DAXX and ATRX encode proteins that are responsible for chromatin remodeling, mutations in these genes may lead to chromosomal instability resulting in further mutations and chromosomal abnormalities eventually promoting tumor progression ${ }^{130}$. MEN1 mutations cause cell proliferation through altered signaling of different chromatin modification complexes.

Table 3. Altered signaling pathways in pancreatic neuroendocrine neoplasms (PNENs) (from Shi et al. ${ }^{122}$ )

\begin{tabular}{|c|c|c|c|c|}
\hline $\begin{array}{l}\text { Signaling } \\
\text { pathway }\end{array}$ & $\begin{array}{l}\text { Genes or } \\
\text { molecules }\end{array}$ & Frequency & Possible mechanisms & $\begin{array}{l}\text { Clinical } \\
\text { significance }\end{array}$ \\
\hline \multirow{2}{*}{$\begin{array}{l}\text { Chromatin } \\
\text { remodeling }\end{array}$} & DAXX/ARTX & $45 \%$ & Loss of DAXX/ARTX $\rightarrow \mathrm{ALT} \rightarrow \mathrm{CIN}$ & Prognosis \\
\hline & MEN1 & $50 \%$ & $\begin{array}{l}\text { (1) Loss of menin } \rightarrow \text { decreased histone H3K } 4 \\
\text { methylation } \rightarrow \text { decreased expression of CDKIs } \rightarrow \\
\text { increased cell proliferation } \\
\text { (2) Loss of menin } \rightarrow \text { decreased histone deacetylation } \rightarrow \\
\text { decreased interaction with JunD } \rightarrow \text { cell proliferation }\end{array}$ & Prognosis \\
\hline \multirow[t]{4}{*}{$\begin{array}{l}\mathrm{PI} 3 \mathrm{~K} / \mathrm{Akt} / \\
\mathrm{mTOR}\end{array}$} & $\begin{array}{l}\text { PIK3CA, PTEN, } \\
\text { and TSC2 }\end{array}$ & $15 \%$ & $\begin{array}{l}\text { (1) Mutation in PIK3CA } \rightarrow \text { activation of downstream } \\
\mathrm{Akt} / \mathrm{mTOR}\end{array}$ & $\begin{array}{l}\text { Therapeutic } \\
\text { targets }\end{array}$ \\
\hline & $\begin{array}{l}\text { Growth factors } \\
\text { Growth factor } \\
\text { receptors }\end{array}$ & Majority & $\begin{array}{l}\text { (2) Mutations in PTEN/TSC2 } \rightarrow \text { loss negative regulation } \\
\text { of the PIK3/Akt/mTOR pathway } \\
\text { (3) Overexpression of growth factors/growth factor } \\
\text { receptors } \rightarrow \text { increased activation of the pathway }\end{array}$ & \\
\hline & MEN1 & $50 \%$ & $\begin{array}{l}\text { Loss of menin } \rightarrow \text { loss inhibitory effect on IGFBP } 2 \rightarrow \\
\text { activation of IGF-associated PI3K/Akt/mTOR } \\
\text { activation }\end{array}$ & \\
\hline & VHL & Small subset & $\begin{array}{l}\text { Loss of } \mathrm{VHL} \rightarrow \text { increase HIF- } 1 \alpha \text { (downstream of mTOR) } \\
\text { activity } \rightarrow \text { angiogenesis }\end{array}$ & \\
\hline \multirow[t]{5}{*}{$\mathrm{TP} 53 / \mathrm{Rb}$} & TP53 & $\begin{array}{l}\text { Mutation: common in PD- } \\
\text { NECs }\end{array}$ & $\begin{array}{l}\text { (1) Loss of TP53 } \rightarrow \text { entry into cell cycle (cell } \\
\text { proliferation) and decreased cell apoptosis }\end{array}$ & $\begin{array}{l}\text { Potential } \\
\text { therapeutic }\end{array}$ \\
\hline & & $\begin{array}{l}\text { Overexpression of MDM2/ } \\
\text { MDM4/WIP1: common } \\
\text { in PanNETs }\end{array}$ & (2) Loss of $\mathrm{Rb} \rightarrow$ entry into cell cycle $\rightarrow$ cell proliferation & targets \\
\hline & $\mathrm{Rb}$ & $\begin{array}{l}\text { Mutation: common in PD- } \\
\text { NECs }\end{array}$ & & \\
\hline & & $\begin{array}{l}\text { Overexpression of Cdk4/ } \\
\text { Cdk6: common in }\end{array}$ & & \\
\hline & & PanNETs & & \\
\hline \multicolumn{5}{|c|}{$\begin{array}{l}\text { DAXX, death domain-associated protein gene; ARTX, alpha-thalassemia/mental retardation X-linked gene; ALT, alternative lengthening of } \\
\text { telomere; CIN, chromosome instability; CDKIs, cyclin-dependent kinase inhibitor proteins; MEN1, multiple endocrine neoplasia type } 1 \text { gene } \\
\text { PIK3CA, phosphatidylinositol 4,5-bisphosphate 3-kinase, catalytic subunit alpha gene; PTEN, phosphatase and tensin homolog gene; TSC2 } \\
\text { tuberous sclerosis complex } 2 \text { gene; mTOR, mammalian target of rapamycin; IGFBP2, insulin-like growth factor-binding protein 2; IGF, insulin- } \\
\text { like growth factor; VHL, von Hippel-Lindau tumor suppressor gene; HIF-1 } 1 \alpha \text {, hypoxia-induced factor 1 } \alpha \text {; PD-NECs, poorly differentiated } \\
\text { neuroendocrine carcinoma; PanNET, pancreatic well-differentiated neuroendocrine tumor. }\end{array}$} \\
\hline
\end{tabular}

The PI3K/Akt/mTOR pathway is an intracellular signaling pathway that acts downstream of several receptors and regulates protein translation. It is activated in several types of cancer ${ }^{131}$. About $15 \%$ of sporadic PNENs show mutations in genes of the PI3K/Akt/mTOR pathway ${ }^{129}$, which is also pathogenetically involved in the 
MEN-1 syndrome, VHL syndrome, NF-1, and TSC (Figure 9). Growth factor receptors such as VEGFR and PDGFR normally stimulate the PI3K/Akt/mTOR pathway. In PNENs, these are frequently overexpressed ${ }^{132}$. Activation of this pathway may result in cell proliferation, invasion, or angiogenesis through downstream targets. TSC1/2 and PTEN are two negative regulators of the $\mathrm{PI} 3 \mathrm{~K} / \mathrm{Akt} / \mathrm{mTOR}$ pathway which are often downregulated in PNENs ${ }^{133}$. A third pathway often involved in PNENs is the TP53/Rb pathway, which involves the proteins $\mathrm{p} 53$ and $\mathrm{Rb}$ that are essential parts of tumor-suppressor pathways operative in other cancers. Mutations in TP53 or RB1 are not common in PNETs ${ }^{129}$, but are often seen in PNECs ${ }^{134}$. Interestingly, PNECs do not show mutations in $D A X X$ and $A T R X$. Taken together, these findings suggest that PNECs comprise a genetically distinct subgroup of PNENs.

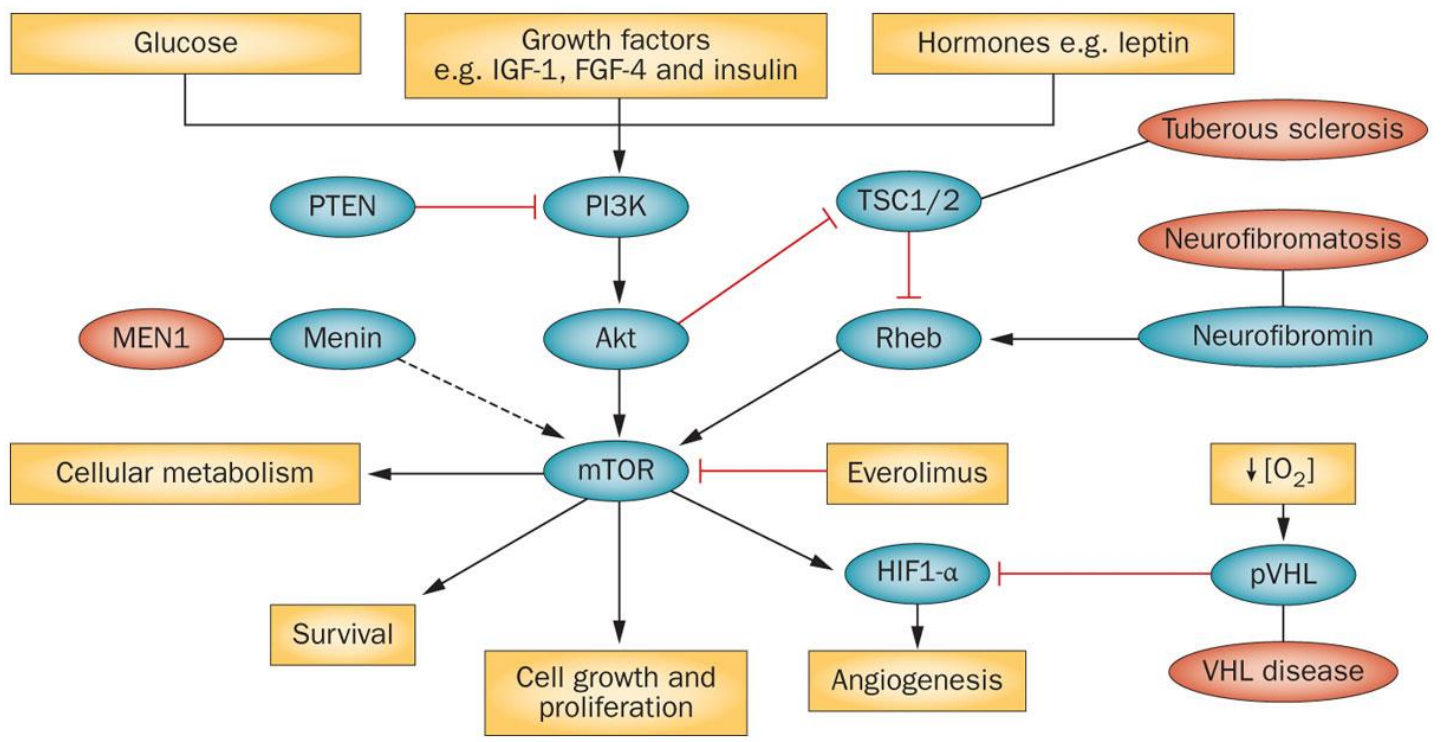

Figure 9. The PI3K/Akt/mTOR pathway (from Oberg et al. ${ }^{135}$ ). Familial syndromes (red) are caused by mutations in these genes. MEN1, multiple endocrine neoplasia type 1 syndrome; VHL disease, von Hippel-Lindau disease (or syndrome).

\section{Cytogenetics and RNA sequencing}

One way of gaining insight into the genetic mechanisms underlying PNENs is by detection of genomic alteration, both structural and numerical, through cytogenetic analyses. Cancer cytogenetics is concerned with the study of genomic alterations 
in malignant disease at the level of chromosomes and/or chromosomal bands ${ }^{121}$. It represents a branch of genetics that involves methods such as karyotyping and comparative genomic hybridization (CGH). Screening of the whole tumor genome by cytogenetic methods is a natural starting point when trying to understand the pathogenetic mechanisms behind tumor development ${ }^{121}$.

Karyotyping is the process of pairing and ordering all the chromosomes of an organism. In somatic cells, the chromosomes are usually studied at the metaphase stage of the cell cycle when chromatin is condensed and the morphology of the chromosomes is clear. In each chromosome, the short (p) and long (q) arms are divided into regions, which are further classified in bands and subbands. By staining techniques, such as G-banding, AT-rich sequences are distinguished from GC-rich sequences.

$\mathrm{CGH}$ is a molecular cytogenetic method that allows identification of genomic imbalances, i.e., segments of the genome that are over- or underrepresented in neoplastic tissue ${ }^{121}$. Patterns of copy number alterations identified by CGH have helped classify tumors into biologically and clinically meaningful subtypes ${ }^{136}$. First, DNA is extracted from the tumor specimen and a normal reference sample. Tumor DNA and normal DNA are then amplified and labeled with fluorophores before they are mixed. This results in complementary target sequences with differences between the tumor and normal reference cells, which can be quantified by digital image analysis, as illustrated in Figure 10. Besides the traditional metaphase $\mathrm{CGH}$, where the target sequences are normal chromosome spreads, array CGH is characterized by target sequences found as DNA fragments fixed in a matrix system. Array CGH enables a higher resolution than does metaphase $\mathrm{CGH}$, but both techniques have limitations inasmuch as they cannot assess intercellular variability or balanced rearrangements such as inversions, insertions, and translocations ${ }^{121}$. The approximate resolution level is more than five megabases for metaphase CGH and more than fifty kilobases for array CGH. In Paper IV of this thesis, we applied metaphase CGH. 


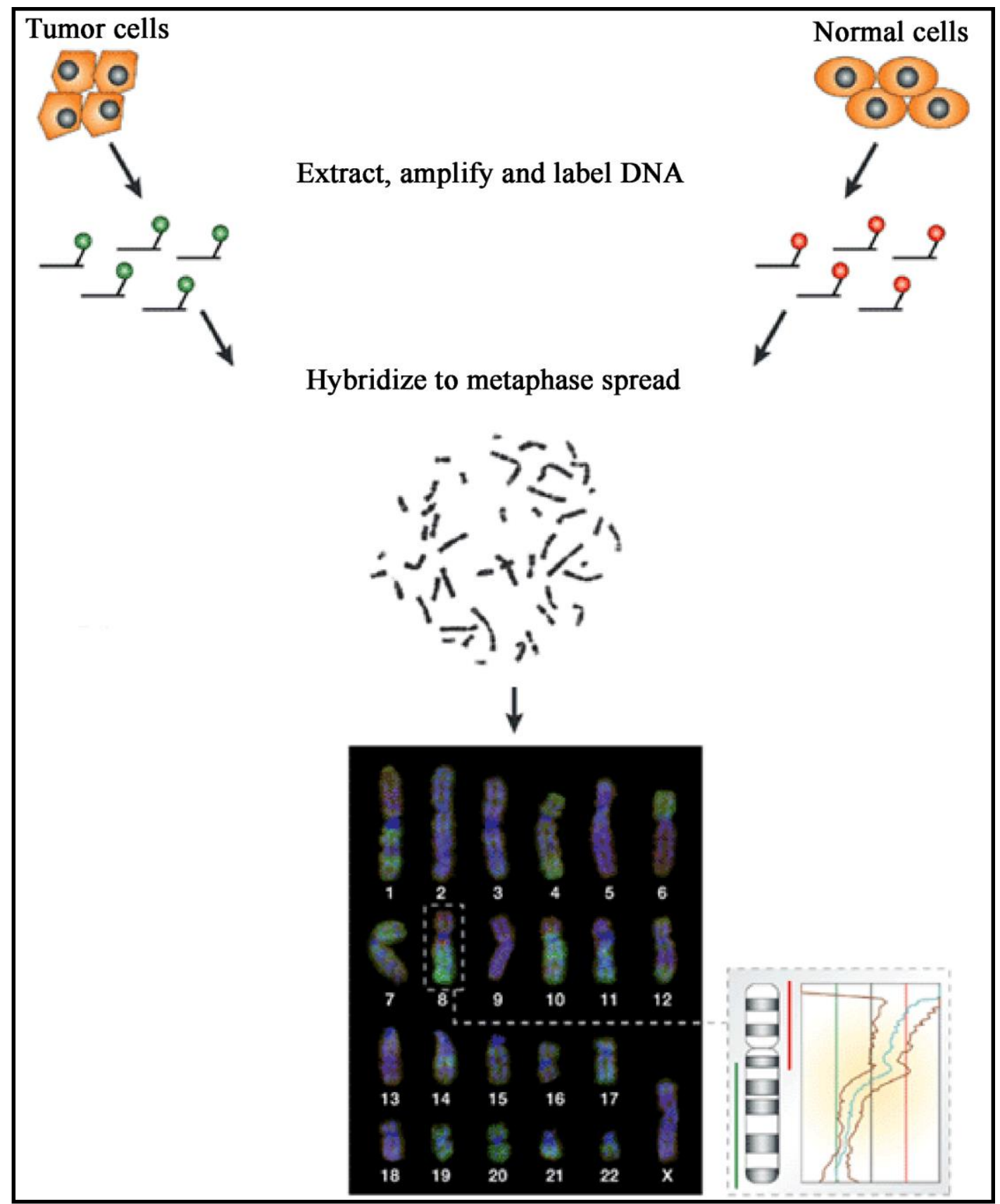

Figure 10. The steps of conventional comparative genomic hybridization (after Chial et al. $\left.{ }^{137}\right)$. Tumor DNA is labeled with green fluorophore and normal DNA is labeled with red fluorophore. Chrosomal regions that were amplified in the tumor tissue appear green and regions that were deleted appear red on the metaphase spread on the bottom left panel

Another way of increasing our knowledge about pathogenetically important genetic changes in PNENs is through genetic expression analysis by methods such as Northern blotting, fluorescent in situ hybridization (FISH), quantitative realtime polymerase chain reaction (PCR), DNA microarray, and high throughput sequencing of RNA. In high throughput RNA sequencing (RNA-seq), RNA is 
converted to complementary DNA or RNA fragments with adaptors attached to one or both ends ${ }^{138}$. Each fragment is amplified by PCR and sequenced in a high throughput manner to gain short sequences from both ends, known as paired-end sequencing. The resulting reads are then aligned to a reference genome or reference transcripts, which allows quantification of the level of expression for each gene (Figure 11).

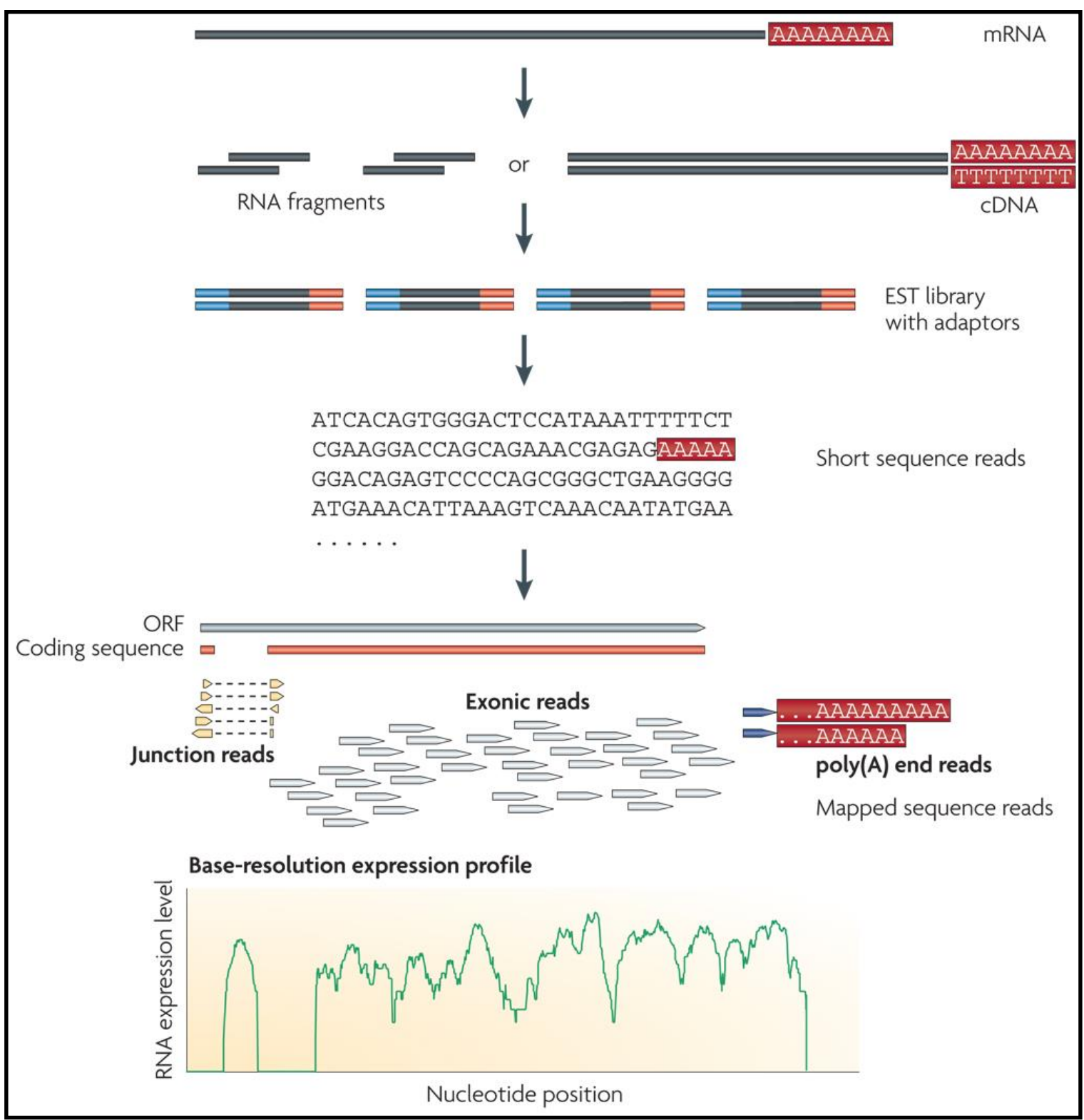

Figure 11. High throughput RNA sequencing (from Wang et al. ${ }^{138}$ ). Sequencing adaptors (blue) are added to each fragment. The resulting sequence reads are classified as three types: exonic reads, junction reads, and poly(A) end-reads. mRNA, messenger RNA; cDNA, complementary DNA; EST, expressed sequence tag; ORF, open reading frame. 
At the beginning of this thesis, the Mitelman Database on Chromome Aberrations and Gene Fusions in Cancer reported seven PNENs with karyotypic aberrations ${ }^{139}$, but no common chromosomal abnormalities ${ }^{140-142}$. Thus, knowledge regarding the chromosomal characteristics of this type of cancer was clearly insufficient. Information on genomic imbalances in nonfunctioning PNENs detected by CGH was limited to 54 cases $^{143-146}$, with common copy number gains of $7 \mathrm{q}, 17 \mathrm{q}$, and 20q, and common copy number losses of 6q, 11p, and 11q. The available CGH data on PNENs had been obtained studying small and heterogeneous series of neoplasms and the findings were therefore difficult to generalize ${ }^{128}$. Furthermore, at the beginning of this thesis, there were only few studies that had investigated gene expression profiles in PNENs ${ }^{133},{ }^{147-153}$, and no consistent patterns of upregulated or downregulated genes had been established ${ }^{154}$. Moreover, there were no published reports on high throughput RNA-seq of sporadic nonfunctioning PNENs.

At present, clinical management of patients with sporadic PNEN is largely based on grading and staging as defined by histopathology. However, as mentioned above, the malignant potential among sporadic PNENs of the same grade and stage may vary considerably. A more precise classification of PNENs, based on molecular characteristics might predict prognosis more precisely. Hence, further knowledge of the molecular pathology of these rare and still poorly understood neoplasms might serve as a starting point for development of such prognostic molecular markers. In Paper IV of this thesis, we performed karyotyping and $\mathrm{CGH}$ in a small series of sporadic nonfunctioning PNENs, in order to identify genomic imbalance patterns that might be important for molecular differentiation of tumor aggressiveness. In Paper V, we performed high throughput RNA-seq in the same series of PNENs in order to identify significant intertumor variations of transcripts of protein-coding genes that may reveal yet unknown molecular markers of prognosis. 


\section{Aims of the thesis}

General aims:

- To investigate different aspects of modern surgical treatment for PNENs

- To identify genomic imbalance and genomic expression patterns that may be important for molecular differentiation of tumor aggressiveness in sporadic nonfunctioning PNENs

Specific aims:

- To describe the feasibility, outcome, and tumor characteristics in a PNEN patient cohort treated with laparoscopic surgery (Paper I)

- To evaluate the prognostic value of the WHO 2010 grading system and ENETS TNM system in a PNEN patient cohort treated with laparoscopic surgery (Paper I)

- To evaluate the feasibility and outcome of pancreatic surgery with vascular reconstruction in patients with locally advanced PNENs

\section{(Paper II)}

- To compare the effect of combined surgical treatment and chemotherapy against chemotherapy alone, in patients with PNEC (Paper III)

- To identify potential prognostic factors for survival in patients with PNEC (Paper III)

- To identify genomic aberration patterns that may be important for molecular differentiation of tumor aggressiveness in sporadic nonfunctioning PNENs (Paper IV)

- To identify significant intertumor variations of transcripts of proteincoding genes in sporadic nonfunctioning PNENs (Paper V) 


\section{Summary of results}

\section{Paper I}

\section{Long-term outcome of laparoscopic surgery for pancreatic neuroendocrine}

tumors.

World J Surg. 2013 Mar; 37(3):582-90.

This paper reports the outcome of 72 patients at a university hospital in Norway between 1997 and 2011 (Figure 12). Sixty-five patients underwent laparoscopic removal of PNEN and their median follow-up was 51 (6-178) months. Overall morbidity was $42 \%$, defined by the revised Accordion Classification, with a surgical morbidity rate of $21 \%$ and postoperative pancreatic fistula (POPF) formation of $21 \%$. A higher rate of POPF was observed in patients undergoing laparoscopic enucleation compared with resection. Five-year disease-specific survival rate was $90 \%$. Statistically significant prognostic factors included T stage, $\mathrm{R}$ stage, and Ki67 expression above the cut-off value of $5 \%$.

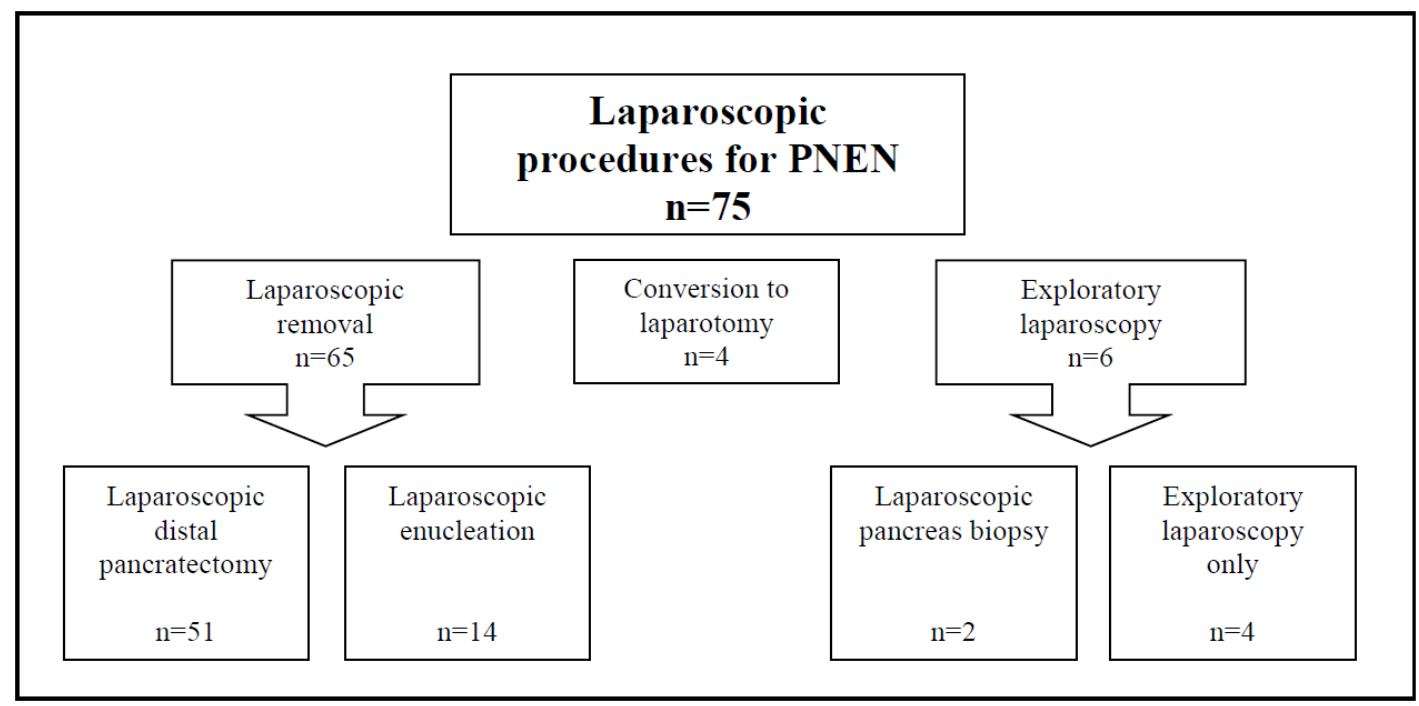

Figure 12. Flowchart of the patients included in Paper I. Three patients had repetitive surgery: one patient with a small insulinoma underwent exploratory laparoscopy first and then laparoscopic pancreas biopsy in a subsequent procedure. One patient underwent exploratory laparoscopy first and then laparoscopic enucleation in a second procedure due to intraoperatively detected pancreatitis. One patient underwent a laparoscopic attempt to resect a PNEN in the pancreatic tail, which required cconversion to laparotomy. In the same patient, a laparoscopic attempt at resection of a local recurrence also required conversion to laparotomy. 


\section{Paper II}

\section{Pancreatic surgery with vascular reconstruction in patients with locally}

advanced pancreatic neuroendocrine tumors.

J Gastrointest Surg. 2013 Jul; 17(7):1224-32.

This paper described seven patients with locally advanced PNEN who underwent pancreatic surgery with vascular reconstruction at a Norwegian university hospital. Four patients had metastatic disease at time of surgery. Four patients developed postoperative complications but there was no mortality associated with surgery. Median follow-up was 21 (3-58) months. One patient died 35 months after surgery, three patients had progressive disease 21, 9 and 4 months postoperatively, and three patients had disease in remission 58, 42 and 3 months postoperatively. 


\section{Paper III}

\section{Surgical treatment as a principle for patients with high-grade pancreatic}

neuroendocrine carcinoma: a Nordic multicenter comparative study. Ann Surg Oncol. 2016 May; 23(5):1721-8.

In this paper, the effect of surgery on oncological outcome in patients with PNECs was described in a Nordic multicenter patient cohort. One hundred and nineteen patients were included (Figure 13). Median time from surgery for nonmetastatic disease to development of metastasis was 7 months. The median survival was 23 months from time of metastasis for patients undergoing initial resection of the primary tumor in nonmetastatic disease (SURG1), 29 months for patients undergoing resection of the primary tumor and synchronous metastatic liver disease (SURG2), and 13 months for patients with synchronous metastatic disease receiving systemic chemotherapy only (CT2). The following factors were found to be statistically significant independent factors for improved survival after occurrence of metastatic disease: resection of primary tumor, $>4$ courses of chemotherapy, Ki67 $<55 \%$, and performance status 0 .

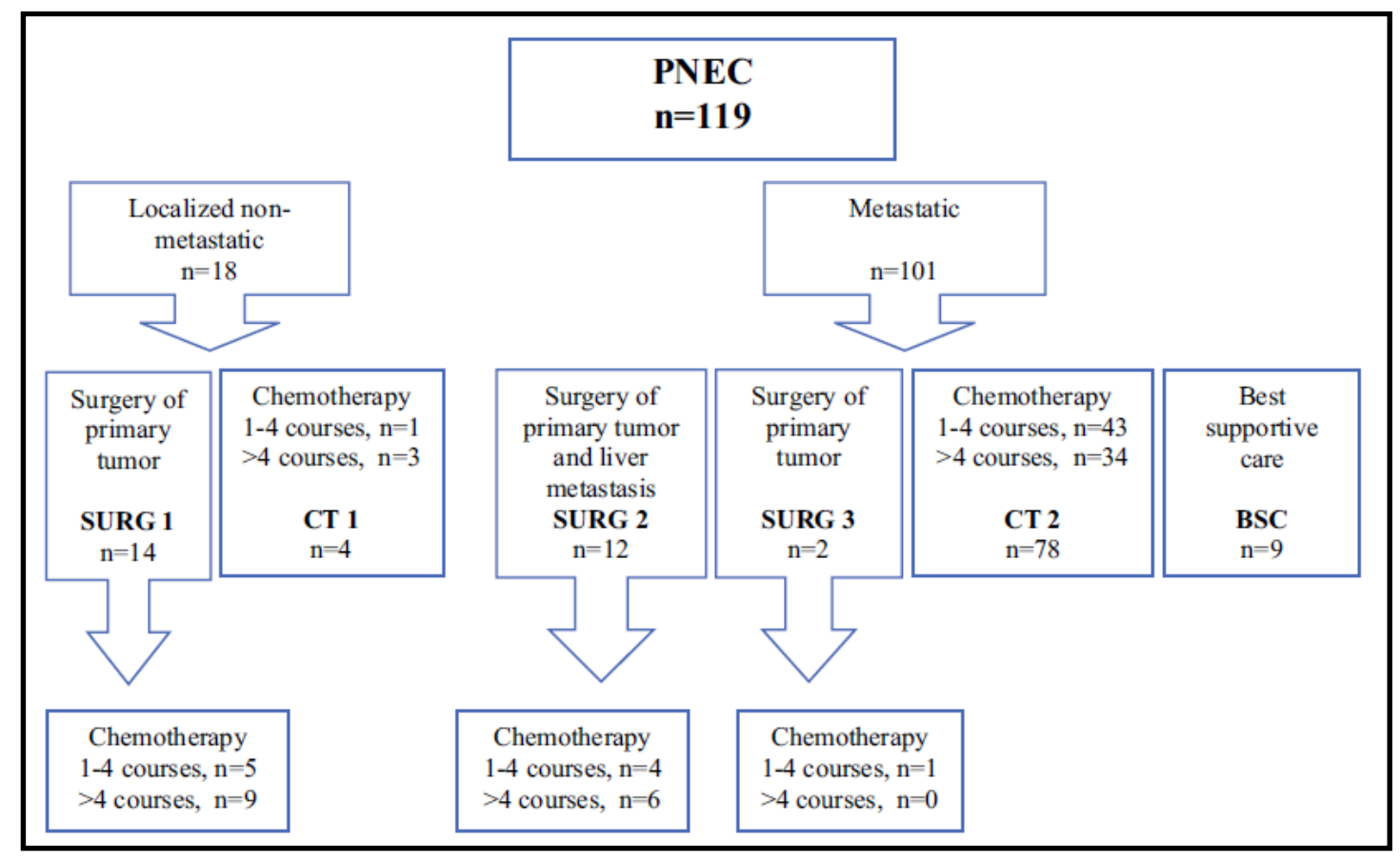

Figure 13. Flowchart of the patients and treatment groups in Paper III. Patient data on the number of chemotherapy courses were missing for three patients 


\section{Paper IV}

\section{Loss of 11p11 is a frequent and early event in sporadic nonfunctioning pancreatic neuroendocrine neoplasms.}

Oncol Rep. 2014 Sep; 32(3):906-12.

In this paper, screening of genomic imbalances in a series of 16 surgical specimens from 15 patients with sporadic PNEN was performed. G-band karyotyping and metaphase comparative genomic hybridization (CGH) were performed. G-banding revealed abnormal karyotypes in 2 of 10 tumor samples analyzed. DNA copy number changes were detected in 13 samples, whereas three tumors showed a balanced genome. In general, gains were more frequent than losses. Common gains were scored at 5p12-13, 4q13-24, 5p15, 5q11-31, and 9q21-22, whereas common losses were found at 11p11,11p14-15, 11q23, 11p12-13, and 11q22. The average number of copy aberrations (ANCA index) was 12 for 13 nonfunctioning primary tumors, 4.8 for the nonfunctioning tumors with low Ki67 ( $\geq 5 \%$ ), 21.2 for the tumors with high Ki67 $(<5 \%), 2.5$ for small tumors $(<3.5 \mathrm{~cm})$, and 17.8 for large tumors $(\geq 3.5 \mathrm{~cm})$. There was a statistically significant difference in the ANCA index between the groups defined by Ki67 and tumor size. Nonmetastatic nonfunctioning pancreatic neuroendocrine tumors with low Ki67 $(<5 \%)$ and small size $(<3.5 \mathrm{~cm})$ had few aberrations detected by $\mathrm{CGH}$, but frequent loss of material from chromosomal band $11 \mathrm{p} 11$. 


\section{Paper V}

\section{Transcriptomic profiling of tumor aggressiveness in sporadic}

nonfunctioning pancreatic neuroendocrine neoplasms.

Pancreas. 2016 Feb. Epub ahead of print.

This is an experimental study where high throughput RNA-seq was performed on eleven samples of sporadic nonfunctioning PNEN, grouped in mild disease $(\mathrm{n}=7$; Ki67 < 5\% and nonmetastatic disease) and aggressive disease ( $\mathrm{n}=4$; Ki67 $\geq 5 \%$ and metastatic disease), on Illumina's Genome Analyzer II platform. A set of 309 genes were statistically significantly differentially expressed between the two groups, out of which 143 were over- and 166 under-expressed in the aggressive disease group. Amongst the top protein-coding over-expressed genes, we found genes encoding proteins involved in DNA packaging (HIST1H2AL, $\log \mathrm{FC}=-4.1$, $\mathrm{P}$-adj=0.03; HISTIH2BF, $\log \mathrm{FC}=-3.8, \mathrm{P}$-adj=6.9e-04), chromosome structuring (TRIP13, $\log \mathrm{FC}=-3.7$; $\mathrm{P}$-adj=1.0e-06), cytoskeleton structuring $(A D D 2, \log \mathrm{FC}=-$ 3.5; P-adj=8.5e-04), cell-cell-signaling (WNT3, $\log \mathrm{FC}=-3.6 ; \mathrm{P}-\mathrm{adj}=1.7 \mathrm{e}-08$; ITPKA, $\log \mathrm{FC}=-3.6 ; \mathrm{P}-\mathrm{adj}=5.9 \mathrm{e}-06)$, and ability to taste $(T A S 2 R 38, \log \mathrm{FC}=-3.7$; $\mathrm{P}$-adj=0.03). Amongst the top protein-coding under-expressed genes, we found genes encoding proteins involved in neuronal differentiation $(M Y T 1 L, \log \mathrm{FC}=5.1$; $\mathrm{P}$-adj=8.9e-09), cytoskeleton structuring (KRT27, $\log \mathrm{FC}=3.8 ; \mathrm{P}-\mathrm{adj}=2.1 \mathrm{e}-03)$, cell-cell-signaling $(G A B R P, \log \mathrm{FC}=3.8 ; \mathrm{P}-\mathrm{adj}=2.2 \mathrm{e}-03)$, and the immune system (CTSE, $\log F C=3.7 ; \mathrm{P}-\mathrm{adj}=0.003$ ). 


\section{Methodological considerations}

\section{Patient selection and ethics}

The patients included in the studies of this thesis underwent treatment for PNEN in the period between 1997 and 2013. Patients included in Paper I (n=72), II $(\mathrm{n}=7)$, IV $(\mathrm{n}=15)$, and $\mathbf{V}(\mathrm{n}=11)$ all underwent surgery at the Department of Hepato-Pancreato-Biliary Surgery at Oslo University Hospital, Oslo, Norway. Patients included in Paper III ( $n=119)$ underwent treatment for PNEC at one of the following Nordic university hospitals: Oslo University Hospital ( $\mathrm{n}=14 ; 7$ with surgery), Uppsala University Hospital (Sweden, $\mathrm{n}=28 ; 4$ with surgery), Copenhagen University Hospital (Denmark, $n=25$; 1 with surgery), Karolinska University Hospital (Sweden, n=2; no surgery), Helsinki University Hospital (Finland, n=13; 8 with surgery), Haukeland University Hospital (Norway, n=10; 3 with surgery), Trondheim University Hospital (Norway, n=2; no surgery), Stavanger University Hospital (Norway, n=3; no surgery), Aarhus University Hospital (Denmark, n=12; 2 with surgery), and Odense University Hospital (Denmark, $\mathrm{n}=10 ; 3$ with surgery).

As PNENs are rare and clinically diverse, prospective studies on homogenous cohorts of patients with PNENs are hard to conduct. This is reflected by the fact that there are as yet no published randomized controlled trials involving surgery in this group of patients. The clinical studies contained in this thesis were of retrospective design. This may have led to missed cases of relevant PNEN patients in the study period. The Department of Hepato-Pancreato-Biliary Surgery, Oslo University Hospital, was the only institution performing laparoscopic pancreatic surgery and pancreatic surgery with vascular reconstruction in the South-Eastern Norway Regional Health Authority in the study period. This health authority serves about 2.7 million, is the nation's largest, and includes more than half of Norway's inhabitants. The patient cohorts in Papers I and II should sufficiently represent the corresponding health region in the period 1997-2011 for Paper I, and 2007-2012 for Paper II. Due to the highly aggressive nature and low incidence of PNECs, one could assume that many patients with PNEC may have died before 
being diagnosed or referred to a university hospital. This represents an important selection bias in Paper III. Because the data were acquired from several institutions in different countries, there may also have been a selection bias associated with divergent diagnostic and treatment strategies.

There was no overlap among the patient cohorts of Papers I, II and III. Tumor tissue obtained from one patient in Paper I was used in Papers IV and V, among tissue samples from other patients. Likewise, tumor tissue obtained from one patient in Paper II was used in Papers IV and V, among tissues from other patients. Tumor tissues obtained from 11 patients in Paper IV were used in Paper V. In total, findings from 213 unique patients with PNEN were included in this thesis.

Tissue samples examined in the studies of Papers IV and $\mathbf{V}$ were collected from the Institutional Biobank for neuroendocrine neoplasms at Oslo University Hospital, established in 2011. In Paper IV, patients with sporadic nonfunctioning PNENs were divided in groups according to the Ki67 index of the primary tumor, size of the primary tumor, and whether or not metastatic disease was present at time of surgery. Intertumor copy number variation between the groups, quantified by $\mathbf{C G H}$, was compared. In Paper $\mathbf{V}$, the intertumor variation of transcripts of protein-coding genes, i.e., differential expression, was described by means of highthroughput RNA-seq of tissue samples from sporadic nonfunctioning PNENs. Tumor samples were compared according to "aggressive" or "mild" tumor behavior, defined by the primary tumor's Ki67 index and patient's metastatic status. Genetic screening for familial neuroendocrine syndromes was not performed routinely upon diagnosis of a PNEN among the patients included in this thesis. There may therefore have been cases of unrecognized familial PNEN among the patients included.

Papers I and II are classified as clinical audits and necessary permissions were obtained from the hospital review board. Papers III, IV and V are classified as 
research and were approved by the Regional Committee for Medical and Health Research Ethics (project number: 2012/490 and 2011/1945D), respecting the Helsinki Declaration ${ }^{155}$. The Biobank for neuroendocrine neoplasms at Oslo University Hospital is approved by the Regional Committee for Medical and Health Research Ethics (project number: 2011/497A).

In Papers I and II, the revised Accordion Classification was used for definition of surgical morbidity ${ }^{156}$ and the International Study Group Definition of Pancreatic Fistula (ISGPF) was used for definition of $\mathrm{POPF}^{157}$.

In Paper V, PNEN tissue was among other variables categorized according to the Ki67 index. Of the 11 samples examined, seven had a Ki67 index of 1-2\% while the other four had a $\mathrm{Ki} 67$ index $\geq 12 \%$. We believe this was a good design in the sense that there was not an intermediate range of $\mathrm{Ki} 67$ values. The potential for identifying differentially expressed genes based on the Ki67 index was thus maximized.

\section{Statistical analysis}

In Paper I, continuous data were presented as median (range) and analyzed using the non-parametric Kruskal-Wallis test for independent samples. Median was chosen over mean in order to minimize unwanted effects of extreme outliers in the relatively small patient cohorts. A normal distribution was not assumed, as the low sample size in each group did not necessarily indicate such distribution. The Kruskal-Wallis test was applied in order to compare group differences in four independent groups for both continuous data (age, body mass index (BMI), operative time, intraoperative bleeding, and hospital stay) and nominal data (surgical morbidity and POPF). In retrospect, the Chi-squared test should have been used instead of the Kruskal-Wallis test to compare nominal data in Paper I. This has later been done and results in a statistically significant group difference for POPF ( $\mathrm{p}=0.029)$, but no statistically significant group difference for overall 
surgical morbidity $(\mathrm{p}=0.439)$, which is consonant with the results already presented and discussed in Paper I.

In Paper I, post-hoc analysis with Bonferroni correction of multiple comparisons and Tukey's test were suggested following rejection of the Kruskal-Wallis test. As the Kruskal-Wallis test was only rejected in one case of group comparisons of nominal data (POPF), such post-hoc analysis was not possible to perform. Instead, a post-hoc analysis of the Chi-squared test results for POPF could have been performed in this case. This has later been done with contingency table analysis, as described by Beasley et al. ${ }^{158}$. First, a contingency table analysis was performed on the chi-square analysis. Then, adjusted standardized residuals (Z-values) for each cell were calculated before they were transformed to chi square values and then to p-values. Finally, the p-values were compared against the Bonferronicorrected $\mathrm{p}$-value. This resulted in a statistically significant correlation between laparoscopic enucleation of PNENs in the pancreatic head and the development of POPF $(\mathrm{p}=0.015)$. This conclusion was not included in the published paper.

One weakness of the statistical model used in Paper I was the suggested use of a post hoc test (Tukey's test) meant for parametric data on the assumption of nonparametric data. A more appropriate test would be the Mann-Whitney test for group comparisons after correction for multiple comparisons by the Bonferroni method or Dunn's test. As no statistically significant group differences for continuous data were found with the Kruskal-Wallis test in Paper I, there was no need to run an adjusted post-hoc analysis. In Paper I, disease-specific survival was estimated using Kaplan-Meier curves and the log-rank test was used to compare differences in survival among patient subgroups.

Only descriptive statistics was performed in Paper II due to the low sample size (7 patients). 
In Paper III, descriptive statistics were presented as frequencies, medians, ranges, and proportions. Overall survival was constructed using Kaplan-Meier curves with accompanying risk tables. Cox-proportional hazard models (uni- and multivariate) were fitted for evaluation of the effect of factors potentially influencing survival. Due to the limited number of patients included in Paper III ( $\mathrm{n}=119)$, a statistical model with five variables was constructed. Each of the chosen variables was tested for clinical relevance (resection of primary tumor, courses of chemotherapy, Ki67 index, small cell morphology, and performance status (PS)) and independence before they were included in the Cox-analysis. The statistical analysis included in Paper III was planned and performed in close cooperation with a statistician.

In Paper IV, the ANCA index was used to define the prevalence of genomic imbalances in each tissue group. The Mann-Whitney U test was used to compare median for two independent samples without the assumption of a normal distribution.

In Paper V, we applied the DESeq2 for differential gene expression analysis of RNA-seq data, using the Wald test ${ }^{159}$. The selected method uses shrinkage estimation for dispersions and fold changes (FCs) to improve stability and interpretability of the estimates. Functional annotation analysis was performed using the Database for Annotation, Visualization and Integrated Discovery $(\mathrm{DAVID})^{160}$, which allowed identification of overrepresented functional categories among the genes that were differentially expressed.

\section{Pathology}

Preoperative cytology or biopsy was generally not performed in the patients included in this thesis. For the patients operated at Oslo University Hospital, preoperative percutaneous biopsy of PNENs was generally avoided because of the theoretical risk of tumor dissemination ${ }^{161}$, despite limited evidence for the occurrence of this phenomenon in the case of PNENs. In some cases, preoperative endoscopic ultrasound-guided fine needle aspiration cytology was performed. As 
the presence of PNENs is largely detected by cross-sectional (CT and MRI) and nuclear imaging, exact grading by quantification of the mitotic rate and/or Ki67 index is typically possible only after tissue sampling. The possibility of preoperative evaluation of grading of PNENs is a matter of debate, as current techniques for tissue sampling have limitations ${ }^{162}$. The lack of information on tumor grading preoperatively may have influenced the surgeon's choice of procedure and therefore represents a bias.

All surgical specimens associated with Papers I, II, IV and V were assessed by pathologists at the Department of Pathology, Oslo University Hospital. During the study period of Paper I, the WHO Classification for NENs changed ${ }^{31,163}$. The Ki67 index then became essential for classification purposes. PNENs assessed before the introduction of the current WHO 2010 Classification without quantification of the $\mathrm{Ki} 67$ index were re-assessed in order to allow for reclassification. The remaining specimens were not re-assessed. A re-evaluation of all surgical specimens by two or three independent pathologists would have improved validity of histopathological data in all papers of this thesis. In Paper I, a resection status of $\mathrm{R} 2$ was defined as residual metastatic disease and not residual local disease.

Paper I revealed a relatively high fraction of surgical specimens with an Nx status, indicating that lymph nodes were not found by the pathologist. Most of the patients included in Paper I underwent distal pancreatectomy, with or without concomitant splenectomy. The total number of lymph nodes found in three of the seven patients included in Paper II was remarkably low with two nodes found in a Whipple specimen, one node found in a distal pancreatectomy specimen, and no lymph nodes found in another distal pancreatectomy specimen. This raises questions about suboptimal surgical technique or issues related to suboptimal pathological assessment of the distal pancreatectomy specimens. In the study period of this thesis, pathology assistants performed the gross examination of pancreas specimens at our institution. Peripancreatic lymph nodes were routinely searched 
for by palpation and sight, and, if found, dissected from the main specimen with surrounding adipose tissue and sent for histological assessment. Thus, small lymph nodes could have been overlooked. Suboptimal lymph node sampling in PNEN specimens has been reported by others ${ }^{164}$. The method of gross examination of the PNEN specimens at our institution may represent a bias toward underreporting of the actual number of peripancreatic lymph nodes present. One measure of improvement could be to identify, dissect and embed standardized peripancreatic lymph node regions regardless of macroscopic findings.

As PNECs are morphologically and biologically heterogeneous ${ }^{165}$, thorough and standardized histopathological reporting is of great importance for treatment planning and prognostic evaluation of patients. Due to the low incidence of PNEC and the ensuing risk of misdiagnosis, cases should be reviewed by pathologists with expertise in the evaluation of GEP-NENs ${ }^{112}$. In Paper III, pathologists at each of the participating ten institutions assessed surgical specimens and biopsies. However, there was a lack of a centralized pathology re-evaluation. This is one of the major weaknesses of the study, as it is known that well differentiated neuroendocrine neoplasms and acinar cell carcinoma can be misdiagnosed as $\mathrm{PNECs}^{112}$.

\section{Karyotyping and comparative genomic hybridization (CGH)}

In Paper IV, karyotyping and metaphase CGH were performed on samples of PNEN. The aim of the study was to compare intertumor copy number variation between different groups of samples. Tumor samples were disaggregated mechanically and enzymatically and the resulting cells and cell clumps were cultured for 7-10 days. Abnormal karyotypes were only present in two of 10 analyzed tumor samples. As karyotyping of tumors requires culturing of neoplastic parenchyma cells in vitro, the low yield of abnormal karyotypes could indicate poor division of neoplastic parenchymal cells in the cell cultures. In our study, we applied a standardized cell culture protocol previously used in our lab with satisfactory results for solid neoplasms ${ }^{166}$. Systematic measures to modify the cell 
culture medium and protocol during the study period were not undertaken. It appeared to us that pancreatic neuroendocrine tumor cells do not divide well under laboratory conditions. This may account for the severely limited cytogenetic information of PNENs hitherto reported in the literature with only seven karyotypical abnormal cases in three studies ${ }^{140-142}$. The difficulty in growing pancreatic neuroendocrine cells may be explained by the fact that most PNENs are highly differentiated with a relatively low proliferating rate in vivo, as was the case for eight of the 15 patients (Ki67 index < 5\%) in our study. Other possible reasons for the low number of clonal aberrations detected by karyotyping could be overgrowth of stromal fibroblasts or contamination with bacteria or yeast, which are known threats to cultures of neuroendocrine cells ${ }^{167}$. When comparing the cell culture protocol applied in our study with protocols applied in studies of successful culturing of pancreatic neuroendocrine cells ${ }^{168-170}$, no clear reasons for our failure in establishing effective cell cultures appeared. One measure of improvement in our study could be to implement control of the purity of the neuroendocrine cell preparations. This could have been done by immunostaining with antibodies against specific neuroendocrine cell antigens, such as chromogranin $\mathrm{A}$ and synaptophysin ${ }^{168}$. When cytogenetic analysis is performed on cultured cells, it is important to consider whether the results are representative of the in vivo situation $^{121}$. Two main types of heterogeneity can be seen in cytogenetic analysis of tumor samples: heterogeneity between neoplastic and non-neoplastic cells, and heterogeneity among various neoplastic cells ${ }^{171}$. Short-term cultures, such as in our study, help minimize such heterogeneity and should therefore be preferred.

In Paper IV, CGH was performed on isolated DNA from representative freshfrozen PNEN tissue. The presence of neuroendocrine tissue in each specimen used in Papers IV and V was confirmed by histopathology. Whereas metaphase CGH allows investigation from a chromosomal band level under the microscope, array $\mathrm{CGH}$ allows investigation of individual genes ${ }^{172}$. As mentioned above, one limitation of both $\mathrm{CGH}$ techniques is that they reflect a theoretical average of tumor samples so that intercellular variability is impossible to assess ${ }^{121}$. Another 
limitation is the failure to detect balanced rearrangements such as inversions, insertions, and translocations. As the aim of Paper IV was to screen the tumor genome of PNENs for genetic imbalances, without identification of individual genes, metaphase $\mathrm{CGH}$ seemed appropriate.

\section{RNA sequencing and bioinformatics approach}

In Paper V, high-throughput RNA-seq was performed on PNEN tissue and the resulting sequencing data were further analyzed with bioinformatics tools. RNAseq was performed on isolated RNA from representative fresh-frozen PNEN samples stored at $-80^{\circ} \mathrm{C}$. Before sequencing, the RNA quality of all tumor samples was evaluated. Insufficient RNA quality was found in four samples out of 15 primary PNENs and these were excluded from further analysis. High-throughput paired-end RNA-seq was performed on a HiSeq 2500 (Illumina, San Diego, CA, USA) platform at the Norwegian Sequencing Centre in Oslo, Norway. Validation of the findings with gene expression profiles of other PNEN series or public datasets was unsuccessful in that $\mathrm{Ki} 67$ and clinical data were inconsistently defined in such series, and were not identified in gene expression omnibus and similar databases. This is an important limitation of this study. The lack of validation of the RNA-seq results with other methods, such as real-time PCR or immunohistochemistry in the same patient cohort or larger independent cohorts of sporadic nonfunctioning PNEN, is another important limitation of this study. 


\section{General discussion}

In this thesis, we sought to investigate different aspects of modern surgical treatment for PNENs (Papers I, II and III). Based on surgical specimens from sporadic nonfunctioning PNENs, we then wanted to identify gene expression patterns that may be important for molecular differentiation of tumor aggressiveness (Papers IV and V). Below follows a discussion of the main findings.

\section{Laparoscopic surgery for PNENs}

At the beginning of the study period for this thesis, there were only few published large series describing laparoscopic surgery of PNENs, with cohorts of less than 50 patients ${ }^{82,83}$. These studies did not include information on grading according to the current WHO 2010 classification or information on long-term survival related to the laparoscopic approach alone.

In Paper I, we reported the, at the time of publication, largest single center series of patients undergoing laparoscopic surgery for PNENs. In a cohort of 72 patients, we demonstrated that laparoscopic surgery with enucleation or distal pancreatectomy, with or without splenectomy, is feasible in patients with PNENs. Feasibility was based on tumor size, operative time, and hospital stay. The operated tumors had a relatively small median diameter of $2.2 \mathrm{~cm}$, but even smaller $(0.5 \mathrm{~cm})$ and much larger lesions $(9.5 \mathrm{~cm})$ were successfully removed. Whereas laparoscopic distal pancreatectomy (LDP) was performed on both small and large tumors, laparoscopic enucleation (LE) was only performed on relatively small lesions $(0.8-2.8 \mathrm{~cm})$. Our findings indicate that LDP is not limited by the size of the primary tumor in patients with PNENs, which had been shown previously and also been confirmed in similar studies, where resections of PNENs with diameters up to $13 \mathrm{~cm}$ had been reported ${ }^{83,173}$. The median operative time for LDP in our study ranged from 175 to 190 minutes, depending on whether concomitant splenectomy was performed or not (shorter with splenectomy). This is similar to earlier reported operative times for LDP $(157-230 \mathrm{~min})^{72,73,174}$ and open distal 
pancreatectomy (130-216 $\mathrm{min})^{73,175}$ with and without concomitant splenectomy. The marginally longer operative time for LDP, compared with open distal pancreatectomy, could be explained by inclusion of cases performed when surgeons were still on the early learning curve for the laparoscopic approach. This is supported by evidence of shorter operative times when experience with LDP increases ${ }^{174}$. The duration of LE in our study was shorter than for LDP, especially for PNENs in the pancreatic body or tail (head, $167 \mathrm{~min}$ versus body/tail, $111 \mathrm{~min}$ ). This is coherent with the operative time for $\operatorname{LE~}(120 \mathrm{~min})^{72,176}$ and for open enucleation (140-162 $\mathrm{min})^{175,177}$ reported by others. Operative times do not seem to differ significantly between laparoscopic and open distal pancreatectomy/enucleation in patients undergoing such surgery for PNENs.

Patients undergoing pancreatic surgery are generally at high risk for development of postoperative morbidity and require optimal surgical and postoperative care. However, as surgical techniques and perioperative monitoring and management have improved, durations of hospital stays have become shorter. One important advantage of laparoscopic pancreatic surgery is reduced hospital stay compared with open pancreatic surgery ${ }^{178}$. The median hospital stay in our study was 7 days, which is similar to observations in other studies of LDP and/or LE (6-11 days $)^{73}$, 179 and open distal pancreatectomy and/or pancreatic enucleation (8-9 days) ${ }^{175}$. In conclusion, Paper I shows that laparoscopic pancreatic surgery, exemplified by LDP and LE, is feasible in the treatment of PNENs. These findings are in agreement with results of comparable studies ${ }^{67,69-71}$.

As mentioned above, pancreatic surgery is generally associated with a high risk of postoperative morbidity. In particular, development of pancreatic fistula is a feared complication besides other common morbidities such as septic complications following intra-abdominal abscess formation and abdominal hemorrhage. Developments in pancreatic surgical techniques aim at minimizing the risk of morbidity and mortality, and improvements have been noted throughout the last three decades ${ }^{180}$. 
In Paper I, we found an overall postoperative complication rate of about $42 \%$ with no surgery-related mortality in patients undergoing LDP and LE for PNENs. This is comparable to overall complication rates in similar studies of patients undergoing pancreatic enucleation and resection by laparotomy $(45 \%)^{181}$ and previous studies of LDP and LE for PNENs (31\%) $)^{83}$. In our study, POPF was the most frequent overall complication and also the most frequent severe complication, occurring in about $21 \%$ of the patients. This observation is also in accordance with previously described complication rates in comparable laparoscopic procedures $(22 \%)^{83}$. Other complications occurred primarily after LPD. These were mild or moderate, such as transient fever treated successfully with antibiotics or a temporary fall in Hemoglobin, which was managed by blood transfusion. An interesting and important finding in our study was the relatively high rate of POPF after LE compared with LDP (50\% versus 14\%). A higher rate of POPF after enucleation has also been observed in open surgery when compared with resection $(38 \% \text { versus } 15 \%)^{181}$. This might indicate a higher risk of intraoperatively unrecognized damage to the main pancreatic duct when performing enucleations. Hence, preoperative planning with identification of the main pancreatic duct and estimation of the distance to the tumor margin on crosssectional imaging is essential to minimize intraoperative damage to the duct and subsequent pancreatic leakage. Based on the high risk of POPF after LE compared with LDP observed in our study, we now routinely perform LDP for PNENs located in the pancreatic body and tail.

Whereas LDP represents a feasible minimally-invasive alternative to LE for PNENs in the pancreatic body and tail, the surgical alternatives to enucleation for removal of lesions located in the pancreatic head are more invasive (e.g., pancreatico-duodenectomy or duodenum-preserving pancreatic head resection ${ }^{182}$ ). Open enucleation for patients with PNENs in the pancreatic head has been associated with decreased blood loss, shorter operative time, and shorter hospital stay compared to open pancreatico-duodenectomy ${ }^{175,181,183}$. Based on this, we 
recommend that noninvasive and small PNENs in the pancreatic head be considered for LE rather than more invasive procedures.

In conclusion, Paper I demonstrates that feasibility and postoperative morbidity is acceptable after LDP for PNENs in the pancreatic body and tail and acceptable after LE for PNENs in the pancreatic head. The latter conclusion is supported by other studies which have shown that enucleation of pancreatic neoplasms is associated with long-term survival, despite a relatively high risk of pancreatic fistula formation ${ }^{77,} 177$. However, bearing the high risk of POPF and associated complications in mind, we believe that decisions regarding LE for lesions located in the pancreatic head should be evaluated for each individual patient at hepatopancreato-biliary centers with experience in advanced laparoscopy.

The direct comparison of the results of this study with the findings in other studies should be done with caution as different classification systems for surgical morbidity exist and are not uniformly applied. An obvious weakness of our study is the retrospective design. Thirty-day postoperative morbidity was not assessed systematically for all patients, and occurrence of complications after discharge may therefore have been underreported in our study. Another limitation is that data on postoperative exocrine and endocrine insufficiency were not included. This could potentially have given additional information about the benefits of parenchyma-sparing procedures as the development of exocrine and endocrine insufficiency in theory should be higher in resections compared with enucleations $^{183}$.

In Paper I, we also evaluated the prognostic value of the WHO 2010 grading system $^{31}$ and showed that the suggested cut-off between a PNET G1 and a PNET G2 tumor, defined by a Ki67 index of $2 \%$, did not predict survival in a cohort of patients undergoing laparoscopic surgery for PNENs. Based on similar observations by others, we then tested a Ki67 cut-off value of 5\% and could show that this was a significant predictor of prognosis. This is coherent with the results 
of comparable studies ${ }^{38,43,44,85,108}$ and may indicate the need for a change in the grading classification for PNET G1 and PNET G2 in the WHO system.

In our study, we also demonstrated a favorable oncological long-term outcome in patients undergoing laparoscopic surgery for PNENs, with an overall 5-year survival of $90 \%$. Based on the Kaplan-Meier estimates, prognosis seemed to correlate with the size of the primary tumor (T stage), something that has been shown previously ${ }^{38,}{ }^{184}$. The excellent long-term prognosis after surgery for PNENs in our study, with lesions with a median diameter of $2.2 \mathrm{~cm}$, may question the liberal use of surgery for small indolent nonfunctioning PNENs as pancreatic surgery in general is associated with high risk of postoperative morbidity. There is an ongoing discussion about whether small indolent PNENs should be observed or operated. In our study, nonfunctioning PNENs with a diameter as small as $0.5 \mathrm{~cm}$ underwent surgery. This is in contrast to other reports where indolent nonfunctioning PNENs $<2 \mathrm{~cm}$ generally seem to be observed rather than removed $^{15,26,83}$. In our department, the general opinion has been that surgery should be attempted in any case of a suspected localized PNEN as long as the patient's PF is satisfactory. This is supported by the fact that even small PNETs $<$ $2 \mathrm{~cm}$ can metastasize, and cases of small PNECs do occur, as exemplified by the patient cohort in Paper III of this thesis and other studies ${ }^{185,186 .}$

In Paper I, histopathology showed that most patients undergoing a resection or enucleation of a PNEN, were classified as ENETS T1 (47\%), Nx (69\%), and G1 $(69 \%)$. Most of the patients did not have metastatic disease at time of surgery $(83 \%)$. An unexpected finding in our study was the high number of specimens without any observed lymph nodes (Nx status) while noticing a positive lymph node status in a third of the patients where lymph nodes were actually found. In specimens after both open and laparoscopic distal pancreatectomy, an average lymph node sampling rate of 12-14 has been reported, with no significant difference between open and laparoscopic surgery ${ }^{76}$. However, there are also reports showing a lower sampling rate in LDP compared with open surgery ${ }^{74}$. On 
the other hand, enucleations are associated with a low lymph node sampling rate compared with standard resections (such as distal pancreatectomy) ${ }^{164}$. An important observation in our study, which was not addressed in the published paper, was that nearly all spleen-preserving LDP specimens were classified as $\mathrm{Nx}$ (22 of 23 specimens), whereas this was the case in only a third of the LDP specimens with spleen (9 of 28 specimens). Hence, LDP with concomitant splenectomy seems to increase the lymph node sampling rate in PNEN specimens. Furthermore, all of the $14 \mathrm{LE}$ specimens were classified as Nx. The high number of specimens with an Nx status in our study raises questions about suboptimal surgical technique, as it is known that surgical technique in patients undergoing distal pancreatectomy influences the lymph node sampling rate ${ }^{187}$. Low lymph node yield after distal pancreatectomy has led to development of techniques such as the radical antegrade modular pancreatosplenectomy (RAMPS) ${ }^{187,}{ }^{188}$. This procedure is also possible to perform laparoscopically ${ }^{189}$. We have not found obvious surgical reasons for the generally low lymph node yield in our study. Therefore, in cooperation with the pathologists at our institution, we have now initiated a protocol for standardized pathological assessment of LDP specimens in order to find ways of improving the lymph node sampling rate.

The role of lymph node sampling in PNEN is not yet fully understood, but there are several studies suggesting lymph node status as a prognostic factor ${ }^{44,190-196}$. It is also known that the risk of peripancreatic lymph node metastasis in patients with PNENs correlates with increasing tumor size and tumor grading ${ }^{193}$. However, only about $40 \%$ of patients with PNENs who undergo surgery have lymph node metastasis ${ }^{26}$, which could indicate that lymphadenectomy should not be performed in all patients. Thus, there is an ongoing debate as to whether or not peripancreatic lymphadenectomy should be performed routinely in patients with PNEN, regardless of the type of surgical procedure ${ }^{190-192,197-199}$.

It is of great importance to know the prognostic implications of positive lymph nodes in patients with PNENs and to know to what extent laparoscopic pancreatic 
surgery can provide sufficient lymph node sampling to achieve optimal oncological outcome. Taking recent observations from other studies into consideration and knowing that issues related to lymph node sampling in patients with PNENs have not been investigated in a randomized manner, we believe that lymph node sampling should be performed routinely when performing laparoscopic removal of PNENs, to avoid understaging ${ }^{78,200}$.

Since the initiation of the work contained in this thesis, LDP has now become an established procedure at several institutions worldwide ${ }^{72,73,75,79,174,178,201-203}$. The procedure provides similar short- and long-term oncological outcomes as open distal pancreatectomy ${ }^{76}$ and seems to be a cost-efficient alternative to open distal pancreatectomy ${ }^{80}$. Besides LDP for PNENs in the pancreatic body and tail, laparoscopic enucleation of nonfunctioning PNENs in the pancreatic head ${ }^{204}$ and laparoscopic pylorus-preserving pancreatico-duodenectomy ${ }^{205}$ (laparoscopically assisted or total laparoscopic) are advanced, but feasible procedures that can be considered in selected cases. A single-incision approach could also be performed, with comparable feasibility measures ${ }^{206}$. Attempts to compare laparoscopic and open surgery for PNENs have been made, but the results are inconclusive since different procedures are included ${ }^{82,207}$. The first comparative study of laparoscopic and open distal pancreatectomy for patients with PNENs was recently published ${ }^{173}$ showing a significant reduction in postoperative morbidity without compromising oncological outcomes and survival. In our department, initiatives have been taken to conduct a retrospective international multicenter comparative study between laparoscopic and open distal pancreatectomy.

In conclusion, Paper I demonstrates that laparoscopic surgery of PNENs is feasible with acceptable surgical morbidity and a good overall disease-specific long-term prognosis. This is supported by the current ENETS consensus guidelines, where Paper I has been cited ${ }^{15}$. However, laparoscopic surgery for PNENs is still a barely investigated field of surgery. Prospective surgical trials are difficult to conduct due to the low incidence and significant clinical heterogeneity 
of the disease. This is reflected by the fact that there are no trials on surgery for PNEN registered in ClinicalTrials.gov ${ }^{208}$ (a registry of clinical studies maintained by the American National Library of Medicine). As the number of comparative studies on laparoscopic surgery for PNENs is very limited and no randomized trials exist to date, surgeons should focus on conducting large retrospective multicenter comparative studies and consider initiating randomized trials ${ }^{209}$.

\section{Vascular reconstruction in patients with PNENs}

The increasing use of laparoscopic pancreatic surgery in the treatment of PNENs in recent years could indicate a parallel gradual decline in the use of open pancreatic surgery for this group of patients. However, we have observed that as many PNENs are nonfunctioning and slow-growing, a substantial proportion of these present with locally advanced disease, which may not be tackled by minimally-invasive procedures. In this thesis, locally advanced disease was defined as a PNEN with an ENETS T3- (confined to pancreas, $>4 \mathrm{~cm}$, or invasion of duodenum or bile duct) or T4-stage (invasion of adjacent organs or major vessels).

Surgical treatment of locally advanced PNENs, and T4 tumors in particular, remains controversial ${ }^{84}$. There are previous reports which discuss vascular reconstruction among patients with locally advanced PNENs ${ }^{84,86-96,210}$, but none of these discuss the role of vascular reconstruction as such. This is remarkable, as the concept of vascular reconstruction has already been discussed widely over years in the treatment of locally advanced PDAC $97,98,211$, generally a much more aggressive disease. Among patients with PDAC, combined portal vein resection and reconstruction provides acceptable morbidity and mortality, compared with nonsurgical treatment ${ }^{101}$. Therefore, in Paper II, we wanted to evaluate the feasibility and surgical morbidity of pancreatic surgery in all patients with locally advanced PNEN who underwent pancreatic surgery with vascular reconstruction in the period between 2007 and 2012. The study is among the largest addressing the issue of pancreatic resection with vascular reconstruction for locally advanced 
PNENs and shows that this is feasible with acceptable postoperative morbidity and no mortality.

In Paper II, we reported vascular reconstruction in seven patients with locally advanced PNEN. Vascular reconstruction was performed on the portal vein, common hepatic artery, left hepatic artery, and/or left gastric artery. In addition to vascular surgery, extensive visceral resections were required in five patients. Relatively high levels of intraoperative bleeding (500-4750 ml) and long operative times (232-718) were observed. Severe complications developed in two patients, with upper gastrointestinal bleeding secondary to ischemic necrosis of parts of the stomach in one patient (successfully treated with transfusion) and development of a liver abscess and pseudoaneurysm of the pancreatico-duodenal artery (successfully treated with percutaneous transluminal embolization). The complexity of the procedures led to a relatively long overall median hospital stay (median 25 days).

Previous studies have shown that resection for locally advanced PNENs is feasible and can result in favorable disease-free survival and overall survival in selected patients $^{212}$, despite risk of recurrence ${ }^{84}$. This is supported by evidence that resection of the primary tumor in patients with PNEN is associated with improved survival across all stages of disease ${ }^{55}$. When not operated on, patients with locally advanced disease may suffer from complications related to local mass effect and infiltrative growth, including gastrointestinal bleeding, vascular/intestinal/biliary obstruction, and occlusion of the superior mesenteric (SMV) or portal vein $(\mathrm{PV})^{48}$, 86.

Today, surgery for locally advanced PNENs with vascular involvement remains controversial, which is probably reflected by the low number of studies published on this topic. Our study has certain limitations besides the obviously low number of samples. It is of retrospective design and includes some patients who underwent concomitant resection of metastatic liver disease. This may have biased feasibility 
and surgical morbidity. However, taken these limitations into consideration and based on the findings in our study, which are coherent with findings from previous studies on locally advanced PNENs, we would recommend that surgery is always considered in these patients, even in cases where vascular encasement or infiltration is suspected. This is also in line with the current ENETS consensus guidelines, where Paper II has been included as one of the references ${ }^{15}$. Important to remember is that in most cases where preoperative radiologic evaluation suggests vascular involvement, or the intraoperative findings of a partial encasement of a large vessel, the tumor can be removed with careful dissection without the need of vascular reconstruction ${ }^{91}$.

\section{Surgery for PNECs}

PNEC is a highly malignant disease that typically invades adjacent structures or has metastasized at diagnosis ${ }^{213}$. At the beginning of the study period for this thesis, some surgeons ${ }^{120}$, oncologists ${ }^{113}$ and pathologists ${ }^{45}$ had shown interest in this rare and highly aggressive malignancy. The largest cohort of patients with advanced GEP-NECs was published by the Nordic Neuroendocrine Tumor Group $(\mathrm{NNTG})^{113}$ in 2013. The cohort included 305 patients, out of whom 71 had a PNEC, which was associated with a median survival of only 15 months. Interestingly, $15 \%(n=11)$ of the PNEC patients had a resection of the primary tumor performed. However, the role of surgery was not assessed further. Surgical resection is an established treatment method for $\mathrm{PDAC}^{214}$, a much more common cancer than PNEC with an incidence ratio of $11-14$ per 100,000 person-years ${ }^{215}$, 216 (versus < 0.1 per 100,000 person-years for PNECs), but with comparable malignant potential. As improvement of treatment options and treatment sequences in patients with PNEC is urgently needed, we wanted to investigate the effect of surgery on survival and to identify potential prognostic factors for the survival in patients with metastatic PNEC.

In Paper III, we described the first comparative study on the effect of combined surgical treatment and chemotherapy versus chemotherapy alone, in patients with 
PNEC. In a cohort of 119 patients, 28 patients underwent combined surgery, 82 patients received only chemotherapy, whereas 9 patients received best supportive care. During a median follow-up period of 13 months (0-165 months), 92 patients (77\%) died of disease. In this study, we showed, for the first time, that surgical treatment combined with chemotherapy may improve survival in patients with metastatic PNEC compared with chemotherapy alone.

One of the main findings in our study is that resection of the primary tumor was an independent prognostic factor of improved survival for patients at different disease stages (HR 1.28, 95\% CI 1.4-5.7). The study demonstrated that surgery and chemotherapy of nonmetastatic disease improved survival, despite recurrent disease after a median of 7 months postoperatively, compared with chemotherapy alone (median survival 23 versus 13 months). In a recent comparable study, patients with nonmetastatic PNEC $\leq 2 \mathrm{~cm}$ who underwent resection, had a median survival of 29 months, while patients who were left to best supportive care had a median survival of 5 months (versus 2 months in our study) ${ }^{217}$. The three-year survival from time of metastasis after resection of nonmetastatic PNEC in our study was surprisingly high (45\%). This is consistent with a similar current report of a 5-year survival of $43 \%$ after surgical resection of nonmetastatic PNEC ${ }^{26}$. An important observation, and essential limitation, is that the case number of patients with nonmetastatic PNEC undergoing surgery in these studies, including our paper, was generally low ranging from 20 to 26 .

According to the current ENETS consensus guidelines for GEP-NECs, combination of postoperative platinum-based chemotherapy with local treatment consisting of surgery, radiotherapy, or both probably offers the greatest likelihood of long-term survival for patients with nonmetastatic disease, irrespective of the exact site of the primary ${ }^{106}$. As most of the patients in our study were treated with platinum-based chemotherapy regimens (81\%), our data support this recommendation for nonmetastatic PNEC and suggests surgery and adjuvant systemic platinum-based chemotherapy. 
The impact of surgery on the prognosis of patients with locally advanced PNEC, defined as ENETS T-stages T3 or T4, has not been evaluated yet. However, the results of Paper III, which includes 19 patients with a T3 or T4 PNEC, indicate that surgery combined with chemotherapy may improve survival in locally advanced disease when compared with chemotherapy alone. The current NANETS consensus guidelines provide only an expert opinion on this matter, which supports surgery, if the risk of morbidity is low and the risk of intestinal obstruction is high $^{118}$. The results of our study are in accordance with this recommendation. Furthermore, we would suggest surgery for resectable locally advanced nonmetastatic PNEC for selected patients, despite a higher risk for a marginpositive resection. This is supported by an observed improved survival after R0/R1 resections of PNEC, compared with $\mathrm{R} 2$ resections $^{218}$.

Most patients with PNEC develop distant metastases, which are often present already at diagnosis ${ }^{219}$. This reduces the prospects for long-term survival. Eightyfive percent $(n=101)$ of the patients in our study had synchronous metastatic disease. Surgery for metastatic disease in patients with PNEC is not recommended in the current ENETS and NANETS guidelines ${ }^{106,117,118}$. This is also supported by a recent international consensus conference on the treatment of neuroendocrine liver metastasis ${ }^{220}$. Of the patients with synchronous metastatic disease in Paper III, 14 underwent surgical treatment for the primary tumor alone $(n=2)$ or the primary tumor and metastatic liver disease $(n=12)$. In spite of the limitations of our study, the reported three-year survival rate of $69 \%$ among 12 patients with surgical treatment of all metastatic disease questions the very rigid current international guideline recommendations. Another important finding in our study is that surgical treatment of both the primary tumor and metastatic liver disease combined with systemic chemotherapy may improve the survival of patients with metastatic PNEC compared with chemotherapy alone (median survival 29 versus 13 months). This is coherent with a comparable study where overall survival after surgery of metastatic PNEC was 24 months with a 5-year survival of 21\%, among 13 patients who underwent surgery for metastatic liver disease ${ }^{221}$. 
One important limitation of the study reported in Paper III is that predefined criteria for surgical treatment were lacking. When considering the retrospective nature of the study and the fact that the patients included were from hospitals in different countries, this bias seemed difficult to eliminate. Variations in surgical treatment protocols for PNEC patients among the participating hospitals should be assumed. One observation suggesting this was that surgical activity on PNEC patients differed among the hospitals.

In Paper III, we observed that most nonmetastatic PNECs recurred or metastasized within one year after resection (in 13 out of 14 patients). This might suggest the presence of occult metastases at diagnosis. Thus, postoperative platinum-based chemotherapy should probably always be considered for patients with PNEC, regardless of the stage of the disease and provided the treatment is tolerated $^{106}$. The multivariate regression analysis in Paper III showed that $>4$ courses of postoperative chemotherapy was a significant factor of improved survival compared with $1-4$ courses ( $\leq 4$ versus $>4$ courses; HR 3.1, CI 1.9-5.2). This finding is influenced by a selection bias as some patients will die or show clinical deterioration before receiving more than four courses of chemotherapy. However, based on these findings and being aware of the limited clinical evidence available, we recommend upfront surgery of nonmetastatic disease with postoperative systemic chemotherapy of more than four courses. This is also supported by expert opinion reported in the current NANETS guidelines ${ }^{118}$.

In Paper III, we found the Ki67 index to be another significant predictor of survival in patients with PNEC $(\geq 55 \%$ versus $<55 \%$; HR 2.2, CI 1.3-3.6). As prognosis of GEP-NECs correlates with the Ki67 index, tumors with a very high Ki67 are more aggressive than tumors with a Ki67 just above $20 \%{ }^{113,222}$. At the same time, GEP-NECs with a high Ki67 ( $\geq 55 \%$ ) do respond better on systemic platinum-based chemotherapy than GEP-NECs with a lower Ki67 $(<55 \%)^{113}$. Based on these observations it could be argued that upfront surgery should be performed for PNECs with a Ki67 < 55\% and neoadjuvant systemic platinum- 
based chemotherapy for PNECs with a Ki67 $\geq 55 \%$. There are, so far, no systematic studies that have assessed the use of neoadjuvant chemotherapy in patients with apparently nonmetastatic PNEC. However, given the early manifestation of recurrent metastatic disease after surgery for apparently nonmetastatic PNEC in our study, measures to elucidate the effect of neoadjuvant chemotherapy on survival in these patients seem warranted. Based on what we currently know about GEP-NECs in general and PNECs in particular, we recommend neoadjuvant systemic platinum-based chemotherapy for PNECs with a $\mathrm{Ki} 67 \geq 55 \%$. However, this should be considered as nothing more than an expert opinion.

A discrepancy in grading defined by mitotic rate and $\mathrm{Ki} 67$ has been observed in up to $44 \%$ of $\mathrm{PNENs}^{223}$. PNENs with a mitotic rate within the $\mathrm{G} 2$ range and a Ki67 index corresponding to G3 have been described ${ }^{165,224,225}$. Such "grade-discordant" PNENs were found to have better prognosis compared with true PNECs (median survival 54 versus 11 months), but a worse outcome compared with "gradeconcordant" PNENs (median survival 54 versus 68 months) ${ }^{165}$. A further recent observation that exemplifies the heterogeneity of PNECs is the difference in response rate to first-line platinum-based systemic chemotherapy among patients with a GEP-NEC depending on whether they had a Ki67 above or below 55\%. Interestingly, the response rate correlated with the Ki67 index (response rate $42 \%$ with $\mathrm{Ki} 67$ above $55 \%$ versus $14 \%$ with $\mathrm{Ki} 67$ below $55 \%)^{113}$. Grade concordancy, as described in this section, was not included in our study. However, based on the results of other aforementioned studies, the existence of a "grade-discordant" group of PNEC with unique clinical features and the association between the Ki67 index and effect of platinum-based chemotherapy of PNEC, imply the need for modification of the current WHO 2010 grading system for PNENs ${ }^{31}$.

Coming back to the issue raised earlier about lymph node sampling in patients with PNENs, it is unknown if peripancreatic lymphadenectomy improves survival in patients with PNEC since this has not been evaluated in clinical trials. However, 
as lymph node stage predicts prognosis, peripancreatic lymphadenectomy should probably be performed routinely in patients undergoing surgery for PNEC.

Considering the aggressive nature of PNEC, one can assume that the performance status (PS) of patients suffering from PNEC deteriorates rapidly. This assumption is in line with our study, where $20 \%$ of the patients presented with a WHO PS equal to or above 2. We also found the grade of PS to be a significant variable of survival (PS 1 versus PS 0; HR 1.9, CI 1.0-3.3; PS $\geq 2$ versus PS 0; HR 7.5, CI 3.416.6).

As mentioned before, there are important limitations to our study other than the small sample size, especially for the patients who underwent surgical treatment, and the retrospective design, which both explain a selection bias of the study. The lack of a centralized histopathology reevaluation of the tissues from the enrolled patients has led to comparison of results from different pathologists over many years, which may diverge in terms of tissue staining techniques and Ki67 index assessment procedures. The lack of Ki67 values from both primary tumor and metastatic tissue was another limitation. For seven patients, all of whom underwent surgery, Ki67 was determined from both the primary and metastatic tissue. Metastatic tissue generally had a higher Ki67 than primary tumor tissue, consistent with other reports of other GEP-NENs ${ }^{226,227}$. However, in our study, the mean Ki67 value for those who did not undergo surgery was $48 \pm 26 \%$, whereas the mean Ki67 value of the tissues from the surgically treated patients was $47 \pm 26 \%$. Based on these data, the two groups seemed comparable in terms of tumor biology as defined by Ki67. Another limitation of the study was absence of data on the total hepatic tumor burden for patients with metastatic liver disease as well as heterogeneity of the chemotherapy regimens that were administered. However, given the very low incidence of PNECs and the current shortage of evidence concerning surgical treatment of this patient group, we believe that Paper III gives important novel knowledge about multimodal management of PNEC. It raises important questions that should be further investigated in future studies. 
In conclusion, the findings of our study suggest that resection of the primary tumor should be considered, and additionally, that patients with resectable PNEC and resectable synchronous metastatic disease should be considered for surgery of both the primary tumor and the metastases. This is supported by recent reports of the effect of surgery on survival for PNENs across all stages of disease, including PNECs $^{49,109}$. Since work began on this thesis, a novel interest in surgical treatment of PNEC has been noted $26,217,218$, although the underlying clinical evidence for a surgical approach is still scarce and prospective trials are lacking. There is a need for raised awareness of this evolving field in pancreatic surgery. Further efforts should be made to increase the attention of surgeons to surgery of PNEC as a part of a multimodal management of patients with PNEC aiming at improved survival. The therapeutic approach for nonmetastatic PNEC is at present neither consistent nor uniform ${ }^{228}$. Future studies on surgical treatment of PNEC should focus on the establishment of standardized sequences of treatment, especially the combined use of platinum-based chemotherapy pre- and postoperatively ${ }^{229}$. PNECs should be studied as a separate entity with precise reporting of their characteristics in future trials $^{230}$. Moreover, initiatives should be taken to plan and conduct prospective multicenter studies. We propose the need for a prospective trial on surgical treatment combined with platinum-based chemotherapy of resectable metastatic disease versus platinum-based chemotherapy alone. As the current guidelines are exclusively based on expert opinions, and considering the latest clinical data on this matter that diverge from the recommendations, we could expect that the expert opinions may be modified in the near future as more clinical data on surgery of PNEC become available. 


\section{Genomic imbalance profiling in PNENs}

As mentioned earlier, most PNENs are sporadic and nonfunctioning ${ }^{11,15,16}$. In the patient cohorts included in the clinical part of this thesis, around half of the patients underwent removal of a sporadic nonfunctioning PNEN (Papers I and II).

In Paper IV, we performed karyotyping and CGH on a small series of sporadic nonfunctioning PNENs. The aim was to describe genomic imbalances in the whole cohort and to stratify these by known factors of prognosis. In doing so, cytogenetic data were compared between tumor samples that had been stratified by three known prognostic factors: the Ki67 index, metastatic status, and size of the primary tumor $^{184}$. In Paper I, we showed that a Ki67 cut-off value of 5\% is a prognostic factor of survival after laparoscopic surgery for PNENs, something that has also been seen by others ${ }^{44}$. We therefore chose a Ki67 cut-off value of 5\%. Our study was the first to correlate data obtained by $\mathrm{CGH}$ with karyotyping and cell proliferation (Ki67 index) in patients with PNENs.

We found copy number changes to be common in sporadic nonfunctioning PNENs, with an ANCA index of 12. Common gains were scored at 5p12-13, 4q1324, 5p15, 5q11-31, and 9q21-22, whereas common losses were scored at 11p11, 11p14-15, 11q23, 11p12-13, and 11q22. After subdivision of the results according to Ki67, metastatic status, and tumor size, we found a higher ANCA index in samples from patients with high Ki67, metastatic disease, and a large-sized tumors ( $\geq 3.5 \mathrm{~cm}$ ), which implicates acquired genomic imbalances in sporadic nonfunctioning PNEN progression. The most frequent genomic imbalance in our series was loss of $11 \mathrm{p} 11$, which was not only seen in tumor samples of more "aggressive" behavior but occurred frequently also in samples with low Ki67, nonmetastatic disease, and small tumor size. We interpreted the loss of $11 \mathrm{p} 11$ as a potential early event in PNEN tumorigenesis and proposed the chromosomal band 11 p11 as a possible location of relevant hitherto unrecognized tumor suppressor genes in sporadic nonfunctioning PNENs. 
Previous studies of genomic imbalances in nonfunctioning PNENs detected by CGH have examined, to the best of our knowledge, 54 cases $^{143,144,146,231 .}$ Common gains were described in 7q, 17q, and 20q, whereas common losses were seen in 6q, 11p, and 11q. The MEN1 gene, located in chromosomal band 11q13.1, is associated with development of some PNENs ${ }^{129}$. The presence of copy number losses at 11q might indicate MEN1 mutations. In our study, loss of chromosome band 11q13 was not particularly common as it was seen in only four sporadic nonfunctioning PNENs (3 mild and 1 aggressive), which is consistent with the presence of mutations in the MEN1 gene in less than half of all patients with PNENs ${ }^{129}$. Other associations between the genomic imbalances found in our study and genes associated with PNEN tumorigenesis are suggested in Table 4.

Out of ten samples that were karyotyped, we found one abnormal karyotype with an extra chromosome 12 as the only clonal aberration, a change that was confirmed by CGH. Previous studies of genomic imbalances in PNENs have reported seven cases with karyotypic aberrations, but no clearly nonrandom, let alone specific, chromosomal abnormalities ${ }^{140-142}$.

In summary, Paper IV indicates the existence of distinct cytogenetic patterns in sporadic nonfunctioning PNENs, depending on their Ki67 index (cell proliferation), metastatic status, and size. In particular, loss of chromosomal band 11 p11 might indicate the site of a primary, or at least early, pathogenetic event in these neoplasms. Our study represents a step towards molecular pathological classification of sporadic nonfunctioning PNENs, despite important limitations such as the small sample size and the lack of validation of the findings in larger series. Future investigations should focus on potential primary events of tumorigenesis on chromosome band $11 \mathrm{p} 11$.

\section{Genomic expression profiling in PNENs}

In Paper V, we performed high-throughput sequencing and bioinformatics analysis of a series of sporadic nonfunctioning PNENs. The aim was to identify 
expression patterns that could be important for molecular differentiation of tumor aggressiveness in a small series of sporadic nonfunctioning PNENs. To our knowledge, the study is the first published report on high-throughput transcriptome sequencing of sporadic nonfunctioning PNENs.

Tumor aggressiveness was dichotomized into "mild" and "aggressive" disease, based on three parameters. These parameters were: the degree of cell proliferation as indicated by the $\mathrm{Ki} 67$ index $(<$ or $\geq 5 \%)$, the presence of metastatic disease at time of diagnosis, and the size of the primary tumor $(<$ or $\geq 3.5 \mathrm{~cm})$. "Aggressive tumors" were defined by a Ki67 index $\geq 5 \%$, metastatic disease at time of surgery, and tumor size $\geq 3.5 \mathrm{~cm}$.

We found a set of 309 protein-coding genes that were significantly differentially expressed according to tumor aggressiveness, of which 166 were upregulated and 143 downregulated in the aggressive disease group. Some of the most upregulated genes were involved in DNA packaging (HIST1H2AL, HIST1H2BF), ability to taste (TAS2R8), chromosome structuring (TRIP13), cytoskeleton structuring $(A D D 2)$, and cell-cell signaling (WNT3, ITPKA and GDF15). Among the most downregulated genes were genes involved in neuronal differentiation $(M Y T 1 L)$, cytoskeleton structuring (KRT27), cell-cell signaling $(G A B R P)$, and immune reactions (CTSE). Although some of these genes have already been associated with malignancies, others have never before been linked to cancer.

Four of the most upregulated genes were of particular interest: HIST1H2AL, $H I S T 1 H 2 B F, A D D 2$, and WNT3. HIST1H2AL and HIST1H2BF, also known as histone cluster $1 \mathrm{H} 2 \mathrm{al}$ and histone cluster $1 \mathrm{H} 2 \mathrm{bf}$, express two of the four core histones that are essential for packaging of DNA in nucleosomes. Histones play an important part in the process of gene expression ${ }^{232}$. Our data suggest, for the first time, a correlation between aggressiveness of sporadic nonfunctioning PNENs and expression of histones encoded by HIST1H2AL and HIST1H2BF. An interesting 
observation was the presence of copy number gain on $6 \mathrm{p} 22$ in one patients with aggressive disease in Paper IV. This band covers the gene locus of HIST1H2AL and HIST1H2BF (6p22.1) (Table 4). Correlation between grade of pulmonary neuroendocrine tumors and expression of histone $\mathrm{H} 1.5$ has been reported $^{232}$. Expression of HIST1H2AL and HIST1H2BF might similarly be of use as an immunohistochemical marker of tumor aggressiveness in sporadic nonfunctioning PNENs. This could be further investigated in future studies using commercially available antibodies. In our study, $A D D 2$ was significantly overexpressed in tumors associated with high aggressiveness. Overexpression of ADD2 (adducin 2) in NENs has not been reported previously. However, expression of adducin genes correlates with grade of proliferation and Ki67 expression in basal cell carcinoma and oral/cutaneous squamous cell carcinoma ${ }^{233}$, and may therefore represent another yet undescribed marker of tumor aggressiveness in sporadic nonfunctioning PNENs.

One of the main findings in Paper $\mathbf{V}$ was the upregulation of WNT3 (17q21) in sporadic nonfunctioning PNENs with aggressive behavior. This was in line with the findings of copy number gains on 17q21 in 3 of the 13 analyzed tumor samples, all of which showed aggressive behavior. These findings suggest a relationship between the level of WNT3 expression and the grade of tumor aggressiveness in sporadic nonfunctioning PNENs. WNT3 encodes a secreted signaling protein associated with regulation of cell fate and patterning during embryogenesis through activation of the Wnt signaling pathway, as illustrated in Figure 14. Cell proliferation is stimulated through Wnt signaling, which increases nuclear and cytoplasmatic levels of beta-catenin ${ }^{234}$. Beta-catenin leads to increased expression of proteins such as cyclin D1 and MYC that both control the G1 to S phase transition in the cell cycle ${ }^{235}$. Previous studies have shown a relationship between the expression of WNT3 and development of colorectal cancer ${ }^{236}$, malignant mesothelioma ${ }^{237}$, non-small cell lung cancer $^{238}$, breast cancer $^{239}$, and cholangiocarcinoma ${ }^{240}$. Moreover, evidence exists that WNT3 expression stimulates pancreatic islet beta-cell proliferation ${ }^{241}$. Aberrant Wnt signaling has 
also been correlated with decreased expression of Wnt inhibitors in a pancreatic neuroendocrine cell line $\mathrm{e}^{242,243}$. There is also evidence suggesting that menin (encoded by MEN1) activates the Wnt signaling pathway resulting in inhibition of islet tumor cell proliferation in a mouse model ${ }^{244}$. Further, it was recently been demonstrated that neurotensin is a target of the Wnt signaling pathway and promotes growth of pancreatic neuroendocrine cells ${ }^{245}$. These observations strengthen the idea of the Wnt signaling pathway as a potential target for molecular markers of prognosis in patients with sporadic nonfunctioning PNEN.

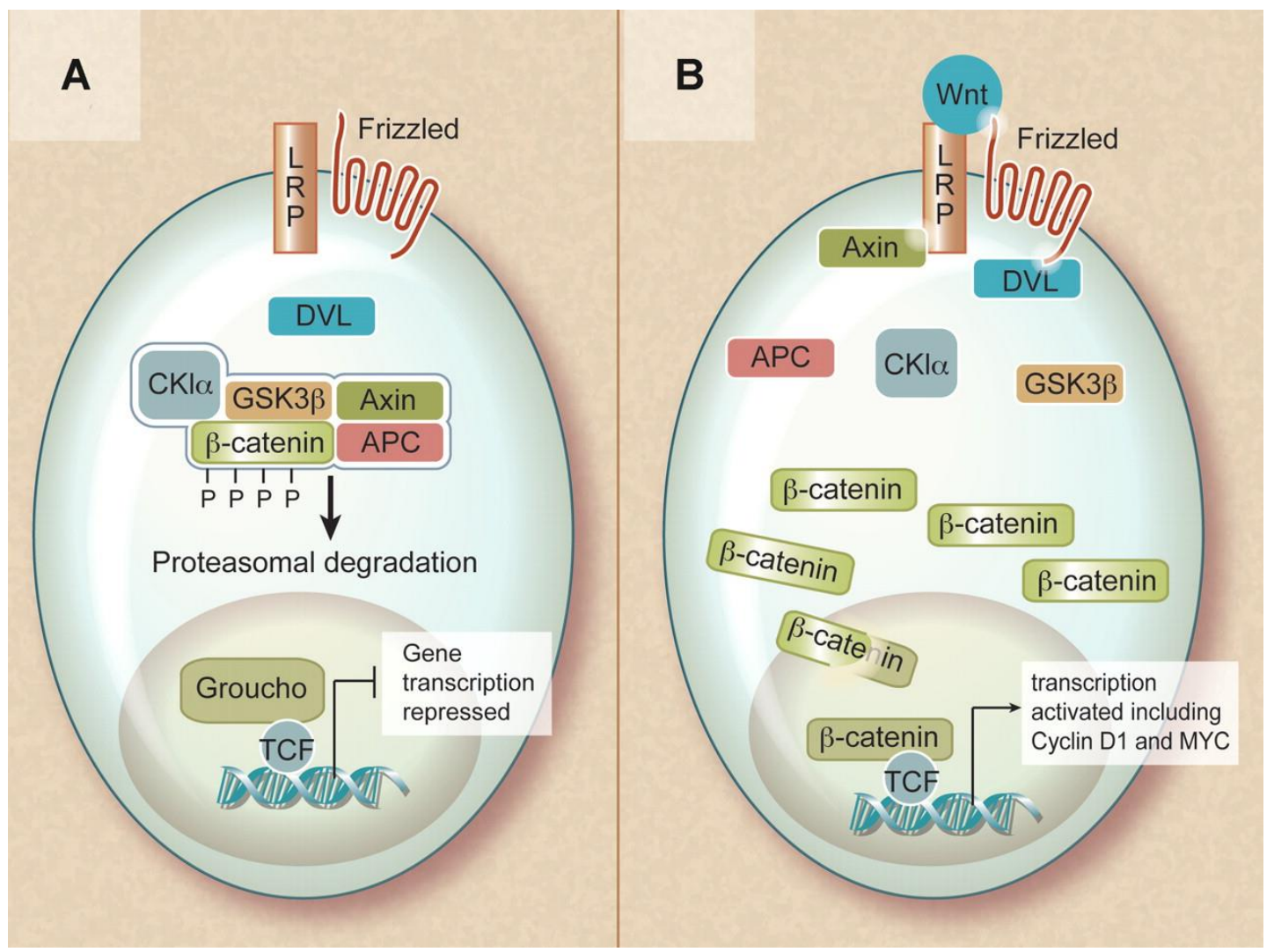

Figure 14. The Wnt signaling pathway (from Ansell $\left.{ }^{246}\right)$. A, in the absence of a Wnt signal. $\boldsymbol{B}$, in the presence of a Wnt signal

The association between PI3K/Akt/mTOR pathway genes, novel genes associated with PNENs, genomic imbalance profiling results of Paper IV, and genomic expression profiling results of Paper $\mathbf{V}$ is summarized in Table 4. 
Table 4. Association between PI3K/Akt/mTOR pathway genes, novel genes associated with PNENs, from the genomic imbalance profiling results of Paper IV, and the genomic expression profiling results of Paper $\boldsymbol{V}$. "Aggressive" is defined as metastatic sporadic nonfunctioning PNEN with Ki67 $\geq 5 \%$. "Mild" is defined as nonmetastatic sporadic nonfunctioning PNEN with Ki67<5\%. *Upregulated gene in aggressive versus mild disease

\begin{tabular}{|c|c|c|c|c|}
\hline Gene & Cytogenetic band & $\begin{array}{l}\text { Copy number gain } \\
\text { (Paper IV) }\end{array}$ & $\begin{array}{c}\text { Copy number loss } \\
\text { (Paper IV) }\end{array}$ & $\begin{array}{c}\text { Statistically } \\
\text { significant } \\
\text { differential } \\
\text { expression (yes/no) } \\
(\text { Paper V)* }\end{array}$ \\
\hline \multicolumn{5}{|c|}{ PI3K/Akt/mTOR pathway genes (PNENs in general) } \\
\hline MEN1 & $11 q 13.1$ & No & $\begin{array}{c}11 \mathrm{q} 13 \text { in } 3 \text { mild and } \\
1 \text { aggressive }\end{array}$ & No \\
\hline$D A X X$ & $6 \mathrm{p} 21.32$ & $\begin{array}{l}6 \mathrm{p} 21 \text { in } 1 \\
\text { aggressive }\end{array}$ & $\begin{array}{c}6 \mathrm{p} 21 \text { in } 1 \\
\text { aggressive }\end{array}$ & No \\
\hline $\operatorname{ATRX}$ & $\mathrm{Xq} 21.1$ & No & No & No \\
\hline PTEN & $10 q 23.31$ & $\begin{array}{c}10 \mathrm{q} 23 \text { in } 1 \text { mild and } \\
1 \text { aggressive }\end{array}$ & No & No \\
\hline TSC2 & $16 \mathrm{p} 13.3$ & $\begin{array}{l}16 \mathrm{p} 13 \text { in } 1 \\
\text { aggressive }\end{array}$ & $\begin{array}{c}16 \mathrm{p} 13 \text { in } 2 \\
\text { aggressive and } 1 \\
\text { mild }\end{array}$ & No \\
\hline$P I K 3 C A$ & $3 q 26.32$ & No & $3 q 26$ in 1 mild & No \\
\hline TP53 & $17 \mathrm{p} 13.1$ & $\begin{array}{l}17 \mathrm{p} 13 \text { in } 2 \\
\text { aggressive }\end{array}$ & No & No \\
\hline \multicolumn{5}{|c|}{ Novel genes (sporadic nonfunctioning PNENs) } \\
\hline$H I S T 1 H 2 A L$ & $6 \mathrm{p} 22.1$ & $\begin{array}{c}6 \mathrm{p} 22 \text { in } 1 \\
\text { aggressive }\end{array}$ & No & Yes, upregulated \\
\hline HISTIH $2 B F$ & $6 \mathrm{p} 22.1$ & $\begin{array}{c}6 \mathrm{p} 22 \text { in } 1 \\
\text { aggressive }\end{array}$ & No & Yes, upregulated \\
\hline$A D D 2$ & $2 \mathrm{p} 13.3$ & $\begin{array}{l}2 \mathrm{p} 13 \text { in } 1 \\
\text { aggressive }\end{array}$ & $\begin{array}{l}2 \mathrm{p} 13 \text { in } 1 \\
\text { aggressive }\end{array}$ & Yes, upregulated \\
\hline WNT3 & $17 \mathrm{q} 21$ & $\begin{array}{l}17 \mathrm{q} 21 \text { in } 3 \\
\text { aggressive }\end{array}$ & No & Yes, upregulated \\
\hline \multicolumn{5}{|c|}{ WNT signaling target genes (sporadic nonfunctioning PNENs) } \\
\hline BIRC5 (survivin) & $17 q 25.3$ & $\begin{array}{l}17 q 25 \text { in } 3 \\
\text { aggressive }\end{array}$ & No & Yes, upregulated \\
\hline
\end{tabular}

After publication of Paper V, we have examined different Wnt target genes among the 309 protein-coding genes that were found to be significantly differentially expressed. One interesting finding was the significant upregulation of the BIRC5 gene $(17 \mathrm{q} 25.3)$ in aggressive disease (logFC -2.1). BIRC5 encodes survivin, which is an apoptosis inhibitor normally present in fetal tissue but absent from terminally differentiated tissue. It has previously been reported to be upregulated in PNENs 
and has been associated with poor survival ${ }^{247,248}$. An interesting link between the results of Papers IV and $\mathbf{V}$ is the copy number loss of $17 q 21$ and $17 q 25$ in three samples categorized as "aggressive disease" (Paper IV) and the identification of significantly upregulated genes in sporadic nonfunctioning PNENs with aggressive behavior on the same chromosomal bands (WNT3 on 17q21 and BIRC5 on 17q25.3) (Paper V). As mentioned above, previous studies have also shown gains of $17 \mathrm{q}$ in nonfunctioning PNENs.

In summary, Paper $\mathbf{V}$ reveals novel patterns of correlation between tumor aggressiveness and genomic expression in sporadic nonfunctioning PNENs. We identified upregulation of genes involved in DNA packaging, cytoskeleton structuring, and cell-cell-signaling in aggressive disease, which could indicate yet undescribed molecular targets for biomarkers and drugs in histones and/or the Wnt signaling pathway. Our study has important limitations and the results should be interpreted with care. One limitation is the small sample size, only 11 tumors, which makes the results difficult to generalize. Further, we did not validate our findings by means of real-time PCR and/or immunohistochemistry in the same set of tumor samples. Another way of validating the results could have been to perform real-time PCR on a large independent cohort of sporadic nonfunctioning PNENs. However, despite the clear limitations of the study, its preliminary results give important and new knowledge about potentially important biomarkers for sporadic nonfunctioning PNENs. In particular, more investigation is needed to clarify the role of the Wnt signaling pathway in the tumorigenesis of sporadic nonfunctioning PNENs. 


\section{Conclusions}

\section{Paper I}

- Laparoscopic surgery for PNENs is feasible with acceptable overall surgical morbidity and a good long-term prognosis

- In laparoscopic surgery for PNENs, a Ki67 index cut-off value of 5\% is a significant prognostic factor

\section{Paper II}

- In patients with locally advanced PNET, pancreatic surgery with vascular reconstruction is feasible with acceptable surgical morbidity and short-term prognosis

\section{Paper III}

- In patients with PNEC, combined surgical treatment and chemotherapy improves survival compared with chemotherapy alone

- In patients with PNEC, resection of the primary tumor is an independent factor of improved survival

\section{Paper IV}

- In sporadic nonfunctioning PNENs, the number of genomic imbalances seems to correlate directly with cell proliferation, tumor size and metastatic status. Loss of genomic material from chromosomal band 11p11 might represent a primary pathogenetic event

\section{Paper V}

- In sporadic nonfunctioning PNENs, a higher level of tumor aggressiveness seems to be associated with upregulation of genes involved in regulation of the cell cycle and cell division, such as genes of the Wnt signaling pathway

Based on the clinical studies of this thesis and the discussed evidence on surgical management of patients with PNENs, we finally propose two algorithms (in the Appendix) that should help surgeons choose the optimal surgical treatment. 


\section{References}

(1) Nicholls AG. Simple Adenoma of the Pancreas arising from an Island of langerhans. $J$ Med Res 1902;8(2):385-395.

(2) Modlin IM, Shapiro MD, Kidd M. Siegfried Oberndorfer: origins and perspectives of carcinoid tumors. Hum Pathol 2004;35(12):1440-1451.

(3) Oberndorfer S. Karzinoide Tumoren des Duenndarms. Frankfurter Zeitschrift für Pathologie 1907;1(34).

(4) Wilder RM, Allan FN, Power MH, Robertson HE. Carcinoma of the islands of the pancreas - hyperinsulism and hypoglycemia. JAMA 1927;89(5):348-355.

(5) Howland G, Campbell WR, Maltby EJ, Robinson WL. Dysinsulinism - convulsions and coma due to islet cell tumor of the pancreas, with operation and cure. JAMA 1929;93(9):674-679.

(6) Feyrter F. Ueber diffuse endokrine epitheliale Organe. Zentralbl Innere Med 1938;(545):31-41.

(7) Williams ED, Sandler M. The classification of carcinoid tum ours. Lancet 1963;1(7275):238-239.

(8) Pearse AG. 5-hydroxytryptophan uptake by dog thyroid 'C' cells, and its possible significance in polypeptide hormone production. Nature 1966;211(5049):598-600.

(9) Pearse AG. The diffuse endocrine system and the implications of the APUD concept. Int Surg 1979;64(2):5-7.

(10) Rindi G, Leiter AB, Kopin AS, Bordi C, Solcia E. The "normal" endocrine cell of the gut: changing concepts and new evidences. Ann N Y Acad Sci 2004;1014:1-12.

(11) Halfdanarson TR, Rabe KG, Rubin J, Petersen GM. Pancreatic neuroendocrine tumors (PNETs): incidence, prognosis and recent trend toward improved survival. Ann Oncol 2008;19(10):1727-1733.

(12) Milan SA, Yeo CJ. Neuroendocrine tumors of the pancreas. Curr Opin Oncol 2012;24(1):46-55.

(13) Rindi G, Bordi C, La RS, Solcia E, Delle FG. Gastroenteropancreatic (neuro)endocrine neoplasms: the histology report. Dig Liver Dis 2011;43 Suppl 4:S356-S360.

(14) Kulke MH, Bendell J, Kvols L, Picus J, Pommier R, Yao J. Evolving diagnostic and treatment strategies for pancreatic neuroendocrine tumors. J Hematol Oncol 2011;4:29.

(15) Falconi M, Eriksson B, Kaltsas $G$ et al. Consensus Guidelines Update for the Management of Functional p-NETs (F-p-NETs) and Non-Functional p-NETs (NF-pNETs). Neuroendocrinology 2016.

(16) Bilimoria KY, Tomlinson JS, Merkow RP et al. Clinicopathologic features and treatment trends of pancreatic neuroendocrine tumors: analysis of 9,821 patients. $J$ Gastrointest Surg 2007;11(11):1460-1467.

(17) Evans DB, Skibber JM, Lee JE et al. Nonfunctioning islet cell carcinoma of the pancreas. Surgery 1993;114(6):1175-1181.

(18) Liang H, Wang P, Wang XN, Wang JC, Hao XS. Management of nonfunctioning islet cell tumors. World J Gastroenterol 2004;10(12):1806-1809.

(19) Bartsch DK, Schilling T, Ramaswamy A et al. Management of nonfunctioning islet cell carcinomas. World J Surg 2000;24(11):1418-1424.

(20) Oberg K. Pancreatic endocrine tumors. Semin Oncol 2010;37(6):594-618.

(21) Boyar CR, Aagnes B, Thiis-Evensen E, Tretli S, Bergestuen DS, Hansen S. Trends in Incidence of Neuroendocrine Neoplasms in Norway: A Report of 16,075 Cases from 1993 through 2010. Neuroendocrinology 2015.

(22) Fitzgerald TL, Hickner ZJ, Schmitz M, Kort EJ. Changing incidence of pancreatic neoplasms: a 16-year review of statewide tumor registry. Pancreas 2008;37(2):134138.

(23) Sandvik OM, Soreide K, Gudlaugsson E, Kvaloy JT, Soreide JA. Epidemiology and classification of gastroenteropancreatic neuroendocrine neoplasms using current coding criteria. Br J Surg 2016;103(3):226-232. 
(24) Yao JC, Hassan M, Phan A et al. One hundred years after "carcinoid": epidemiology of and prognostic factors for neuroendocrine tumors in 35,825 cases in the United States. J Clin Oncol 2008;26(18):3063-3072.

(25) Bilimoria KY, Talamonti MS, Tomlinson JS et al. Prognostic score predicting survival after resection of pancreatic neuroendocrine tumors: analysis of 3851 patients. Ann Surg 2008;247(3):490-500.

(26) Fischer L, Bergmann F, Schimmack S et al. Outcome of surgery for pancreatic neuroendocrine neoplasms. Br J Surg 2014;101(11):1405-1412.

(27) Kuo EJ, Salem RR. Population-level analysis of pancreatic neuroendocrine tumors 2 cm or less in size. Ann Surg Oncol 2013;20(9):2815-2821.

(28) Kimura W, Kuroda A, Morioka Y. Clinical pathology of endocrine tumors of the pancreas. Analysis of autopsy cases. Dig Dis Sci 1991;36(7):933-942.

(29) Ehehalt F, Saeger HD, Schmidt CM, Grutzmann R. Neuroendocrine tumors of the pancreas. Oncologist 2009;14(5):456-467.

(30) Campbell F, Verbeke CS. Pathology of the pancreas: a practical approach. London: Springer Verlag; 2013.

(31) Bosman FT, Carneiro F, Hruban RH. WHO Classification of tumours of the digestive system. Lyon: International Agency for Research on Cancer (IARC); 2010.

(32) Gerdes J, Schwab U, Lemke H, Stein H. Production of a mouse monoclonal antibody reactive with a human nuclear antigen associated with cell proliferation. Int J Cancer 1983;31(1):13-20.

(33) Zhu LM, Tang L, Qiao XW et al. Differences and Similarities in the Clinicopathological Features of Pancreatic Neuroendocrine Tumors in China and the United States: A Multicenter Study. Medicine (Baltimore) 2016;95(7):e2836.

(34) Rindi G, Kloppel G, Alhman H et al. TNM staging of foregut (neuro)endocrine tumors: a consensus proposal including a grading system. Virchows Arch 2006;449(4):395-401.

(35) Sobin LH, Gospodarowicz MK, Wittekind C. UICC: TNM classification of malignant tumours. 7th. edn. ed. Oxford: Wiley-Blackwell; 2009.

(36) Ellison TA, Wolfgang CL, Shi C et al. A single institution's 26-year experience with nonfunctional pancreatic neuroendocrine tumors: a validation of current staging systems and a new prognostic nomogram. Ann Surg 2014;259(2):204-212.

(37) Strosberg JR, Cheema A, Weber JM et al. Relapse-free survival in patients with nonmetastatic, surgically resected pancreatic neuroendocrine tumors: an analysis of the AJCC and ENETS staging classifications. Ann Surg 2012;256(2):321-325.

(38) Rindi G, Falconi M, Klersy C et al. TNM staging of neoplasms of the endocrine pancreas: results from a large international cohort study. J Natl Cancer Inst 2012;104(10):764-777.

(39) Qadan M, Ma Y, Visser BC et al. Reassessment of the current American Joint Committee on Cancer staging system for pancreatic neuroendocrine tumors. J Am Coll Surg 2014;218(2):188-195.

(40) Yamaguchi T, Fujimori T, Tomita $\mathrm{S}$ et al. Clinical validation of the gastrointestinal NET grading system: Ki67 index criteria of the WHO 2010 classification is appropriate to predict metastasis or recurrence. Diagn Pathol 2013;8:65.

(41) Jernman J, Valimaki MJ, Louhimo J, Haglund C, Arola J. The novel WHO 2010 classification for gastrointestinal neuroendocrine tumours correlates well with the metastatic potential of rectal neuroendocrine tumours. Neuroendocrinology 2012;95(4):317-324.

(42) Goh BK, Chow PK, Tan YM et al. Validation of five contemporary prognostication systems for primary pancreatic endocrine neoplasms: results from a single institution experience with 61 surgically treated cases. ANZ J Surg 2011;81(1-2):79-85.

(43) Scarpa A, Mantovani W, Capelli P et al. Pancreatic endocrine tumors: improved TNM staging and histopathological grading permit a clinically efficient prognostic stratification of patients. Mod Pathol 2010;23(6):824-833. 
(44) Boninsegna L, Panzuto F, Partelli S et al. Malignant pancreatic neuroendocrine tumour: Lymph node ratio and Ki67 are predictors of recurrence after curative resections. Eur J Cancer 2011.

(45) Basturk O, Yang Z, Tang LH. Increased (> 20\%) Ki67 proliferation index in morphologically well differentiated pancreatic neuroendocrine tumors (PanNETs) correlates with decreased overall survival. Mod Pathol 2013;26(2S):423A.

(46) Cherif R, Gaujoux S, Couvelard A et al. Parenchyma-sparing resections for pancreatic neuroendocrine tumors. J Gastrointest Surg 2012;16(11):2045-2055.

(47) Chen H, Hardacre JM, Uzar A, Cameron JL, Choti MA. Isolated liver metastases from neuroendocrine tumors: does resection prolong survival? $J$ Am Coll Surg 1998;187(1):88-92.

(48) Solorzano CC, Lee JE, Pisters PWT et al. Nonfunctioning islet cell carcinoma of the pancreas: Survival results in a contemporary series of 163 patients. Surgery 2001;130(6):1078-1085.

(49) Keutgen XM, Nilubol N, Glanville J et al. Resection of primary tumor site is associated with prolonged survival in metastatic nonfunctioning pancreatic neuroendocrine tumors. Surgery 2015.

(50) Musunuru S, Chen H, Rajpal S et al. Metastatic neuroendocrine hepatic tumors: resection improves survival. Arch Surg 2006;141(10):1000-1004.

(51) Matthews BD, Smith TI, Kercher KW, Holder WD, Jr., Heniford BT. Surgical experience with functioning pancreatic neuroendocrine tumors. Am Surg 2002;68(8):660-665.

(52) Keutgen XM, Nilubol N, Kebebew E. Malignant-functioning neuroendocrine tumors of the pancreas: A survival analysis. Surgery 2016;159(5):1382-1389.

(53) Serrano PE, Cleary SP, Dhani N et al. Improved Long-Term Outcomes After Resection of Pancreatic Adenocarcinoma: A Comparison Between Two Time Periods. Ann Surg Oncol 2014.

(54) Jang JY, Kang MJ, Heo JS et al. A prospective randomized controlled study comparing outcomes of standard resection and extended resection, including dissection of the nerve plexus and various lymph nodes, in patients with pancreatic head cancer. Ann Surg 2014;259(4):656-664.

(55) Hill JS, McPhee JT, McDade TP et al. Pancreatic neuroendocrine tumors: the impact of surgical resection on survival. Cancer 2009;115(4):741-751.

(56) Gomez-Rivera F, Stewart AE, Arnoletti JP, Vickers S, Bland KI, Heslin MJ. Surgical treatment of pancreatic endocrine neoplasms. Am J Surg 2007;193(4):460-465.

(57) Legaspi A, Brennan MF. Management of islet cell carcinoma. Surgery 1988;104(6):1018-1023.

(58) Kazanjian KK, Reber HA, Hines OJ. Resection of pancreatic neuroendocrine tumors: results of 70 cases. Arch Surg 2006;141(8):765-769.

(59) Zerbi A, Capitanio V, Boninsegna L et al. Surgical treatment of pancreatic endocrine tumours in Italy: results of a prospective multicentre study of 262 cases. Langenbecks Arch Surg 2011;396(3):313-321.

(60) Boyar CR, Vatn M, Aabakken L, Bergestuen DS, Thiis-Evensen E. Survival and prognostic factors in well-differentiated pancreatic neuroendocrine tumors. Scand $J$ Gastroenterol 2014;49(6):734-741.

(61) Phan GQ, Yeo CJ, Cameron JL, Maher MM, Hruban RH, Udelsman R. Pancreaticoduodenectomy for selected periampullary neuroendocrine tumors: fifty patients. Surgery 1997;122(6):989-996.

(62) Park BJ, Alexander HR, Libutti SK et al. Operative management of islet-cell tumors arising in the head of the pancreas. Surgery 1998;124(6):1056-1061.

(63) Bassi C. Middle segment pancreatectomy: a useful tool in the management of pancreatic neoplasms. J Gastrointest Surg 2007;11(4):421-424.

(64) Muller MW, Friess H, Kleeff J et al. Middle segmental pancreatic resection: An option to treat benign pancreatic body lesions. Ann Surg 2006;244(6):909-918. 
(65) Gratian L, Pura J, Dinan M, Roman S, Reed S, Sosa JA. Impact of extent of surgery on survival in patients with small nonfunctional pancreatic neuroendocrine tumors in the United States. Ann Surg Oncol 2014;21(11):3515-3521.

(66) Muller MW, Friess H, Kleeff J et al. Is there still a role for total pancreatectomy? Ann Surg 2007;246(6):966-974.

(67) Gagner M, Pomp A, Herrera MF. Early experience with laparoscopic resections of islet cell tumors. Surgery 1996;120(6):1051-1054.

(68) Gagner M, Pomp A. Laparoscopic pancreatic resection: Is it worthwhile? J Gastrointest Surg 1997;1(1):20-25.

(69) Fernandez-Cruz L, Herrera M, Saenz A, Pantoja JP, Astudillo E, Sierra M. Laparoscopic pancreatic surgery in patients with neuroendocrine tumours: indications and limits. Best Pract Res Clin Endocrinol Metab 2001;15(2):161-175.

(70) Edwin B, Mala T, Mathisen O et al. Laparoscopic resection of the pancreas: a feasibility study of the short-term outcome. Surg Endosc 2004;18(3):407-411.

(71) Assalia A, Gagner M. Laparoscopic pancreatic surgery for islet cell tumors of the pancreas. World J Surg 2004;28(12):1239-1247.

(72) Mabrut JY, Fernandez-Cruz L, Azagra JS et al. Laparoscopic pancreatic resection: results of a multicenter European study of 127 patients. Surgery 2005;137(6):597-605.

(73) Kooby DA, Gillespie T, Bentrem D et al. Left-sided pancreatectomy: a multicenter comparison of laparoscopic and open approaches. Ann Surg 2008;248(3):438-446.

(74) Baker MS, Bentrem DJ, Ujiki MB, Stocker S, Talamonti MS. A prospective single institution comparison of peri-operative outcomes for laparoscopic and open distal pancreatectomy. Surgery 2009;146(4):635-643.

(75) Rosok BI, Marangos IP, Kazaryan AM et al. Single-centre experience of laparoscopic pancreatic surgery. Br J Surg 2010;97(6):902-909.

(76) Kooby DA, Hawkins WG, Schmidt CM et al. A multicenter analysis of distal pancreatectomy for adenocarcinoma: is laparoscopic resection appropriate? J Am Coll Surg 2010;210(5):779-7.

(77) Dedieu A, Rault A, Collet D, Masson B, Sa CA. Laparoscopic enucleation of pancreatic neoplasm. Surg Endosc 2011;25(2):572-576.

(78) Fernandez-Cruz L, Molina V, Vallejos R, Jimenez CE, Lopez-Boado MA, Ferrer J. Outcome after laparoscopic enucleation for non-functional neuroendocrine pancreatic tumours. HPB (Oxford) 2012;14(3):171-176.

(79) Abu HM, Hamdan M, Di FF, Pearce NW, Johnson CD. Laparoscopic versus open distal pancreatectomy: a clinical and cost-effectiveness study. Surg Endosc 2012;26(6):16701674.

(80) Limongelli P, Belli A, Russo G et al. Laparoscopic and open surgical treatment of leftsided pancreatic lesions: clinical outcomes and cost-effectiveness analysis. Surg Endosc 2012;26(7):1830-1836.

(81) Kim SC, Park KT, Hwang JW et al. Comparative analysis of clinical outcomes for laparoscopic distal pancreatic resection and open distal pancreatic resection at a single institution. Surg Endosc 2008;22(10):2261-2268.

(82) DiNorcia J, Lee MK, Reavey PL et al. One hundred thirty resections for pancreatic neuroendocrine tumor: evaluating the impact of minimally invasive and parenchymasparing techniques. J Gastrointest Surg 2010;14(10):1536-1546.

(83) Fernandez-Cruz L, Blanco L, Cosa R, Rendon H. Is laparoscopic resection adequate in patients with neuroendocrine pancreatic tumors? World J Surg 2008;32(5):904-917.

(84) Norton JA, Kivlen M, Li M, Schneider D, Chuter T, Jensen RT. Morbidity and mortality of aggressive resection in patients with advanced neuroendocrine tumors. Arch Surg 2003;138(8):859-866.

(85) Bertani E, Fazio N, Botteri E et al. Resection of the primary pancreatic neuroendocrine tumor in patients with unresectable liver metastases: possible indications for a multimodal approach. Surgery 2014;155(4):607-614.

(86) Hellman P, Andersson M, Rastad J et al. Surgical strategy for large or malignant endocrine pancreatic tumors. World J Surg 2000;24(11):1353-1360. 
(87) Bedirli A, Patiroglu TE, Sakrak O, Aritas Y. Portal vein resection for a portal vein thrombus caused by nonfunctioning islet cell carcinoma: report of a case. Surg Today 2004;34(9):802-804.

(88) Kawakami H, Kuwatani M, Hirano S et al. Pancreatic endocrine tumors with intraductal growth into the main pancreatic duct and tumor thrombus within the portal vein: a case report and review of the literature. Intern Med 2007;46(6):273-277.

(89) Yamato H, Kawakami H, Kuwatani M et al. Pancreatic carcinoma associated with portal vein tumor thrombus: three case reports. Intern Med 2009;48(3):143-150.

(90) Gundara JS, Alvarado-Bachmann R, Williams N et al. Multivisceral resection of pancreatic neuroendocrine tumours: a report of two cases. World J Surg Oncol 2011;9:93.

(91) Norton JA, Harris EJ, Chen Y et al. Pancreatic endocrine tumors with major vascular abutment, involvement, or encasement and indication for resection. Arch Surg 2011;146(6):724-732.

(92) Kleine M, Schrem H, Vondran FW, Krech T, Klempnauer J, Bektas H. Extended surgery for advanced pancreatic endocrine tumours. Br J Surg 2012;99(1):88-94.

(93) Sakamoto E, Hasegawa H, Ogiso S et al. Curative resection for a pancreatic endocrine carcinoma involving the portal vein. Hepatogastroenterology 2004;51(60):1849-1851.

(94) Akatsu T, Aiura K, Shimazu M et al. Successful pancreatectomy with en-bloc resection of the celiac artery and portal vein for pancreatic endocrine carcinoma. Hepatogastroenterology 2007;54(76):1269-1271.

(95) Ochiai T, Masuda T, Nishizawa $\mathrm{M}$ et al. Curative resection of a huge malignant pancreatic endocrine tumor by pancreatoduodenectomy with portal and superior mesenteric vein resection and reconstruction using the right ovarian vein: report of a case. Surg Today 2011;41(9):1260-1265.

(96) Tsuchikawa T, Kondo S, Hirano $S$ et al. Distal pancreatectomy and portal vein resection without vascular reconstruction for endocrine tumors with massive intraportal growth: report of a case. Hepatogastroenterology 2011;58(107-108):1029-1031.

(97) Harrison LE, Klimstra DS, Brennan MF. Isolated portal vein involvement in pancreatic adenocarcinoma. A contraindication for resection? Ann Surg 1996;224(3):342-347.

(98) van Geenen RC, ten Kate FJ, de Wit LT, van Gulik TM, Obertop H, Gouma DJ. Segmental resection and wedge excision of the portal or superior mesenteric vein during pancreatoduodenectomy. Surgery 2001;129(2):158-163.

(99) Fuhrman GM, Leach SD, Staley CA et al. Rationale for en bloc vein resection in the treatment of pancreatic adenocarcinoma adherent to the superior mesenteric-portal vein confluence. Pancreatic Tumor Study Group. Ann Surg 1996;223(2):154-162.

(100) Roder JD, Stein HJ, Siewert JR. Carcinoma of the periampullary region: who benefits from portal vein resection? Am J Surg 1996;171(1):170-174.

(101) Siriwardana HP, Siriwardena AK. Systematic review of outcome of synchronous portal-superior mesenteric vein resection during pancreatectomy for cancer. Br J Surg 2006;93(6):662-673.

(102) Chamberlain RS, Canes D, Brown KT et al. Hepatic neuroendocrine metastases: does intervention alter outcomes? J Am Coll Surg 2000;190(4):432-445.

(103) Grazi GL, Cescon M, Pierangeli F et al. Highly aggressive policy of hepatic resections for neuroendocrine liver metastases. Hepatogastroenterology 2000;47(32):481-486.

(104) Modlin IM, Oberg K, Chung DC et al. Gastroenteropancreatic neuroendocrine tumours. Lancet Oncol 2008;9(1):61-72.

(105) Metz DC, Choi J, Strosberg J et al. A rationale for multidisciplinary care in treating neuroendocrine tumours. Curr Opin Endocrinol Diabetes Obes 2012;19(4):306-313.

(106) Garcia-Carbonero R, Sorbye H, Baudin E et al. Consensus Guidelines for High Grade Gastro-Entero-Pancreatic (GEP) Neuroendocrine Tumours and Neuroendocrine Carcinomas (NEC). Neuroendocrinology 2016.

(107) Chabot J. Pancreatic neuroendocrine tumors: Primum non nocere. Surgery 2015.

(108) Kulke MH. Are neuroendocrine tumors going mainstream? J Clin Oncol 2013;31(4):404-405. 
(109) Huttner FJ, Schneider L, Tarantino I et al. Palliative resection of the primary tumor in 442 metastasized neuroendocrine tumors of the pancreas: a population-based, propensity score-matched survival analysis. Langenbecks Arch Surg 2015;400(6):715723.

(110) Schurr PG, Strate T, Rese K et al. Aggressive surgery improves long-term survival in neuroendocrine pancreatic tumors: an institutional experience. Ann Surg 2007;245(2):273-281.

(111) Fendrich V, Langer P, Celik I et al. An aggressive surgical approach leads to long-term survival in patients with pancreatic endocrine tumors. Ann Surg 2006;244(6):845-851.

(112) Basturk O, Tang L, Hruban RH et al. Poorly differentiated neuroendocrine carcinomas of the pancreas: a clinicopathologic analysis of 44 cases. Am J Surg Pathol 2014;38(4):437-447.

(113) Sorbye H, Welin S, Langer SW et al. Predictive and prognostic factors for treatment and survival in 305 patients with advanced gastrointestinal neuroendocrine carcinoma (WHO G3): the NORDIC NEC study. Ann Oncol 2013;24(1):152-160.

(114) Brenner B, Tang LH, Shia J, Klimstra DS, Kelsen DP. Small cell carcinomas of the gastrointestinal tract: clinicopathological features and treatment approach. Semin Oncol 2007;34(1):43-50.

(115) Casas F, Ferrer F, Farrus B, Casals J, Biete A. Primary small cell carcinoma of the esophagus: a review of the literature with emphasis on therapy and prognosis. Cancer 1997;80(8):1366-1372.

(116) Sorbye H, Westre B, Horn A. Curative surgery after neoadjuvant chemotherapy in metastatic poorly differentiated neuroendocrine carcinoma. Eur J Surg Oncol 2007;33(10):1209-1210.

(117) Strosberg JR, Coppola D, Klimstra DS et al. The NANETS consensus guidelines for the diagnosis and management of poorly differentiated (high-grade) extrapulmonary neuroendocrine carcinomas. Pancreas 2010;39(6):799-800.

(118) Kunz PL, Reidy-Lagunes D, Anthony LB et al. Consensus guidelines for the management and treatment of neuroendocrine tumors. Pancreas 2013;42(4):557-577.

(119) Oberg K, Knigge U, Kwekkeboom D, Perren A. Neuroendocrine gastro-enteropancreatic tumors: ESMO Clinical Practice Guidelines for diagnosis, treatment and follow-up. Ann Oncol 2012;23 Suppl 7:vii124-vii130.

(120) Winter JM, Narang AK, Mansfield AS et al. Resectable pancreatic small cell carcinoma. Rare Tumors 2011;3(1):e5.

(121) Heim S, Mitelman F. Cancer Cytogenetics: Chromosomal and Molecular Genetic Aberrations of Tumor Cells. Third Edition ed. Wiley-Blackwell; 2009.

(122) Shi C, Klimstra DS. Pancreatic neuroendocrine tumors: pathologic and molecular characteristics. Semin Diagn Pathol 2014;31(6):498-511.

(123) Thakker RV, Newey PJ, Walls GV et al. Clinical practice guidelines for multiple endocrine neoplasia type 1 (MEN1). J Clin Endocrinol Metab 2012;97(9):2990-3011.

(124) Maher ER, Yates JR, Harries R et al. Clinical features and natural history of von HippelLindau disease. Q J Med 1990;77(283):1151-1163.

(125) Mukhopadhyay B, Sahdev A, Monson JP, Besser GM, Reznek RH, Chew SL. Pancreatic lesions in von Hippel-Lindau disease. Clin Endocrinol (Oxf) 2002;57(5):603-608.

(126) Feldkamp MM, Gutmann DH, Guha A. Neurofibromatosis type 1: piecing the puzzle together. Can J Neurol Sci 1998;25(3):181-191.

(127) Crino PB, Nathanson KL, Henske EP. The tuberous sclerosis complex. N Engl J Med 2006;355(13):1345-1356.

(128) Capurso G, Festa S, Valente R et al. Molecular pathology and genetics of pancreatic endocrine tumours. J Mol Endocrinol 2012;49(1):R37-R50.

(129) Jiao Y, Shi C, Edil BH et al. DAXX/ATRX, MEN1, and mTOR pathway genes are frequently altered in pancreatic neuroendocrine tumors. Science 2011;331(6021):11991203. 
(130) Marinoni I, Kurrer AS, Vassella E et al. Loss of DAXX and ATRX are associated with chromosome instability and reduced survival of patients with pancreatic neuroendocrine tumors. Gastroenterology 2014;146(2):453-460.

(131) LoPiccolo J, Blumenthal GM, Bernstein WB, Dennis PA. Targeting the PI3K/Akt/mTOR pathway: effective combinations and clinical considerations. Drug Resist Updat 2008;11(1-2):32-50.

(132) Fjallskog ML, Lejonklou MH, Oberg KE, Eriksson BK, Janson ET. Expression of molecular targets for tyrosine kinase receptor antagonists in malignant endocrine pancreatic tumors. Clin Cancer Res 2003;9(4):1469-1473.

(133) Missiaglia E, Dalai I, Barbi S et al. Pancreatic endocrine tumors: expression profiling evidences a role for AKT-mTOR pathway. J Clin Oncol 2010;28(2):245-255.

(134) Yachida S, Vakiani E, White CM et al. Small cell and large cell neuroendocrine carcinomas of the pancreas are genetically similar and distinct from well-differentiated pancreatic neuroendocrine tumors. Am J Surg Pathol 2012;36(2):173-184.

(135) Oberg K. Neuroendocrine tumours in 2012: Insights into signalling pathways could individualize therapy. Nat Rev Endocrinol 2013;9(2):70-72.

(136) Ferreira BI, Alonso J, Carrillo J et al. Array CGH and gene-expression profiling reveals distinct genomic instability patterns associated with DNA repair and cell-cycle checkpoint pathways in Ewing's sarcoma. Oncogene 2008;27(14):2084-2090.

(137) Chial H. Cytogenetic Methods and Disease: Flow Cytometry, CGH, and FISH. Nature Eduation 2008;1(1):76.

(138) Wang Z, Gerstein M, Snyder M. RNA-Seq: a revolutionary tool for transcriptomics. Nat Rev Genet 2009;10(1):57-63.

(139) Mitelman F, Johansson B, Mertens F. Mitelman database of chromosome aberrations and gene fusion in cancer. http://cgap.nci.nih.gov/Chromosomes/Mitelman 2014.

(140) Bugalho MJ, Roque L, Sobrinho LG et al. Calcitonin-producing insulinoma: clinical, immunocytochemical and cytogenetical study. Clin Endocrinol (Oxf) 1994;41(2):257260.

(141) Long PP, Hruban RH, Lo R, Yeo CJ, Morsberger LA, Griffin CA. Chromosome analysis of nine endocrine neoplasms of the pancreas. Cancer Genet Cytogenet 1994;77(1):55-59.

(142) Scappaticci S, Brandi ML, Capra E, Cortinovis M, Maraschio P, Fraccaro M. Cytogenetics of multiple endocrine neoplasia syndrome. II. Chromosome abnormalities in an insulinoma and a glucagonoma from two subjects with MEN1. Cancer Genet Cytogenet 1992;63(1):17-21.

(143) Floridia G, Grilli G, Salvatore M et al. Chromosomal alterations detected by comparative genomic hybridization in nonfunctioning endocrine pancreatic tumors. Cancer Genet Cytogenet 2005;156(1):23-30.

(144) Speel EJ, Scheidweiler AF, Zhao J et al. Genetic evidence for early divergence of small functioning and nonfunctioning endocrine pancreatic tumors: gain of 9Q34 is an early event in insulinomas. Cancer Res 2001;61(13):5186-5192.

(145) Terris B, Bernheim A. [Comparative genomic hybridization (CGH): application to the study of neuroendocrine tumors]. Ann Pathol 1999;19(5 Suppl):S9-11.

(146) Zhao J, Moch H, Scheidweiler AF et al. Genomic imbalances in the progression of endocrine pancreatic tumors. Genes Chromosomes Cancer 2001;32(4):364-372.

(147) Maitra A, Hansel DE, Argani P et al. Global expression analysis of well-differentiated pancreatic endocrine neoplasms using oligonucleotide microarrays. Clin Cancer Res 2003;9(16 Pt 1):5988-5995.

(148) Couvelard A, Hu J, Steers G et al. Identification of potential therapeutic targets by geneexpression profiling in pancreatic endocrine tumors. Gastroenterology 2006;131(5):1597-1610.

(149) Capurso G, Lattimore S, Crnogorac-Jurcevic T et al. Gene expression profiles of progressive pancreatic endocrine tumours and their liver metastases reveal potential novel markers and therapeutic targets. Endocr Relat Cancer 2006;13(2):541-558. 
(150) Hansel DE, Rahman A, House M et al. Met proto-oncogene and insulin-like growth factor binding protein 3 overexpression correlates with metastatic ability in welldifferentiated pancreatic endocrine neoplasms. Clin Cancer Res 2004;10(18 Pt 1):6152-6158.

(151) Bloomston M, Durkin A, Yang I et al. Identification of molecular markers specific for pancreatic neuroendocrine tumors by genetic profiling of core biopsies. Ann Surg Oncol 2004;11(4):413-419.

(152) Duerr EM, Mizukami Y, Ng A et al. Defining molecular classifications and targets in gastroenteropancreatic neuroendocrine tumors through DNA microarray analysis. Endocr Relat Cancer 2008;15(1):243-256.

(153) Dilley WG, Kalyanaraman S, Verma S, Cobb JP, Laramie JM, Lairmore TC. Global gene expression in neuroendocrine tumors from patients with the MEN1 syndrome. Mol Cancer 2005;4(1):9.

(154) Carr JC, Boese EA, Spanheimer PM et al. Differentiation of small bowel and pancreatic neuroendocrine tumors by gene-expression profiling. Surgery 2012;152(6):998-1007.

(155) World Medical Association Declaration of Helsinki: ethical principles for medical research involving human subjects. JAMA 2013;310(20):2191-2194.

(156) Porembka MR, Hall BL, Hirbe M, Strasberg SM. Quantitative weighting of postoperative complications based on the accordion severity grading system: demonstration of potential impact using the american college of surgeons national surgical quality improvement program. J Am Coll Surg 2010;210(3):286-298.

(157) Bassi C, Dervenis C, Butturini G et al. Postoperative pancreatic fistula: an international study group (ISGPF) definition. Surgery 2005;138(1):8-13.

(158) Beasley TM, Schumacker RE. Multiple regression approach to analyzing contingency tables: Post hoc and planned comparison procedures. The Journal of Experimental Education 1995;64(1):79-93.

(159) Love MI, Huber W, Anders S. Moderated estimation of fold change and dispersion for RNA-Seq data with DESeq2. http://biorxiv.org/content/early/2014/05/27/002832 2014 May 27.

(160) Huang dW, Sherman BT, Lempicki RA. Systematic and integrative analysis of large gene lists using DAVID bioinformatics resources. Nat Protoc 2009;4(1):44-57.

(161) Andersson R, ndren-Sandberg A, Lundstedt C, Tranberg KG. Implantation metastases from gastrointestinal cancer after percutaneous puncture or biliary drainage. Eur J Surg 1996;162(7):551-554.

(162) Partelli S, Gaujoux S, Boninsegna L et al. Pattern and clinical predictors of lymph node involvement in nonfunctioning pancreatic neuroendocrine tumors (NF-PanNETs). JAMA Surg 2013;148(10):932-939.

(163) Capella C, Solcia E, Sobin LH, Arnold R. World Health Organization Classification of Tumours: Pathology and Genetics of Tumours of the Digestive System. Lyon: IARC Press; 2000.

(164) Parekh JR, Wang SC, Bergsland EK et al. Lymph Node Sampling Rates and Predictors of Nodal Metastasis in Pancreatic Neuroendocrine Tumor Resections: The UCSF Experience With 149 Patients. Pancreas 2012;41(6):840-844.

(165) Basturk O, Yang Z, Tang LH et al. The high-grade (WHO G3) pancreatic neuroendocrine tumor category is morphologically and biologically heterogenous and includes both well differentiated and poorly differentiated neoplasms. Am J Surg Pathol 2015;39(5):683-690.

(166) Micci F, Weimer J, Haugom L et al. Reverse painting of microdissected chromosome 19 markers in ovarian carcinoma identifies a complex rearrangement map. Genes Chromosomes Cancer 2009;48(2):184-193.

(167) Lundqvist M, Oberg K. In vitro culture of neuroendocrine tumors of the pancreas and gut. Acta Oncol 1989;28(3):335-339.

(168) Mergler S, Strauss O, Strowski $\mathrm{M}$ et al. Insulin-like growth factor-1 increases intracellular calcium concentration in human primary neuroendocrine pancreatic tumor cells and a pancreatic neuroendocrine tumor cell line (BON-1) via R-type Ca2+ 
channels and regulates chromogranin a secretion in BON-1 cells. Neuroendocrinology 2005;82(2):87-102.

(169) Wagner L, Templ E, Reining G et al. Culture of human insulinoma cells: development of a neuroendocrine tumor cell- and human pancreatic islet cell-specific monoclonal antibody. J Endocrinol 1998;156(3):469-476.

(170) Evers BM, Ishizuka J, Townsend CM, Jr., Thompson JC. The human carcinoid cell line, BON. A model system for the study of carcinoid tumors. Ann N Y Acad Sci 1994; 733:393-406.

(171) Pandis N, Bardi G, Heim S. Interrelationship between methodological choices and conceptual models in solid tumor cytogenetics. Cancer Genet Cytogenet 1994;76(2):77-84.

(172) Micci F, Skotheim RI, Haugom L et al. Array-CGH analysis of microdissected chromosome 19 markers in ovarian carcinoma identifies candidate target genes. Genes Chromosomes Cancer 2010;49(11):1046-1053.

(173) Xourafas D, Tavakkoli A, Clancy TE, Ashley SW. Distal pancreatic resection for neuroendocrine tumors: is laparoscopic really better than open? J Gastrointest Surg 2015;19(5):831-840.

(174) Kneuertz PJ, Patel SH, Chu CK et al. Laparoscopic distal pancreatectomy: trends and lessons learned through an 11-year experience. J Am Coll Surg 2012;215(2):167-176.

(175) Hackert T, Hinz U, Fritz $\mathrm{S}$ et al. Enucleation in pancreatic surgery: indications, technique, and outcome compared to standard pancreatic resections. Langenbecks Arch Surg 2011;396(8):1197-1203.

(176) Karaliotas C, Sgourakis G. Laparoscopic versus open enucleation for solitary insulinoma in the body and tail of the pancreas. J Gastrointest Surg 2009;13(10):1869.

(177) Crippa S, Bassi C, Salvia R, Falconi M, Butturini G, Pederzoli P. Enucleation of pancreatic neoplasms. Br J Surg 2007;94(10):1254-1259.

(178) Eom BW, Jang JY, Lee SE, Han HS, Yoon YS, Kim SW. Clinical outcomes compared between laparoscopic and open distal pancreatectomy. Surg Endosc 2008;22(5):13341338.

(179) Sanchez-Cabus S, Adam JP, Pittau G, Gelli M, Cunha AS. Laparoscopic left pancreatectomy: early results after 115 consecutive patients. Surg Endosc 2016.

(180) Ho CK, Kleeff J, Friess H, Buchler MW. Complications of pancreatic surgery. $H P B$ (Oxford) 2005;7(2):99-108.

(181) Pitt SC, Pitt HA, Baker MS et al. Small pancreatic and periampullary neuroendocrine tumors: resect or enucleate? J Gastrointest Surg 2009;13(9):1692-1698.

(182) Beger HG, Mayer B, Rau BM. Parenchyma-Sparing, Limited Pancreatic Head Resection for Benign Tumors and Low-Risk Periampullary Cancer-a Systematic Review. J Gastrointest Surg 2016;20(1):206-217.

(183) Jilesen AP, van Eijck CH, Busch OR, van Gulik TM, Gouma DJ, Nieveen van Dijkum EJ. Postoperative Outcomes of Enucleation and Standard Resections in Patients with a Pancreatic Neuroendocrine Tumor. World J Surg 2016;40(3):715-728.

(184) Bettini R, Partelli S, Boninsegna L et al. Tumor size correlates with malignancy in nonfunctioning pancreatic endocrine tumor. Surgery 2011;150(1):75-82.

(185) Kim MJ, Choi DW, Choi SH et al. Surgical strategies for non-functioning pancreatic neuroendocrine tumours. Br J Surg 2012;99(11):1562-1568.

(186) Zhang IY, Zhao J, Fernandez-Del CC et al. Operative Versus Nonoperative Management of Nonfunctioning Pancreatic Neuroendocrine Tumors. J Gastrointest Surg 2016;20(2):277-283.

(187) Strasberg SM, Drebin JA, Linehan D. Radical antegrade modular pancreatosplenectomy. Surgery 2003;133(5):521-527.

(188) Strasberg SM, Linehan DC, Hawkins WG. Radical antegrade modular pancreatosplenectomy procedure for adenocarcinoma of the body and tail of the pancreas: ability to obtain negative tangential margins. $J$ Am Coll Surg 2007;204(2):244-249. 
(189) Lee SH, Kang CM, Hwang HK, Choi SH, Lee WJ, Chi HS. Minimally invasive RAMPS in well-selected left-sided pancreatic cancer within Yonsei criteria: long-term (>median 3 years) oncologic outcomes. Surg Endosc 2014;28(10):2848-2855.

(190) Hashim YM, Trinkaus KM, Linehan DC et al. Regional lymphadenectomy is indicated in the surgical treatment of pancreatic neuroendocrine tumors (PNETs). Ann Surg 2014;259(2):197-203.

(191) Ricci C, Casadei R, Taffurelli G et al. The role of lymph node ratio in recurrence after curative surgery for pancreatic endocrine tumours. Pancreatology 2013;13(6):589-593.

(192) Krampitz GW, Norton JA, Poultsides GA, Visser BC, Sun L, Jensen RT. Lymph nodes and survival in pancreatic neuroendocrine tumors. Arch Surg 2012;147(9):820-827.

(193) Curran T, Pockaj BA, Gray RJ, Halfdanarson TR, Wasif N. Importance of Lymph Node Involvement in Pancreatic Neuroendocrine Tumors: Impact on Survival and Implications for Surgical Resection. J Gastrointest Surg 2014.

(194) Pomianowska E, Gladhaug IP, Grzyb K et al. Survival following resection of pancreatic endocrine tumors: importance of R-status and the WHO and TNM classification systems. Scand J Gastroenterol 2010;45(7-8):971-979.

(195) Tomassetti P, Campana D, Piscitelli L et al. Endocrine pancreatic tumors: factors correlated with survival. Ann Oncol 2005;16(11):1806-1810.

(196) Toste PA, Kadera BE, Tatishchev SF et al. Nonfunctional pancreatic neuroendocrine tumors $<2 \mathrm{~cm}$ on preoperative imaging are associated with a low incidence of nodal metastasis and an excellent overall survival. J Gastrointest Surg 2013;17(12):21052113.

(197) Ferrone CR. Lymphadenectomy for pancreatic neuroendocrine tumors: is that the relevant debate? Ann Surg 2014;259(2):213-214.

(198) Stabile BE. When pNETs bust out of their shells. Arch Surg 2012;147(9):828.

(199) Yoo YJ, Yang SJ, Hwang HK, Kang CM, Kim H, Lee WJ. Overestimated Oncologic Significance of Lymph Node Metastasis in G1 Nonfunctioning Neuroendocrine Tumor in the Left Side of the Pancreas. Medicine (Baltimore) 2015;94(36):e1404.

(200) Falconi M, Zerbi A, Crippa S et al. Parenchyma-preserving resections for small nonfunctioning pancreatic endocrine tumors. Ann Surg Oncol 2010;17(6):1621-1627.

(201) Fernandez-Cruz L, Cosa R, Blanco L, Levi S, Lopez-Boado MA, Navarro S. Curative laparoscopic resection for pancreatic neoplasms: a critical analysis from a single institution. J Gastrointest Surg 2007;11(12):1607-1621.

(202) Fox AM, Pitzul K, Bhojani F et al. Comparison of outcomes and costs between laparoscopic distal pancreatectomy and open resection at a single center. Surg Endosc 2012;26(5):1220-1230.

(203) Butturini G, Partelli S, Crippa S et al. Perioperative and long-term results after left pancreatectomy: a single-institution, non-randomized, comparative study between open and laparoscopic approach. Surg Endosc 2011;25(9):2871-2878.

(204) Singh N, Lo CY, Chan WF. Laparoscopic enucleation of a nonfunctioning neuroendocrine tumor at the head of the pancreas. JSLS 2006;10(2):259-262.

(205) Kim SC, Song KB, Jung YS et al. Short-term clinical outcomes for 100 consecutive cases of laparoscopic pylorus-preserving pancreatoduodenectomy: improvement with surgical experience. Surg Endosc 2012.

(206) Haugvik SP, Rosok BI, Waage A, Mathisen O, Edwin B. Single-incision versus conventional laparoscopic distal pancreatectomy: a single-institution case-control study. Langenbecks Arch Surg 2013;398(8):1091-1096.

(207) Drymousis P, Raptis DA, Spalding D et al. Laparoscopic versus open pancreas resection for pancreatic neuroendocrine tumours: a systematic review and metaanalysis. HPB (Oxford) 2014;16(5):397-406.

(208) ClinicalTrials.gov Registry. https://clinicaltrials gov 2016.

(209) Haugvik SP, Gaujoux S, Rosok B, Gladhaug IP, Dousset B, Edwin B. Laparoscopic versus open pancreas resection for neuroendocrine tumours: need for evaluation of oncological outcomes. HPB (Oxford) 2014;16(9):871. 
(210) Fratini G, Giudici F, Bellucci F, Batignani G, Tonelli F. Feasibility of Mesenteric Vein Reconstruction with PTFE Prosthesis for Non Functioning Endocrine Pancreatic Tumor Surgery. Pancreatic Dis Ther 2012;2(1).

(211) Ouaissi M, Hubert C, Verhelst $\mathrm{R}$ et al. Vascular reconstruction during pancreatoduodenectomy for ductal adenocarcinoma of the pancreas improves resectability but does not achieve cure. World J Surg 2010;34(11):2648-2661.

(212) Abu HM, McPhail MJ, Zeidan BA, Jones CE, Johnson CD, Pearce NW. Aggressive multi-visceral pancreatic resections for locally advanced neuroendocrine tumours. Is it worth it? JOP 2009;10(3):276-279.

(213) Panzuto F, Boninsegna L, Fazio $\mathrm{N}$ et al. Metastatic and locally advanced pancreatic endocrine carcinomas: analysis of factors associated with disease progression. J Clin Oncol 2011;29(17):2372-2377.

(214) Winter JM, Brennan MF, Tang LH et al. Survival after resection of pancreatic adenocarcinoma: results from a single institution over three decades. Ann Surg Oncol 2012;19(1):169-175.

(215) Nagenthiraja K, Ewertz M, Engholm G, Storm HH. Incidence and mortality of pancreatic cancer in the Nordic countries 1971-2000. Acta Oncol 2007;46(8):10641069.

(216) Shaib YH, Davila JA, El-Serag HB. The epidemiology of pancreatic cancer in the United States: changes below the surface. Aliment Pharmacol Ther 2006;24(1):87-94.

(217) Sharpe SM, In H, Winchester DJ, Talamonti MS, Baker MS. Surgical resection provides an overall survival benefit for patients with small pancreatic neuroendocrine tumors. J Gastrointest Surg 2015;19(1):117-123.

(218) Crippa S, Partelli S, Bassi C et al. Long-term outcomes and prognostic factors in neuroendocrine carcinomas of the pancreas: Morphology matters. Surgery 2015.

(219) Shiba S, Morizane C, Hiraoka N et al. Pancreatic neuroendocrine tumors: A singlecenter 20-year experience with 100 patients. Pancreatology 2015.

(220) Frilling A, Modlin IM, Kidd M et al. Recommendations for management of patients with neuroendocrine liver metastases. Lancet Oncol 2014;15(1):e8-21.

(221) Partelli S, Inama M, Rinke A et al. Long-Term Outcomes of Surgical Management of Pancreatic Neuroendocrine Tumors with Synchronous Liver Metastases. Neuroendocrinology 2015;102(1-2):68-76.

(222) Heetfeld M, Chougnet CN, Olsen IH et al. Characteristics and treatment of patients with G3 gastroenteropancreatic neuroendocrine neoplasms. Endocr Relat Cancer 2015;22(4):657-664.

(223) Khan MS, Luong TV, Watkins J, Toumpanakis C, Caplin ME, Meyer T. A comparison of Ki-67 and mitotic count as prognostic markers for metastatic pancreatic and midgut neuroendocrine neoplasms. Br J Cancer 2013;108(9):1838-1845.

(224) La RS, Sessa F. High-grade poorly differentiated neuroendocrine carcinomas of the gastroenteropancreatic system: from morphology to proliferation and back. Endocr Pathol 2014;25(2):193-198.

(225) Velayoudom-Cephise FL, Duvillard P, Foucan L et al. Are G3 ENETS neuroendocrine neoplasms heterogeneous? Endocr Relat Cancer 2013;20(5):649-657.

(226) Shi C, Gonzalez RS, Zhao Z et al. Liver metastases of small intestine neuroendocrine tumors: Ki-67 heterogeneity and World Health Organization grade discordance with primary tumors. Am J Clin Pathol 2015;143(3):398-404.

(227) Zen Y, Heaton N. Elevated Ki-67 labeling index in 'synchronous liver metastases' of well differentiated enteropancreatic neuroendocrine tumor. Pathol Int 2013;63(11):532-538.

(228) Janson ET, Sorbye H, Welin S et al. Nordic guidelines 2014 for diagnosis and treatment of gastroenteropancreatic neuroendocrine neoplasms. Acta Oncol 2014;53(10):12841297.

(229) Labori KJ, Haugvik SP, Line PD. Letter to the editor: Multidisciplinary management of pancreatic neuroendocrine tumors - the importance of an aggressive surgical approach. Scand J Gastroenterol 2016;51(3):383-384. 
(230) Kulke MH, Siu LL, Tepper JE et al. Future directions in the treatment of neuroendocrine tumors: consensus report of the National Cancer Institute Neuroendocrine Tumor clinical trials planning meeting. J Clin Oncol 2011;29(7):934943.

(231) Terris B, Meddeb M, Marchio A et al. Comparative genomic hybridization analysis of sporadic neuroendocrine tumors of the digestive system. Genes Chromosomes Cancer 1998;22(1):50-56.

(232) Hechtman JF, Beasley MB, Kinoshita Y, Ko HM, Hao K, Burstein DE. Promyelocytic leukemia zinc finger and histone H1.5 differentially stain low- and high-grade pulmonary neuroendocrine tumors: a pilot immunohistochemical study. Hum Pathol 2013;44(7):1400-1405.

(233) Bowen SL, Bloor BK, Leigh IM, Waseem A. Adducin expression in cutaneous and oral lesions: alpha- and beta-adducin transcripts down-regulate with keratinocyte differentiation in stratified epithelia. J Pathol 2003;201(1):119-126.

(234) Moon RT, Kohn AD, De Ferrari GV, Kaykas A. WNT and beta-catenin signalling: diseases and therapies. Nat Rev Genet 2004;5(9):691-701.

(235) Kaldis P, Pagano M. Wnt signaling in mitosis. Dev Cell 2009;17(6):749-750.

(236) Voloshanenko O, Erdmann G, Dubash TD et al. Wnt secretion is required to maintain high levels of Wnt activity in colon cancer cells. Nat Commun 2013;4:2610.

(237) Fox SA, Richards AK, Kusumah I et al. Expression profile and function of Wnt signaling mechanisms in malignant mesothelioma cells. Biochem Biophys Res Commun 2013;440(1):82-87.

(238) Nakashima N, Liu D, Huang CL, Ueno M, Zhang X, Yokomise H. Wnt3 gene expression promotes tumor progression in non-small cell lung cancer. Lung Cancer 2012;76(2):228-234.

(239) Zhang J, Li Y, Liu Q, Lu W, Bu G. Wnt signaling activation and mammary gland hyperplasia in MMTV-LRP6 transgenic mice: implication for breast cancer tumorigenesis. Oncogene 2010;29(4):539-549.

(240) Zhang KS, Zhou Q, Wang YF, Liang LJ. Inhibition of Wnt signaling induces cell apoptosis and suppresses cell proliferation in cholangiocarcinoma cells. Oncol Rep 2013;30(3):1430-1438.

(241) Rulifson IC, Karnik SK, Heiser PW et al. Wnt signaling regulates pancreatic beta cell proliferation. Proc Natl Acad Sci U S A 2007;104(15):6247-6252.

(242) Kim JT, Li J, Jang ER et al. Deregulation of Wnt/beta-catenin signaling through genetic or epigenetic alterations in human neuroendocrine tumors. Carcinogenesis 2013;34(5):953-961.

(243) Grande E, Capdevila J, Barriuso J, Anton-Aparicio L, Castellano D. Gastroenteropancreatic neuroendocrine tumor cancer stem cells: do they exist? Cancer Metastasis Rev 2012;31(1-2):47-53.

(244) Chen G, A J, Wang M et al. Menin promotes the Wnt signaling pathway in pancreatic endocrine cells. Mol Cancer Res 2008;6(12):1894-1907.

(245) Kim JT, Liu C, Zaytseva YY, Weiss HL, Townsend CM, Jr., Evers BM. Neurotensin, a novel target of Wnt/beta-catenin pathway, promotes growth of neuroendocrine tumor cells. Int J Cancer 2015;136(6):1475-1481.

(246) Ansell SM. Wnt-erizing mantle cell lymphoma. Blood 2008;112(13):4783-4784.

(247) Ekeblad S, Lejonklou MH, Stalberg P, Skogseid B. Prognostic relevance of survivin in pancreatic endocrine tumors. World J Surg 2012;36(6):1411-1418.

(248) Grabowski P, Griss S, Arnold CN et al. Nuclear survivin is a powerful novel prognostic marker in gastroenteropancreatic neuroendocrine tumor disease. Neuroendocrinology 2005;81(1):1-9. 


\section{Appendix}

Surgical treatment algorithm for sporadic PNENs

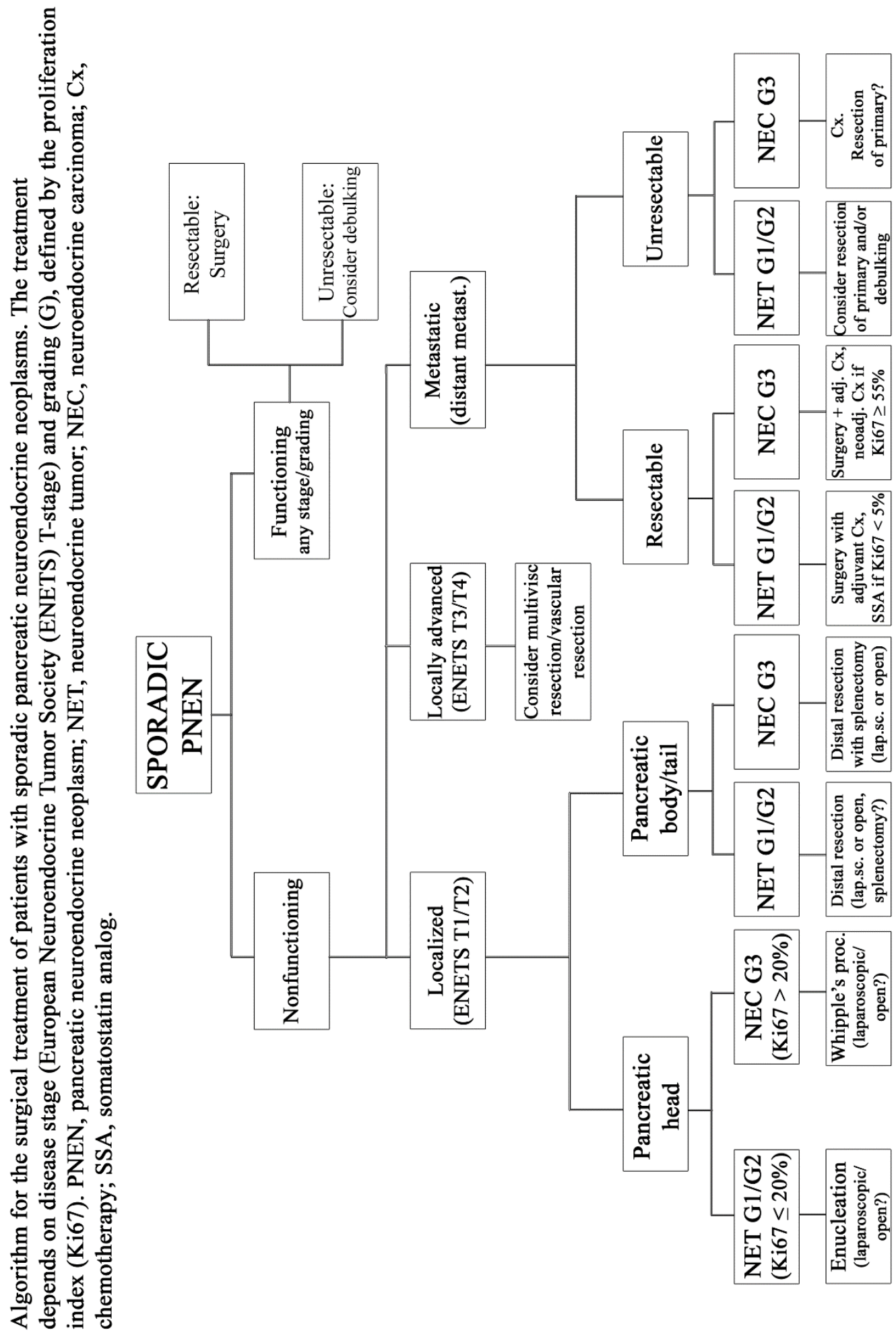


Surgical treatment algorithm for PNECs

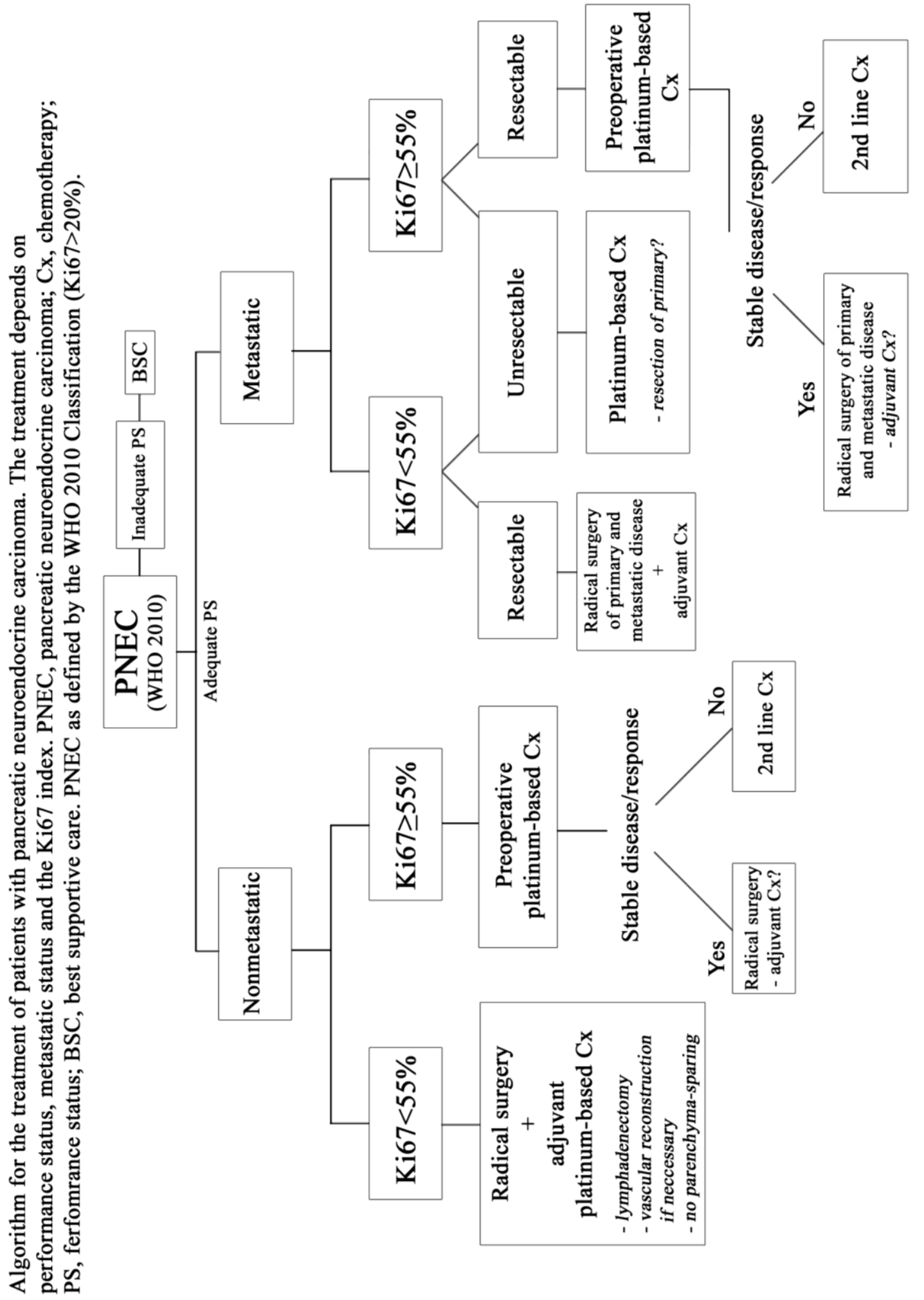

